

$\operatorname{tin} 9$.

Wisth the Canciliment of

the wither

Rev. Rephurin Sngethar th I. P. D. 

11

Nenominee Guide

seawen"

Catholic Procizer Pook. 



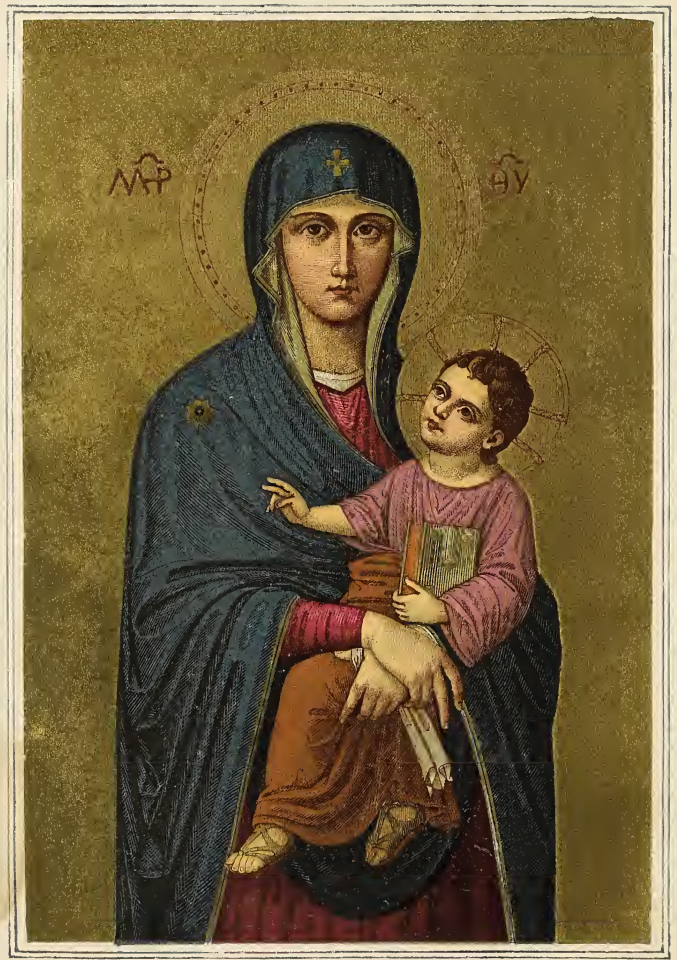

KÄZ MARIE, WÄKIACHSEME JESUS KEKETOTAMOWEACH! 


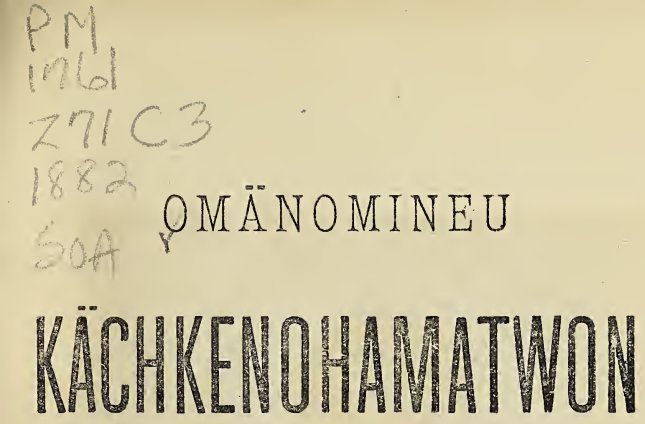

\section{KESEKOCH.}

\section{KATOLIK}

\section{ANAMIHÄU MASENACHIGON.}

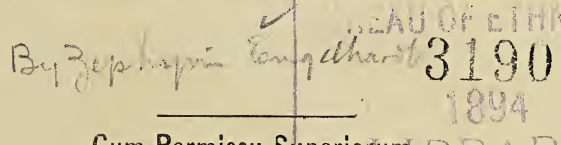

Cum Permissu Superiorum, $? R A R Y$

ST. LOLIS, MO.

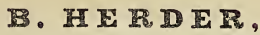

1882. 
Käz Mishäel,

Kaiäz Asäniwon,

Kit Anonik,

Mäz Hawätok,

Asau Kanaweheon,

O Mänominewon,

Kanaweheach,

Keketotamaweach!

Käz Otäh Jesus,

Sawänemeach !

Käz Marie, Wäkiachseon llawätok, .

Keketotamaweach!

Käz Joseph, kaies pemehazin Jesus,

Kanaweheach ! 


\section{TÄPANENON NEMAT OMÄNOMEU:}

Jom masenachekon äiawis as masenächnatek Omänomineu. Kon ne päz Omänominewenesenon. Ene tä kinau mächkan kotänos as kes kewazetaion. Å. wichkichtawon net äs asaton. Eneko nesik mänätamechkemakach pos äs pachtamach, pos äsetaiach, omos masenachegone weskewa anamihawak, asau aweiach. Kespin ene esetaiach ios nekoch masenachekone naiäma, kinau onanekose kinau näwonau Täpänemenach, kaies sikihinach. Ene wä iom masenachekon Kächkenohamatwon Kesekoch as asachzekatäk, asau wechtamach achpäniu asau esetuiach äkoiach. Anamihätawena, ene tä achpäniu neu asä onanekosiach kesekoch.

"Wämänominewenesit," Mächkotächkoniäu. 



\section{Pronunciation of Letters.}

The letters are to be pronounced as in the Otchipwe books.

Pronounce a like the letter a in father,

\begin{tabular}{|c|c|c|c|}
\hline $\mathrm{e}$ & 6. & 6 & a "s made, \\
\hline $\mathrm{g}$ & 66 & 66 & $\mathrm{~g}$ " go, \\
\hline $\mathrm{h}$ & 66 & 66 & h " hat, \\
\hline $\mathrm{i}$ & 66 & "s & i " wind, \\
\hline $\mathrm{k}$ & "6 & 6 & k " keep, \\
\hline $\mathrm{m}$ & 66 & "6 & m " Mary, \\
\hline $\mathrm{n}$ & 16 & 66 & n " no, \\
\hline o & 66 & 6 & o " note, \\
\hline $\mathrm{P}$ & "6 & 16 & $\mathrm{p}$ " priest, \\
\hline 8 & 66 & 6 & s "6 sit, \\
\hline $\mathrm{t}$ & 66 & "6 & $\mathrm{t}$ " table, \\
\hline$u$ & 66 & "letters & oo" moon, \\
\hline w & 66 & "letter & w " wet, \\
\hline $\mathbf{z}$ & 66 & $\begin{array}{l}\text { "letters } \\
\text { man }\end{array}$ & $\begin{array}{l}\text { ts or the ger- } \\
\text { in } z u \text {, }\end{array}$ \\
\hline$\ddot{a}$ & 16 & $\begin{array}{l}\text { "letter } \\
\text { had, }\end{array}$ & a in mad or \\
\hline $\mathrm{ch}$ & 66 & $\begin{array}{c}\text { "Germa } \\
\text { ach. }\end{array}$ & an ch in ich, \\
\hline
\end{tabular}

Other letters or sounds are only used in foreign words and proper names. 


\section{KÄZ MESACHKATAWÄN.}

Kaies ani nishino metatach nekot ene peponakesit änamibat, naskat mesachkatawäu, tachnäno wätächzechnächkin mesachkatawäu kesegaton. Kat katäu mesachkatawäu tä, kätin käz maze esetau. Tachnin päpochkin otächzechnin ene mäz mesachkatawän, niu eno metatach tachno- kon mesachkatawäwak änamihatoa, os pos kotakihisitoa o päzetonon io. Pächkichkesekach enechpe waiäpechne ene käz mesachkatawän. Pachk kesekach tä $\theta-$ nechpe paionechne. Pos mesachkatawäu tä ächtachne kesekach ächpez neu käz mesachkatawäu. Anamihäkesekachkin tä kon pos mesachkatawänon. .Kon pos metchenon metchemechsä apächtawechne, (Joseph kesekach), misik tä Anamihäwachtik kesekach. Pos metchin metchemechsä : 1) kes ani Anamihäkesekachkin nisho konakach; 2) Ispinigon kesekach miniu; nekoz nekotäs nesik pos metchin metchemechsä echpo naiawachkikin mit- 
chichsi. Manikesekach pos metchin metcemächsä, ene nesik nish Manikesekachkin. Ene tä ki pos mesachkatawäm tä äsis aiakoamemena Mäz Mächkotäcbkoniäu. 


\section{KÄZ MESACHKATTAWANN.}

- Pepon. Pächzichlesegot pos mesachiatawäm nanas Pachk kesekach.

1883 - 7 Nanäpin keso, — 25 Wanäu keso.

1884 - 27 Namäpin keso, - 13 Sopomach keso.

1885-18 Namäpin keso, — 5 Sopomach keso.

1886 - 10 Wanäu keso, - 25 Soponach keso.

1887 - 23 Namäpin keso, - 10 Sopomach keso.

1888 - 15 Namäpin keso, - 1 Somopach keso.

1889 - 6 Wanäu keso, - 21 Sopomuch keso.

1890 - 19 Namäpin keso, - 6 Sopomach keso.

1891 - 11 Namäpin keso, - 29 Wanäu keso.

1892 - 2 Wanäu koso, - 17 Sopomach kesc。

1893 - 15 Namäpin keso, - 2 Sopomach keso.

1894 - 7 Namäpin keso, - 25 Wanäu keso.

$1895-27$ Namapin keso, - 14 Sopomach keso。

1896 - 19 Namäpin keso, - 5 Sopomach keso.

1897 - 4 Wanäu keso, - 18 Sopomach kego。

1898 - 28 Namäpin keso, - 10 Sopomach keso.

1899 - 15 Namäpin keso, - 2 Sopomach keso.

1900 - 28 Namäpin keso, - 15 Sopomach keso.

1901 - 20 Namäpin keso, — 7 Sopomach koвo.

1902 - 12 Namäpin keso, - 30 Wanäu keso.

1903 - 25 Namäpin keso, - 12 Sopomach keso.

1904 - 17 Namäpin keso, - 3 Sopomach keso.

1905 - 8 Wanäu keso, - 23 Sopomach keso.

$1906-28$ Namäpin keso, - 15 Sopomach keso. 


\section{MESACHKATAWÅU KESEGATON, os pos mesachratawä, kat os pos metche motche- mechsa.}

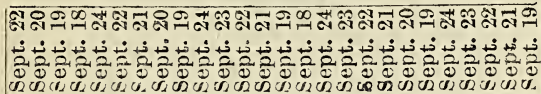

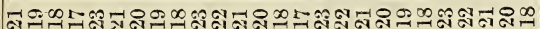

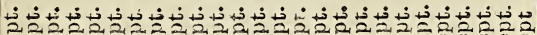

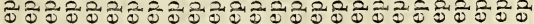

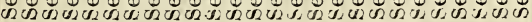

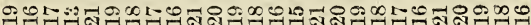

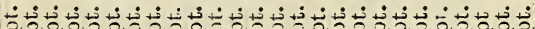

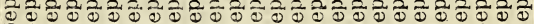

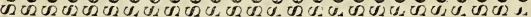

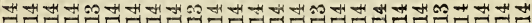

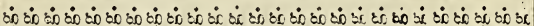

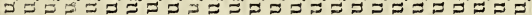

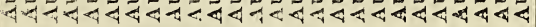

ब्न की

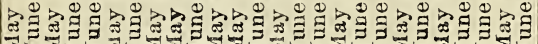

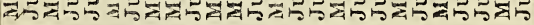

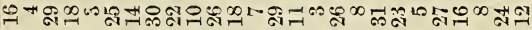

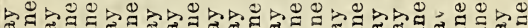

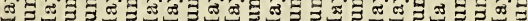

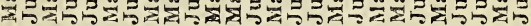

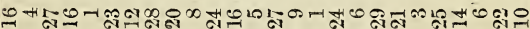

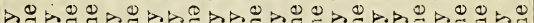

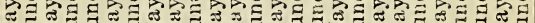

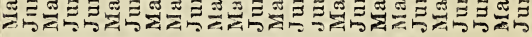

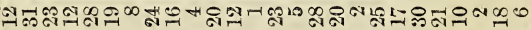

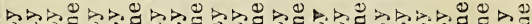

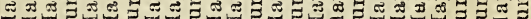

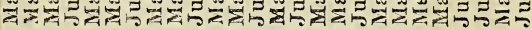

\% సै

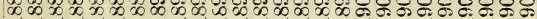
开 


\section{MESACHKATAWÄU KESEGATON,}

\section{os pos mesachiata.wä, kat os pos metche metche. mechsa.}

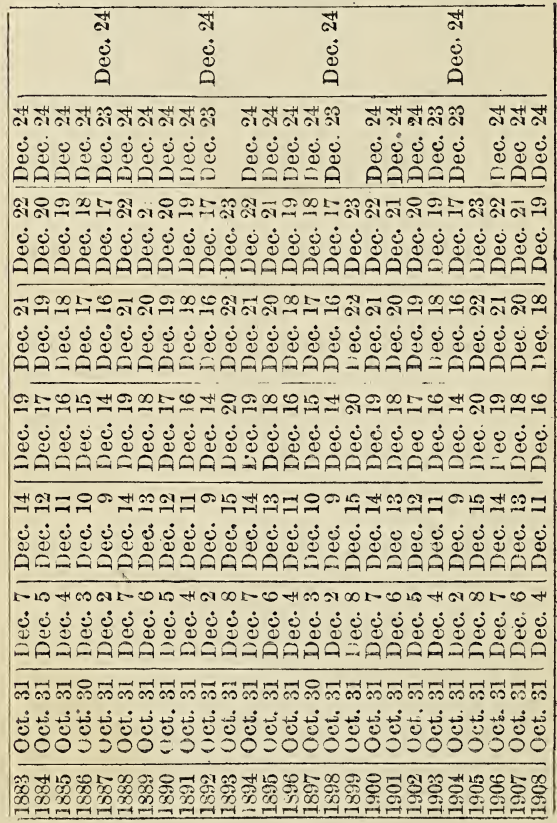




\section{KÅZ KESEGATON.}

1. Nipatepä Anamihäkesegot. (December 25). Otächzechnin tachnäno nishino metatach nianon ene tachnokonakesit Hawätok kesonochsä.

2. Anamächkatoan kesegot. (January, 1). Otächzechnin tachnäno waiäpakemechzin Hawätok keso.

3. Okemau kesegot. (January, 6). Otächzechnin nekotoasita tachnokonakesit Hawätok keso.

4. Asäniwon as kes pis anamächkakot käz Marie. (March 25). Nishino metatach nianon ene tachnokonakesit Wanäu keso.

5. Enechpe Jesus kesekoch äsiat. Kes ani Pachk kesegot niu metatach nokon.

6. As Pemochne kesegot, enechpo mänazechtoch Jesus Weau Käz Ostiwine äpit. Nekotoasita metatach nish ene kesegoton kes ani Pachk kesegot.

7. Enechpe käz Marie kesekoch äsiat. (August, 15). Otächzechnin tachnäno metatach nianon ene tachnokonakesit Manomäch keso. 
8. Käz Mishän kesegot, kaies känawehazin Omänominewon. (September, 29). Nishino metatach sakäu ene Anawipiama* kot keso.

9. Mawau käz Achpechtänctakositoa kesegot. (Norember, 1). Waiäpakemechzin Kipatin keso.

10. Päzeton kon o kes kekeochtatesenon käz Marie. (December, 8). Otaichrechnin tachnäno shuasik tachnokonakesit IIawätok kesonochsä. 
Poponon.

¿ichpo Jesus kesokoch äsiat.

1883

1884

1835

1886

1887

1888

1889

1890

1891

1892

1893

1894

1895

1896

1897

1898

1899

1900

1901

1902

1903

1904

1905

1906

1907

1908
3 Pachkoan keso 22

14

3 Atähämin "

19 Pachkoan “"

10

30

15

$7 \quad 66$

26

11 "6

3

$66 \quad 66$

6

"6

"

“

"

"

23

14

27

19

11

24

16

8

21

12

1 Atähämin

24 Pichkoan

9
28

Pemochner kesegot.

24 Pachkoan keso

12 Aiähümin “

4 " "6

24 66 6

9 "6 6

31 Pachkoan "6

20 Atiibämin "

5

28 Pachkoan "6

16 Atähämin "6

1

24 Pachkoan "

13 Atähämin "

4

17

9 66 6

1

14

6

29 Pachionn "

11 Atähämin "

2 "

22 "

14

30 Pachkoan "

18 Atühümin " 


\section{ANAMIHANON.}

- O Wichswone Wäwochneme misik Wäkiseme misik Waieskesit Hawätok. Amen.

\section{Nochnenau Kesekoch Äpeon.}

Nochnenau kesekoch äpeō. Nas tä kat käz achpechıänechton ki wichswon. Nas 1ä kat pimakat kit okcmawin. Änänechtamon nas tä kat esekemakot tepa kesekoch misik ios achkihe

Meseach iochpe as kesekach ne pachkishigonemenau ächtanächkin kesegaton. Ponikitätaweach miniu kaies nächkehekäon, äsis ponikitätawakichtoa kaies nächkihiamächtoa. Pon miniu ishiaseach kakiztipänichtoane. Miakonamoweach tä mätä. Amen.

\section{Kit Anomächkon Marie.}

Kit anomächkon Marie, moskinanikoian sawänechzekon; Täpänechzekät ki witchiak. Ki käz achpechtänetakosim ächtasitoa metämochsok, käz achpechtänetakoseu miniu kaies kikäskawat, Jesus. 


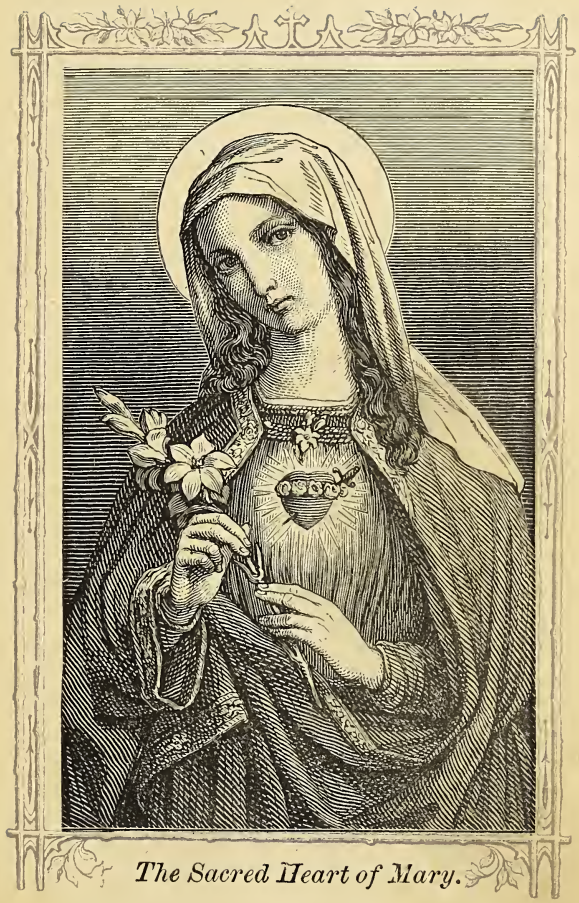



Käz Marie, Wäkiachseme Hawätok, keketotamoweach äs päzetaiach, iochpe, misik tepachikone nepäiach. Amen.

Käz Achpechtänetakosit Wäwochneme.

Käz achıpectänetakosit Wäwochneme, misik Wäkiseme, misik Waieskesit Hawätok. Jachpez käz achpechtänetakosit käios, iochpe miniu, achpäniu miniu, kakikächkamik. Amen.

\section{Ne Papamächtawau.}

Ne papamächtawau Hawätok Wäwochneme, mesas käkoch nähechtok, kaies kesechtok kesik achkäu miniu. Ne papamächton miniu Okison nekoneneu aiawenit, Jesus Christ, Täpänemenach. Waieskesinit Hawätokon kes mamazetawehek; Manion kaiekachkoenit kes ochtateseu. Kes kotakechtau as mäk ohemawenit -Ponze Pilatan, kes sachsakachkohau anamihäwachtiko, kes nipoa, kes nekuahau miniu. A namachkiach kes isiu, as näno konakach kes iach apiskau. Kosehoch kes isiu, mesächsin ot aninianech Hawätokon Wäwochneme mesas käkoch nähechtok. Ene 
tä au iochpiat, asau pis tepachkonazin pematesinit, näpenik miniu.

Ne papamächtawau Waieskesit Hawätok. Ne papamächton käz Katolik Anamihäwigamik, käz achpichtisitoa o witochkatoanonwau, päzetonon kisehekatäwon, iachapiskak weau, kakik pematesin. Amen.

\section{Asäniu ot Anamihan.}

$V$. Täpänemenach ot asänimon kos wochtamowäu Marion.

R. Eno tä Waieskesinit Hawätokon as kes mamazetawehekot Jesus. Kit anomächkon Marie etc.

v. Marie kes hewa: Ne täpänemik Täpänemenach.

R. Ene häion, one au esekoon. - Kit anomächkon Marie etc.

V. Kus metchemechseweheso Hawätok Okison.

R. As kos wez tanachkiamenach. - Kit anomächkon etc.

V. Käz Marie, Hawätok Wäkiachsoon, keketotamoweach.

R. Asau meseach Jesus kaiesis wawechtamuweach. 
IIawätok Täpänemenach, ki pakichsenemekem asau wieskeseach, äsis otätchiakiach; ki kes pis anonau kit asänim, asau wechtach as katäu pis mamazetawihäsit Kikis Jesus; ene tä Kikis kes kotakehau, kes nänau miniu anamihäwachtiko, nenach io; ene wä pakichsänemekäon asau mächno apiskaiach. Amen.

\section{Paketchekon Anamilian.}

Ne wechtamoau Hawätok mesas käkoch nähechtok; käz Marie kakik kaickachkoit; käz Michael, kaiäz asäniwit; käz Joan Baptist; käz Pien misik tä Paul; mawau kesekoch ächtanäsitoa ne wechtamowawak, misiktä kenach nochne, ke wechtamon: as kes päzetaion, as kes päz inänechtamon, as kes päz ean, as kes maze eset:iion miniu; nenach net äsikin, nenach net äsikin, nenach käz net äsikin. Ene wä mamiachtomakoa, käz Marie, kakik kaiekachkoit; käz Michael, kaiäz asäniwit; käz Joan Baptist; käz Pion misik tä Paul; mawau kesekoch ächtanäsitoa, asạu keketotamowitoa, misik kenach nochne keketotamowenach. Amon. 


\section{Aninichswon.}

Hawätok Nochne, wächzetau net aninichsim as kes nächkehenon, as ke päzetaion. Wächzetau ki käz achpechtänetakosim, ene wä sechkatamon mawau ne maze esekenon; kon käkoch net äsis sechkatanon, äsis sechkatamon ne maze esekin; kenıch wä sechkatamon, ächpez waieskeseon, ächpez miniu tapanekoseon. Sawänemena, kisehamawena, Jesus o wichswone io kisehamawena. Ne kes kesänechton kon misik asau päzetaion. Wotochkawena, sawänemena, kon misik nenau esekenon; ene au enänemeon ene kakik au esekeon. Amen.

\section{Onamichtamowin.}

Hawätok Täpänemeach, mawau kaiesis kächkenohamaweach äsis anamihaiarh, ki kes etämepach, ene mawau wä papamächtamach. Amen.

\section{Pakichsänichtamowin.}

Hawätok 'Täpänemeach, wächzetau kätin net achpänemenau keau, Jesus o kotakechton io, asau wetochkaweach, kwai- 
achk asau isis pemateseach omos achkihe, . kakik miniu kesekoch asau onanekoseach. Amen.

\section{Tapanitwon.}

Hawätok Täpänemeach, wächzetau ki käz achpechtänetakosim, wächzetau miniu ki tapanekem; ene wä tapanekäon opäsik, mawau tä äsikimakach. Ne tapanonawak miniu mawau pämatesitoa, kenach wä tapanakichtoa; ächpez tapanisiach ene ächpez tapanakichtoa. Amen.

Nekotoasita tachnäno anino, pos küchkenach, pos onamichtach miniu änamihat.

1. Asi Mäz Hawätok nekoneneu.

2. Hawätok kwaiachk tepachkonikäu; maiächno pematesinit mächno totawäu, mäze pematesinit tä kotakehäu, mämik enis, 'kes ani pematesinit achkihe.

3. Näniu Nekoneneu Hawätok, Wäwochneme, misik Wälkiseme, misik Waieskesit Hawätok.

4. Hawätok Okison, eno kä Hawätok Wäkiseme, Jesus, kes pis mamazetaweheso, kes kotakechtau, kes nipoa miniu 
anamihäwachtiko, kenach io, kakik pematesin kesekoch asau menenach.

5. Kitätchiakonawak kakik au pematesewak, kon nekotoch pos o näpinowawon.

6. Kon weiak pos o wichkichtonon käkoch asau mächno esetot, asau mächno esekit miniu, konomächtchiu asau menekot Hawätokon sawänächzekon; ene nesik Hawätok o sawänechzckon wichkichtawach asau mächno esekeach, kesekoch miniu asau isiach kes ani pemateseach achkihe.

Ene kätau kächkenach, kätau onamichtach miniu änamihat, asau wichkichtok kesekoch asau isiat, kespin konau mächno esekit.

Metatach Mäz Hawätok Ot Aiakoamech nesenon.

1. Nekoneneu Mäz Hawätok mesas käkoch kaies kesechtok; nekoneneu kinau menazehau, änekochkitähäion kinau tapanau.

2. Kon nasop Hawätok kinau wawechnanon. 
3. Anamihäkesegot kinau menazechton.

4. Kochne kikia miniu kinau menazehawak, kinis asau pemateseon omos achkihe.

5. Pon weiak näsin.

6. Pon maze wekemewänon.

7. Pon kimotenon.

8. Pon kinochkenon, asau pachtamot weiak.

9. Pon mochnawenamowin kez pemateseu waiekemazin.

10. Pon mochnawenamowin kez pemateseu käkoch ächtanach.

Nekotoasita 'Katolik Anamihäwigamik Ot Aiakoamechnesenon.

1. Kinau menazechton Käz Kesegaton.

2. Kinau anamiham as mäk Mäz Anamihan Anamihäkesekachkin Käz Kesekachkin miniu.

3. Wäwänin kinâu paketchekäm tachnäno nekot pepon; kinau otachpenom miniu Käz. Eucharistiwin tachnäno wätechzechnäkin Pachk.

4. Kinau mesachkatawäm niu metatach kesegaton konomächtchiu asau otächzech- 
ne Pachk. Pon metche metchemechsä Anamihäwachtik kesekach.

5. Käkoch kesekechtawon, kinau anamihäu paketenekäm, äsitatoa änamihatoa.

6. Pon wekemin osam kächze änawemat; pon nasewänon, weiak anamihäu nepawit, echpe wätächzechnächkin as kinuatich.

Käz Achpechtänetakosinon.

1. Sikahapuanitwon. 2. Sochkitähäskatwon. 3. Paketchekon. 4. Käz Eucharistiwin. 5. Anamihäu Peme. 6. Mächkotächkoniäwiwin. 7. Anamihäu Wikichtwon.

Nohekon käz Päzetonon.

1. Maminatesin. 2. Positchiwin. 3. Päsikoatesiwin. 4. Kishiawänichtoan. 5. Nipatesin. 6. Nächkosin. 7. Tatakatchichkiwin.

Nekotoasita Päzetonon wä-näihkehe Waieskesit Hawätok.

1. Osam weiak as achpänemit Hawätokon äsis kisäwatesinit.

2. Osam mänawaz weiak as achpänemot Hawätokon ano kisewatesinit. 
3. Osam weiak as kinochtach onamichtoan, ano as kächkenach as onamimakach.

4. Weiak as kakawänemąin wez mamazetawon, Hawätok o sawänechzekon as kakawänemazin.

5. Weiak wächzetau päzetone as tase pematesit.

6. Weiak kon nekotoch as aninichsit nanas asau nepäk.

Niu enon Päzetonon kesekoch äs aio-ohohemakaton.

1. Weiak wächzetau nänazin woz pematesewon.

2. Sodoma o päzeton. (Nekot Päshikoatesiwin).

3. Weiak maze totawazin kätemakesinit, saiekawenit, kaiewesinit miniu.

4. Woiak päz tepahamowazin kaies anochkit.

Sakäu kaiäneswak o päzetoniwawon äs wechtek.

1. Weiak as kekomazin asau päzetonit.

2. Weiak as aiakoamemazin asau päzetonit. 
3. As mininechtamä weials as päzetot.

4. Weiak as kakachsächta nanas asau päzetonit.

5. As minuazeme weiak as päzetot.

6. As kiane weiak kaics päzetazin.

7. As wetochko weiak as päzetot.

8. Kat as aianächne weiak, kespin kat säsewaho päzetot.

9. As natamo, as mächno echpänone tä miniu, weiak päzetazin.

Näniu käz mächno esetonon.

1. Anamihan. 2, Mesachkatawän. 3. Sawänechzikan.

Nohekon mächno esetonon maikatimakakin mamach päzetonon.

1. Tapachnenichswon mikatimakat maminatesin.

2. Keshiatesin mikatimakat positchiwin.

3. Penesewin mikatimakat päsikoatesin.

4. Tapanitwon mikatimakat kishiawänechzekan.

5. Tipamänimowin mikatimakat nepatesin. 
6. Nawanou esekin mikatimakat nächkosin.

7. Nänepewin mikatimakat tatakatchichwin.

Nohekon mächno esetonon äsis mächno echpänane wez pematesewon äs oweawenit.

1. As achsamichtoa pachkatätoa.

2. As minahichtoa paichkapakitoa.

3. As pichtikanichtoa as weke kaiänesewak.

4. As akihichtoa kätemakesitoa.

5. As papam wapamichtoa waiesakesitoa.

6. As tipahochtoa tächkonichtoa (asau anich-kotakihichtoa).

7. As pakitänimichtoa näpikoa. Nokekon mächno esetonon äsis mächno echpünane weiak äsis otätchiakit.

1. Wäwänin as anineme päzetot.

2. As kächkenohamochtoa kon käkoch kächkinachkoa.

3. As mächno kakichkimichtoa kon käkoch kächkinachkoa au esetatoa.

4. As nahäkazimichtoa kaiäz koskänehtachkoa. 
5. As mächno otachpenamochtoa maiächno totawikitoa.

6. As ponikitätochoa kaies nächkihikitoa.

7. As keketotamochtoa pämatesitoa näpikoa tä miniu.

Niu enon echpe anihimakach mamazetau o pematesin.

1. Nepän. 2. Tepachkonikäwin. 3, Anamachkiach. 4. Kesekoch.

Shuasik Sawänechtakosenon.

1. Sawänechtakosewak kätemakesitoa otätchiakoa, akiwak kä wätachpinichkoa okemawin kesekoch.

2. Sawänechtakosewak maiächno esetähätoa, akiwak kä achkäu au tipänichtachkoa.

3. Sawänechtakosewak koskänichtachkoa as kes maze esetatoa, akiwak au mininechtachkoa.

4. Sawänechtakosewak pachkatätoa, paichkepakitoa tä minin kwaiachk pematesin, akiwak au täpinachkoa.

5. Sawänechtakosewak sawänechzekätoa, akiwak au wänimiichtoa. 
6. Sawänechtakosewak painitahätoa, akiwak kä Hawätokon au näwatoa.

7. Sawänechtakosewak nawanen isikitoa akiwak kä Hawätok onitchionechson au inichtoa.

8. Sawänechtakosewak kwaiachk pä。 matesitoa kotakihichtoa Anamihan io, akiwak kä wätachpinichtoa okemawin kesekoch.

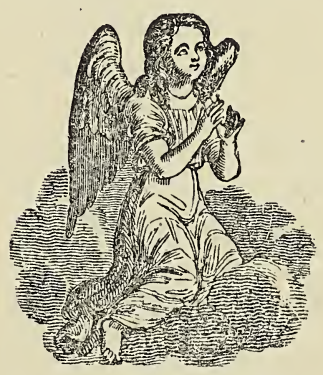


26

\section{MIP ANAMIIIAN.}

Kakichkotakan asau kächkenamä, au esechzekä echpe wänechkin.

Kit äsis akuamemin, papik neu asau mächkawänemot Hawätok koskoscanin. Wäneanin anamihäwatikonikenon, eta tä miniu :

Hawätok Nochne, ki mamiawamin, as kes kanaweheon tepä.

Papik tä onenon posächkakänon tä miniu, ene tä asau otchichtchikoanetaion änanametot anamihäwachtik, kespin tanat. Zenopomin anamihäwachtik asau mächkawänemot Jesus kaiesis kotakechtok anamihäwachtiko, kenach io. Wäwänin tä anamihanon tachnäno mip.

Paketenamowin Hawätok keau tä miniu ki tätchiakon. Mawau tä miniu eneko au enänechtamon, au ean, au esetaion miniu cnech kesekach paketenamowin Hawätok. Eita tä :

Hawätok Nochne, ki pakitänamon neau ne tätchiak miniu. Änänechtamon ene au esekeon kisähächkoa. Sawänemena achpäniu asau anochkitonon! 
Kespin neanekotochkin osam wätametaiąnin, mänawaz nokoz anamihanon。 Pakuana tä kinach kächkenanon anum anamihanon, eno kä: "Nochnenau lieselioch aipeon;" "Kit anomächkon Marie;" "Ne papamächtawau Hawätok Wäwochneme;" $O$. namichtamowin; Pakichsänichtamowin; Tapanitwon; Aninichswon miniu, anamihan masenachigone atäwon. Eta tä mawau neu anum anamihanon tachnäno mip. Kespin tä kat otametaion mawau neu anum ächkichnächkin anamihanon wechtach.

Mip Anamitianon.

\% O Wichswone Wäwochneme misik Wäkisemo misik Waieskesit Hawätok. Amen.

"Nochnenau kesekoch äpeon" elc "Kit anomächkon, Marie," etc "Ne papamächtawau Hawätok Wäwochneme," etc

"Käz achpechtänetakosit Wäwochneme," etc.

Mamiawamatwon.

Hawätok mesas käkoch nähechtawon, kinach mawau neu iachpez täpänemeach, 
ki mamiawamekem as kes kanaweheach tepä. Kakik sawänemeach, kakik kinach papamächtakäm au äz pemateseach. Amen Aninichswon.

Ponikitätaweach kaiesis päzetaiach; kätin ne sechkatämenau ne maze esekenon; kon misik nєnach katäu nähe isiki ninawon; misik sawänemeach nähe sawä. nechzekäion; kinuawin saiechkaseach kon kotäu asau totaweach. Amen.

\section{Paketänamatwon.}

Ene wä paketänichsiach äsis oweaweach äsis otätchiakeach, Täpänemeach! Kakik tepänechtach au esekeach, enekoch tä miniu au kitämakeseach; kon pos ne wichkichtoninawon os pos tepänichsiach. Nekoneneu as pakichsenemekäion, asau kwaiachk esekeach as mäk pemateseach, kes nepäiach miniu kesckoch asau piatä. weheach.

\section{Onamichtamowin.}

Hawätok Täpänemeach, mawau kaiesis kächkenohamaweach äsis anamihaiach, ki kes etämepach, ene mawau wä papamäch. tamach. 


\section{Pakichsänichtamowin.}

Hawätok Täpänemeach, wächzetau kätin net achpäniminau keau, Jesus o kotakechton io, asau wetochkaweach, kwaiachk asau isis pemateseach omos achkihe, kakik miniu kesekoch asau onanekoseach.

\section{Tapanitwon.}

Hawätok Täpänemeach, wächzetau ki käz achpechtänetakosim, wächzetau miniu ki tapanekem; ene wä tapanekäon opäsik, mawau tä äsikimakach. Ne tapanonawak miniu mawau pämatesitoa, kinach tapanakichtoa; ächpez tapanisiach, ene ächpez tapanakichtoa. Amen.

\section{Misik mip anamihanon.}

Net asänimenawak kit anonekowau kit okemawenau Jesus asau papawitchiweach ; papawitchiwea, kakik mächkamawea, äsis kächkenohamakäiach; kakik kinuachkon saiechkaseamä, pon käkoch asau totaweamä.

Käz Joseph, kinach mämik mamazetau achkäu känàwechtamon : ki kes onapamik 
Jesus, ki sochkänemekem asau keketotamoweach. Ki tapanik Jesus as kes kanawehat achkibe, kinau papamächtak asau keketotamoweach.

Käz Mishäel, kinach kaiäz asäniweon, kinach kaies nochkehat maze hawätok, wetochkaweach, nena nap asau nochkehake saiekaseamä.

Kinach waiez owichswonemenach, keketotamoweach, kaiesis anamihaion achkihe, nena nap tä asau isis anamihaion kakik tä miniu kesekoch asau wekemenach kes nepäion achkihe.

Maiächno apeäk kesekoch, ki tapanekowau mesas käkoch kaies kesechtok naiäwäk; keketotamoweach tatepa asau mächno apeach kesekoch.

Josus sawäremeach! Jesus sawänemeach! Jesus sawänemeach!

Käz Marie, wäkiachsemekäion, keketotamoweach!

Käz Joseph, keketotamoweach!

Mawau neu kesekoch ächtanachkiek, nazekapawetochkon Hawätok, asau keketotamoweach. Amen. 


\section{Asäniu Anamihan (Angelus).}

Ene tä wechtach Asäniu anamihan.

Tachnäno mip, nawachkik, nächka minii, nänino kotiwächnin nenawät, asau otchichtchikoaneta, ene tä asau etamä ene anamihan "Asäniu Anamihan", as wechtek Ene tä ene wä anamihatoa enechpe änamihatoa, asau menazichtokoa Jesus as kes mamazetawihäsit, konau tä asau wäwänin asau inatoa Täpänechzekänit, as kes mamazetawihäsinit, asau inänihazin kitätchiakonawon.

Memorare.

Jachpez achpichtänichtakwot ene anamihan.

Mächkawänechtach, käz Marie, kaiekachkoeon, nähe sawänechzekäon, kon nekotoch kes isis nochtanon, asau kes anichtawat weiak kaies mamiachtomächkin asau keketotamowat. Kaiekachkoeon mamawau naiätamateseon wäkiachsemenon, nena nap, ano käz päzetaion äsıs achpänemeon keau, ki pis nazenesemin, ki pis nasächkon as koskänechtamon as kes päzetaion, $\mathrm{ki}$ pis otchichtchikoanapéton. 
Jesus wäkiachsemot, pon paketach äsis mamiach tomenon; sawänochtawenach miniu papamächtawenach. Amen.

Anamihan ätamä asau konomächtchiu mitchisi.

Achpäniu anamihanon asau konomächtchiu mitchichsion, wechtach tä nekotono: "Nochnenau kesekoch äpeon" nekotono miniu: "Kit anomächkon Marie" nekotono miniu: "Käz achpechtänetakosit Wüwochneme." Ene tä wechtach: ,Mesas käkoch kaies kesechtawon, mächno anochkitakäon äsis otätchiakiach, äsis oweaweach miniu. Amen."“

Anamihan ätamä kaies ani mitchichsikin. Achpäniu anamihanon kaies ani mitchichsion, asau wäwänin enat Hawätok as kes achsamä, wechtach tä nekotono "Nochnenau kesekoch äpeon," nekotono miniu: "Kit anomächkon Marie," misiktä : "Käz achpechtänetakosit Wäwochneme." Ene tä eta: "Mesas käkoch kaies kesechtawon, ki mamiawamekem kaiesis achsameach. Sochkitähäskaweamä achpäniu asau papamächtakäion. Amen." 


\section{NÄCHKAN ANAMIHAN.}

Kakichkotakan asau kächkenamä, au esetach, kätau nepachkin.

Kit äsis aiakoamemim, wäwänin asau anamihaion tachnäno nächka kätau nepaionin. Wäwänin esen Hawätok as kes kanawehe iochpe as kesekach. Nanakatawänichsinon, kaiesis päz ean, kaiesis päzetaion miniu iochpe as kesekach. Jachpez änekochkitäbäon aninichsinon, as kes nächkehat Kochne iachpez käsiatesit kesekoch äpit. Moskawänechtach, kon nekotoch asau päz esekeon. Paketenamo-. win Täpänechzekät keau kitätchiakon miniu, asau kanawehe iochpe as wanitipächkach. Mamiachtomin kaiekachkoit käz Marie, mawau miniu kesekoch äpitoa, asau keketotamochkoa.

Achpäniu penesenon, mämik kaisäkonaienon kätau nepaionin miniu. Mächkawänemin Hawätok, ki zenopamik, pon zenapachtach mätä, pon pächta miniu. Papik paketach maze inänechtamoan maze monawänıchtamoan miniu. Otachpenach 
anamihawapo miniu anamihäwachtikonikenon ene tä au isis osechsinon.

\section{Nächkan Anamihanon.}

* O Wichswone Wäwochneme misik Wäkiseme misik Waieskesit Hawätok. Amen.

Hawätok mesas käkoch nähechtawon, kinach mawau iachpez täpänemeach; ki mamiawamekem as kes kanaweheach iochpe as kesekach. Kakik sawänemeach, kakik kinau papamächtakäm au äz pema-teseach.

Kat ki nanakatawänechtä nawaz kaiesis päz esekeach iochpe as kesekach; as kes maze inänechtamach, as kes maze kikitiach, as kes maze esetaiach miniu, kon as kes esetaiach miniu wächzetau.

Ene tä omos asau nanakatawänichsi nemewach, koneu tä ene pos etan:

Hawätok Nochne, wächzetau net aninichsim as kes nächkehenon, as kes päzetaion. Wächzetan ki käz achpechtänetakosim, ene wä sechkatamon mawau ne maze esekin; kon käkoch net äsis sechka- 
tamon, äsis sechkatamon ne maze esekin; kina wä sechkatamon, ächpez wieskeseon, . ächpez miniu tapanekäon. Sawänemena, kisehamawena, Jesus o wichswon io kisebamawena. $\mathrm{Ni}$ kes kesänechton kon misik asau katäu päzetaion. Wetochkawena, sawänemena, kon misik nenau esekenon; au inänemeon ene kakik au esekeon.

Hawätok Nochnenau nähe sawänechze」 käion, kitämakänemeach, kon misik anoz ni kes maze esekin iochpe as kesekach; misik tä naskat sawänemeach, ponikitätaweamä kaiesis päzetaiach; moskawänechtameheach wäwänin asau aninichsiach, wäwänin achpäniu asau kanawehesiach nanas asau anich pemateseach achkihe. Täpänemeach Hawätok, Kikis Jesus, o. wichswon io ki pakichsenemin asau kitämakänemeach, asau paketamaweach mawau ne päzetonenawon.

Ponikitätaweach kaiesis päzetaiach; kätin ne sechkatämenau ne maze esekinawon; kon misik nenau esekenon; misik tä sawänemeach, nähe sawänechzekäon 
kinuawin saiechkaseach àsau konomächtchiu käkoch totaweach.

Hawätok Nochne, sochkitähäskawenach, kakik asau kwaiachk inänechtamon. Ki pakctänamon neau, nitätchiak, ne pematesin. Au inänimeon eneko au pemateseon sawänemeach asau kwaiachk pemateseon; inänimeon tä iochpe as wanitipächka asau nepäion, sawänemeach asau mächno nepäion.

Mesas kakoch kaies kesechtawon, ki papamächton net achpänimim keau, ki tapanin, ki katäu papamächton; ne sechkaton ne maze esekin; wetochkaweach asau nipoachkaion, ispämiach kesekoch miniu asau ision kes nepäion achkihe.

$\mathrm{Ki}$ pis nazenesimikäm, käz Marie, Hawätuk Wäkiachseme, pon paketach äsis mamiachtomikäon as kitämakeseach; kakik kanaweheach käkoch mäte kätau otächnikoiachkin. Kaiekachkoeon käz achpechtänetakoseon, iachpez tapanekäon, mamawau naikaneseon, Jesus Wäkiachsemot, kinach kakik kaiketotamoweach, keketotamoweach asau ponikitätaweach 
Jesus kaiesis päzetaiach. Keketotamoweach, ishiaseach asit Kikis Jesus.

Kit anomächkon, wäneseon Marie, mawau kez metämochsok kit anehawak; Hawätok Okison kit okiachsemau, konau tä ki kes kekachkowin. Käz Marie, Wäkiachsemekäon, keketotamoweach. Marie kaiekachkoeon iachpez käz achpechtänetakoseon! Kinach kon nekotoch kaies kikäskamon ene päzeton, ki kes ochıtatesitoa mawau pämatesitoa, keketotamoweach, pakichsenemin Jesus, Kikis Hawätokewit, asau mesit o sawänechzekon, asau tapanok Hawätok opäsik mawau käkoch, asau tapanakoa miniu pematesewak äsis tapanision. Amen.

Marie nähe sawänechzckäon kinach mamachkatänichtakwot. Kinach kon käkoch kikes weachsechkakon päzeton. Kanawänemena kanawänemena. Amen. "Nochnenau kesekoch äpeon." "Kit anomächkon Marie."

"Ne papamächtawau Hawätok." "Käz achpechtänetakosit Wäwochneme." "Memorare" ene kä: Mächkawäneshtach.

Naszeau Hawätok sawänemazin kisichnasoane ïpenit. 
38

\section{ANAMJHÅMIGUK.}

Kakichkotıkan asau kächkenamä, au esetach wäwänin asau akimichtoa anamihämiguk.

Kit änänemekonau Täpänechzekät asau menazehake kaiekachkoit käz Marie. Hawätok Okison ot okiachsemau .käz Maric miniu kinach kit okiachsemonau. Wäkiachsä kinau isis tapanau, wänitchionechsä kinau echpänonik. Kächkenowapomin onoch iachpez kaiäz achpechtänetakosit kaiekackkoit; kaies esekit esekenon. Pakichsenemin asau kanawehe, mämik natotamowin penetehäu, tapachnenichswon, waieskewach miniu nepän. Wätächnekoianin maze änänechtamon, papik nazenesemin Marie, kinau wetochkok asau nochkehat maze Hawätok. Asau lseketotamoch tä achpäniu, akemin anamihämignk wawepoz, wäwänin miniu akemin.

Kaiekachkoit käz Marie tepenau kes kächkenohamowäu käz Dominikon iom anamihämiguk tä äs wechzekasowak. Ene tä eno kaiäz achpetesit Mächkotächkoniäu as kes papam kakichkotakät, mesewäu asau akematoa anamihämiguk. Jachpez 


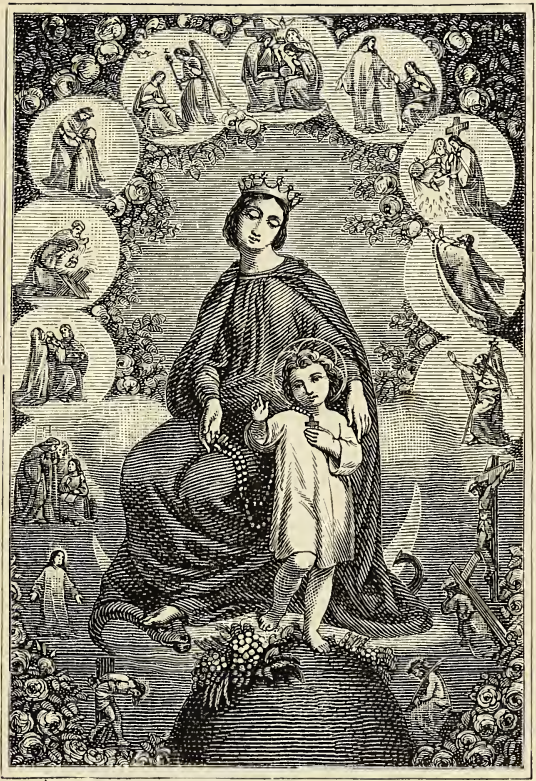

\section{The Lady of the Rosary.}



wepoz änamihatoa änekochkoa achkäu kes akemäwak anamihämiguk, ene tä mitchinịu äsichziketoa. Kätin manäwak kaies wichkichtoasitoa kakik pematesin kesekoch as kes menazehatoa wäwänin kaiekachkoenit käz Marion, as kez akimatoa miniu wawepoz anamihämigon.

Ene kätau esetaion kinach, menazchazin käz Marie tapasin miniu änekochkitähäion, akemin miniu anamihämiguk iachpez wawepoz; echpech tä akimatoa anamihämiguk, mächkawänechtach kaiesis mamazetawehäsit Täpänemenach Hawätok Okison; kaies pis inänihazin pämatesinit; kaiesis ochtatesit käz kitämakesine pisächkiwikamiko; kaies kotakechtok kinach io anamihäwachtiko; kaiesis nepäk miniu. Mächkawänechtach miniu kaiesis iachpematesit näno konakach ; kaiesis isiat kesekoch; kaiesis otachpenazin miniu' käz Okiän ot okemawine kesekoch. Ene au esechzekäon wäwänin tä kinau akemawak anamihämiguk. Sawänechtakoseu änamihat, tachnäno kesekach äkimazin anamihämigon! Au mininechtamihäu käz .Ma- 
rion, au käz sawänemäu kisichnasoane kotakechtonik, au wichkichtoaso mäse sawänechzekon, kätau io mächno anochkitawazin Hawätokon, ene tä nekotoch kesekoch asau isiat. Mämik akiko pämatesitoa kon nänachkoa masenachigon as mäk Mäz Anamihan, tachnäno miniu nächkachkin as anamiha.

Anamihämiguk.

I O Wichswone Wäwochneme misik Wäkiseme misik Waieskesit Hawätok. Amen.

"Nepapamächtawau Hawätok Wäwochneme" etc.

"Käz achpechtänetakosit Wäwochneme" etc.

"Nochnenau kesekoch äpeon".

Ene tä nänino: "Kit anomächkon Marie"

1. Maikit opäsik mäse kwaiachk onamichtamon.

2. Maikit opäsik mäse sochka pakichsänichtamon.

3. Maikit opäsik mäse kakaiänit tapanitwon.

"Käz achpechtänetakosit Wäwochneme" etc. 
I. Onanikinichtamon.

1. Kenach kaiekachkoeon, kaies kikäakawat, Waieskesit Hawätok io.

2. Kenach kaiekachkoeon, kaies kikäskawat, as isitawat Elisabeth.

3. Kenâch kaiekachkoeon, kaies onitchionechsemot.

4. Kenach kaiekachkoeon, kaies paketenot anamihäwigamiko.

5. Kenach kaiekachkoeon, kaies machkawat anamihäwigamiko.

II. Kotakechton.

1. Kaies kipotchisit mächki.

2. Kaies sächsewaho.

3. Kaies pacbsächkipene minichsakächsin.

4. Kaies pemenekonazin ot anamihäwachtikomon. tiko.

5. Kaies sasakachkoho anamihäwach-

III. Achpechtänetämä.

1. Kaies iachpematesit.

2. Kaics isiat keseknch.

3. Kaies pis enäschazin Waieskesit Ha* wätokon.

4. Kaies pis otachpene kesekoch.

5. Kaies okemakiwihe kesekoch. 


\section{ANAMIHAN MIUHKICHNON.}

Saiochkanamihatoa mesewäu änekochkoa achkäu kanawechtawak käz Marie ot isowenäkakächsä, Scapular tä, äs wechtek, konopoz miniu michkichnon, asau menazehatoa kaiekachkoenit käz Marion. Achkano Hawätok Okiän kes wapachtämäu weau nekot kaies käz mächno esekinit inäniwon, Simon Stock kaies äne, ene tä as kes menazin michkichnon. Kätin iachpez käz achpichtänichtakwot ene kaiäz isowenäkakächsä, käz Marie ot isowenäkakächsä. Kanawechto wäwänin, achpä• niu miniu posächka, konau tä mächno esekin, menazeher kaiekachkoit käz Marie tapasin tä miniu, wenach tä wänitchionchsä kinau isis kanawehek, achpäniu tä miniu kinach keketotamak, mämik tä echpe nepäion. Kinach käz mächno esetam kikition tachnäno kesekach nianon eno "Nochnenau kesekoch äpeon," nianon eno "Kit anomächkon Marie," asau menazehat kaiekachkoit käz Marie. 


\section{ANAMIHAN WAWEPOZ ÄTAMÄ.}

Käz Marie, Wäkiachseme Hawätok, anamihätawena, as maze esekeon! Mamiachtomin Kikis Jesus, asau mesit mächno esetähäwin, tapachnenichswon waieske. wach tä miniu tapanitwon, änekochkitähäion asau tapanak Hawätok, kinach nap tä asau käz tapanenon. Achpäniu ki katäu kächkenowapomin. Ki kes käz penetähäm, sawänemena, nenach nap achpäniu asau penetähäion. Hawätok kit okisemenau, achpäniu tä ki kes tapachnenichsim; keketotamawena tä miniu nerach nap asau tapachnenichsion. Ki käz tapanau Kikis Jesus, wetochkawena miniu nenach nap asau käz tapanak. Kit äsis pakichsenemin, kina Wäkiasemenon, achpäniu asau kanaweheon kat nekotoch päsikoaz asau esekeon. Mämik tä kanawehena keketotamawena tä miniu katäu anich pemateseon, asaủ mächno nepäion, ene tä kakik asau wez tanachkiamenon kesekoch. Amen.

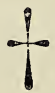




\section{KOTAKIHISWON MIHIKON.}

\section{Kakichkotakan.}

Jachpez weskewach asau mächkawänechtamäch neanekotochkin, kaiesis kotakechtok 'Täpänechzekät, as kes pis sikihazin kitätchiakonawon. Käz achpichtisitoa tä iochpe kesekoch äpitoa käz wawepoz kes nanakatawänechtawak Jesus o kotakechton, konau tä kes käz kitämakesewak Täpänechzekänit, kes nawemäwak miniu kaiesis kotakechtok. Ene äsechzekewak saiochkitähäskawatoa. Neanekotochkin nanakatawänechtawatoa Jesus o kotakechton, pemochnewak anamihäwachtik mihikon.

Kätin iachpez mächno esekeu änamihat, as mäk pemochnet anamihäwachtik mihikon. Naiächtamechne manäwak änamihatoa Sherusaleme kes isiwak, asau pemochnetoa kotakihiswon mihikon, kaies pemochnet tepenau Täjänechizekät, as kes pemenekonazin ot anamihäwachtikomon. Nätom kes pis nasächkawak Ponze Pilatan weke, kaies tepachkonik Jssus anamihä- 
wachtiko asau nepäk. Nàwenau tạ kes pis nasächkawak enoch wazeu kaies sachsakachkohäu Täpänechzekät, Kalvaria tä äs wechtek, konemau miniu Golgata. Kes pemochnewak tä anamihäwachtik mihikone, kes käz koskänechtomuk, kes nawemäwak miniu, as kes mächkawänechtachkoa: "Ene omos kaies kotakechtok Täpänechzekät; ene omos kaies sekechnin mawau o mächkom; ene omos kes nipoa anamihäwachtiko nenach io kakik iskotia asau akuachsemeon." Opäsik tä miniu kes tapanäwak Jesuson. Kes manäwak tä änamihatoa kon o kes wichkichtonowawon enis minikano asau isiatoa, kaies tase kotakechtok Mäz Hawätok Okison. Osam sanakat asau isiat, osam kinis ene minikan. Ene tä anèch änamihatoa kaies ioch osechtok anamihäwachtik mihikon mosenechtchigonon, anamihäwigamiko tä kes akotawak akiko mosenechtchigonon, ene tä enis anamihäwigamiko kes pemochnewak anamihäwachtik mihikon. Ene esekewảk iochpe mitchiniu. 
Saiochkitähäskatoa änekochkoa achkäu wawepoz pemochnewak kotakihiswon mihikon, konau tä nanakatawänechtomuk wäwänin kaiesis wechsakänechtach Jesus as kes pemenekonazin anamihäwachtikon. Ene pos esetach, kinach nap. Wawepoz pemochnenon kotakihiswon mihikon, opäsik tä kinau kächkenon kaiesis kotakechtok Täpänechzekät, asau inänimot kitätchiakonawon, opäsik miniu as tapanazin. Mämik kinau käz mächno esekin kotakihiswon mihikon echpe käz mesachkatawäwak änamihatoa konomäctchiu Pachk as otächzechne, misik tä Anamihäwachtik kesekachkin Anamihäkesekachkin miniu.

Aiom kaies mächno pemochnet anamihäwachtik mihikon wichkichtoaso Käz Kisehamatwon Sawänechzekon: Ene pos wichkichtoasenach wawepoz enoch Sawänechzekon kisichnasoane kotakechtokoa, Hawätok asau otachpenazin kesekoch.

Asau mächno pemochneon tä kotakihiswon mihikon, ene au esetaion. Pakichsenemin asau sawäneme, asau mächno pemochneon anamihäwachtik mihikon, ko- 
nau tä änekochkitähäon aninichsinon. Nas tä wichkichtoeon Miakonamatwon Sawänechzekon, asau sawänemazin Hawätok, asau sawänemazin miniu kisichnascrane äpenit, ene au änänechtamon enechpe. Nas tä Hawätok sawänemazin kinekehekok, ki nitchionechsok miniu, ene au anänechtamơn.

Nawenau tä kinau nasächkawak kotakihiswon mosenechtchigonon nanekot. Nätom kinau nämin mosenechtchigon, konau tä nanakatawänechtach Jesus o kotakechton. Nawenau tä kinau otchichtchikoanekopa wechtach tä miniu nekotono "Nochnenau", "Kit anomächkon Mari," "Käz achpechtänetakosit." Kes anamihaion tä, kinau nipitach, misiktä kaiänit mosenechtchigon kinau nasächkawau, ene tepach tä au esetaion misik, kaies esetaion nätom.

Ene au esetaion konomächtchiu akum metatach niu ene mosenechtchigonon nanekot. Aiatchkwot tä, wepoz anamihanon Käz Nochnenau änänechtamon asau wichkichtoaseon Kisehamatwon Sawänechze- 
kon. Käz weskewach asau eta "Nochne-

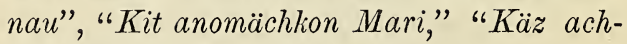
pechtänetakosit," nekotoasita eno.

Aiom wechtach masenachigon, mächno nanakatawänechtach, kaiesis maze totawit Jesus, pos eta miniu nekotono "Nochnenau," "Kit anomächkon Mari," "Käz xchpechtänetakosit," tachnäno* nasächkawazin nekot mosenechtchigon.

Kespin tä Mächkotächkoniäu kotakihiswon mihikon pemochnet, mawau anamihäwigamiko äpitoa pos pächtawäwak Mächkotächkoniäwon änamihazin, pos mächno nachkomäwak miniu, mosenechtchigonon tä monakach au korhketawak.

\section{Stabat Mater.}

Stabat Mater dolorosa

Juxta crucem lacrymosa,

Dum pendebat Filius.

\section{Anamihan.}

Jesus Täpänemeon, kätin ki tapanin, as kes käz kotakechtawon, as pis sawänemeon, ki kes nepäm asau meseon kakik pämatesin kesekoch. Nenach tä kon wä- 


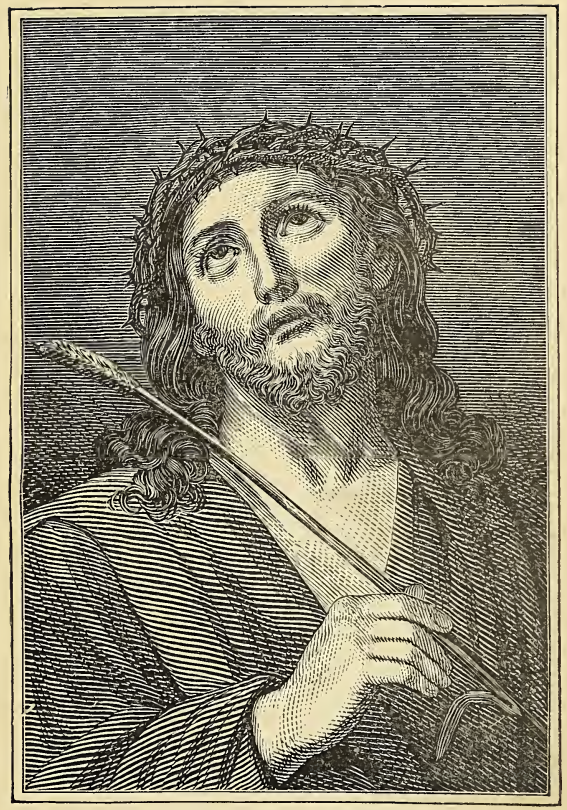

Behold the Man 

wänin ki tapanenänon, achpäniu ki mazetoton. Kätin ne sechkanèsim, net aninichsim; wäwänin iochpe ki katäu tapanin, ki katäu mächno anochkiton; wetochkawena, moskawänichtamihina. Amen.

Cujus animam gementem,

Contristatam, et dolentem,

Pertransivit gladius.

\section{I.}

Jesus kes tepachkonau asau nepäk.

Kit anamihätom, Jesus Krist, ki käz menazehekäm miniu.

As kes akuasimatoa pämatesitoa käz anamihäwachtiko as tachpänäon.

$\mathrm{Ne}$ nanakatawänechton omos, kaiesis echpänane Jesus. Kaies ani säsewahokä tä, kaies ani pasächkipene miniu minichsakächsion, tepachkonau Ponze Pilatan kes tepachkonik, asau nepäk anamihäwachtiko.

Jesus käshiateseon, nenach kä kaies maze echpänànenon, nenach kaies tepachkonønon as kes päz esekion. Ponikitäta- 


\section{0}

wena, Jesus Nochne, kon misik nenau esekenon. Opäsik ki katäu tapanin, sawänemena. Amen.

Nochnenau..... Kit anomächkon..... Käz achpechtänetakosit.....

Sawänemeach Täpänemeon * Sawänemeach.

Hawätok, kitäınakänemeach * Äs päzetaiach.

O quam tristis et afflicta

Fuit illa benedicta

Mater Unigeniti!

\section{II.}

Jesus otachpenäu ot anamihäwachtikomon.

Kit anamihätom...... As kes......

Ne nanakatawänechton omos, kaiesis kotakechtok Jesus, as kes otachpenazin ot anamihäwachtikomon. Mawau weau kes mächkoweu kes käz sächsewahau, ano tä kes otachpenäu o käz anamihäwachtikomon, kes pemenekonau, enis asau tachpänat ächpez tapanenach.

Jesus Täpänemeon, ne sechkaton mawau ne nächkosin, ne mazttähänon; wäwänin 
iochpe ne katau otachpenom mawau kotakechton, wesakesin, kitämakesin, mawau miniu käkoch sänakach, enekoch au meseon, nanas asau anich pemateseon. Amen.

Nochnenau...... Kit anomächkon...... IKäz achpechtänetakosit......

Sawänemeach, 'Гäpänemøon * Sawänemeach.

Hawätok kitämakänemeach * Äs päzetaiach.

Quae moerebat et dolebat,

Pia Mater, dum videbat

Nati poenas inclyti.

\section{III.}

Jesus papechzin nätom ot anamihäwachtikomon.

Kit anamihätom...... As kes.....

$\mathrm{Ne}$ nanakatawänechton omos, kaiesis anemesit $J$ esus as kes pemenckonazin, ene tä kes papechzin achkihe. Osam ni kes maze totawau, käz mäse mächki saiä kes sekechnin, ene tä kaies io anawechtok.

Jesus nähe sawänemeon, sawänemena, kakik asau kächkenawajomenon. As kes 
papechzenon, semakanechsok ki kes papahamekok asau nepäon; kenach tä kon pochz nekotono ki kes kikitinon. Enänemena, Jesus miniu nenach tepach asau sinawaneu esekeon, kespin maze echpänonekäon. Amen.

Nochnenau..... Kit anomächkon...... Käz achpechtänetakosit......

Sawänemeach...... Hawätok kitämakänemeach......

Quis est homo, qui non fleret,

Matrem Christi si videret

In tanto supplicio?

\section{IV.}

Jesus nachkiskawäu okiän koskänechtamenik.

Kit anamihätom...... As kes......

$\mathrm{Ne}$ nanakatawänechton omos, kaiesis kotakänechtach Jesus, as kes nachkiskawazin okiän iachpez tapanazin. Kätin kes käz wesakänechtam enechpe Jesus as näwazin okiän as kes monik. Ächpez tapanazin, enech ächpez wesakänechtach otähe. 


\section{3}

Jesus käshiateseon, ki käz tapanawak pämatesitoa achkihe; ene kaies io kotakechtawon omos achkihe, ächpez tapasea. Ene äsis natawänechtamon tä iochpe, Jesus, asau meseach waieskewach tapanitwon, opäsik asau tapanenon, mawau miniu nez pämatesitoa wäwänin asau tapanakoa, kenach io. Amen.

Nochnenau ... . Kit anomächkon...... Käz achpechtänetakosit.

Sawänemeach...... Hawätok kitämakänemeach......
Quis non posset contristari,
Christi Matrem contemplari
Dolentem cum Filio?

$\mathrm{V}$.

Simon Sirene wächpiat tachkonäu anamihäwachtikon.

Kit anamihätom...... As kes......

Ne nanakatawänechton omos, kaies esekit Jesus as kes iachpez anawechtok as pemenekonazin ot anamihäwachtikomon; ene tä Sutau inäniwak kaies io anonatoa Simohon Sirene kaies joch pianit, nitchia- 
54

kach asau inim tachkonazin Jesus ot ana* mihäwachtikon.

Jesus Täjänemeon, enoch inäniu kon o kes mininichtonon asau láchkonazin nemewach kit anamihäwachtikomon. Enech neu miniu nenach nap esekeon wawepoz; kon ne mänitähäon kon net otachpenarion sänakach kaies meseon. Jochpe tä wäwänin mawau nenau otachpenam. Au enänemeon ene kakik au esekeon. Amen.

Nochnenau ..... Kit anomächkon..... Käz achpechtänetakosit......

Sawänemeach..... Hawätok.....
Pro peccatis suae gentis
Vidit Jesum in tormentis,
Et flagellis subditum.

\section{VI.}

Käz Veronika kesechkonäu Jesuson.

Kit anamihätom...... As kes......

$\mathrm{Ne}$ nanakatawänechton omos, kaiesis mächno totawazin Jesuson käz Veronika. As mäk tä as käz kishisit Jesus as kes pemenekonazin ot anamiläwachtikomon enoch metämoch kes kisehämawäu, wapis- 
kikon kes au; ene tä enis wapiskikano Jesus oskesik kes masenechnin.

Jesus nähe sawänechzekäon, ne käz mininechton kaies pis isis sawänemech eno käz metämoch. Nenach nap ki katäu mächno echpänonin; ki kes etan; "Enekoch au echpänanekoa kez pämatesewak, nenach neu au echpänaseak." Ene wä mächno echpänanekoa achpäniu kez pämatesewak. Amen.

Nochnenau..... Kit anomächkon..... KKäz achpechtänetakosit......

Sawänemeach...... Hawätok......

$$
\begin{aligned}
& \text { Vidit suum dulcem Natum. } \\
& \text { Moriendo desolatum, } \\
& \text { Dum emisit spiritum. }
\end{aligned}
$$

\section{VII.}

Jesus nishino papechzin anamihäwachtik mihikone.

Kit anamihätoin...... As kes......

$\mathrm{Ne}$ nanakatawänechton omos, kaiesis käz anemesit Jesus, misik kes papechzin achkibe; ene nishino kes papechzin; kon 


\section{6}

tä pachpis semakanechson o kes sawänemekonon; kes papakomik, kes tachkiskak miniu, asau nipitat.

Jesus Täpänemeon, ki mamachkatänemin kaies tapachnänichsion, kaiesis anemeseon miniu, nenach io. Osam ne mamenatesin, osam miniu achpäniu nasop ne papechzenäm päzetone, ene iachpez kaies io kotakechtawon, Jesus käshiateseon! Suwänemena, sochkitähäskawena, waieskewach mihikon achpäniu asau pemochneon. Amen.

Nochnenau..... Kit anomächknn...... Käz achpechtänetakosit......

Sawänemeach...... Hawätok......

Eja Mater, fons amoris,

Me sentire vim doloris

Fuc, ut tecum lugeam.

\section{VIII.}

Sherusalem tase metämochsok nawemäwak Jesuson.

Kit anamihätom...... As kes....

$\mathrm{Ne}$ nanakatawänechton omos, kaiesis sawänemazin Jesuson auech metämoch- 
sok. Kes nämuk mihikone mäse mächki, kaies ochzekawik Jesus as kes pemochnet; ene tä as kes sawänematoa kes nawemäwak.

Jesus nähe sawänechzekäon, kaies käz kotakechtawon omos achkihe as kes pis kitämakänimatoa pämatesitoa, kätin ne koskänechton mächkawänechtamone kaiesis käz kotakechtawon. Kikes sawänemenau, Jesus ki mawemim nitähe. Ne käz sechkaton päzeton. Ene kä ne päzetonon kaies io kotakechtawon omos achkihe. Amen.

Nochnenau..... Kit anomächkon......

Käz achpechtänetakosit......

Sawänemeach...... Hawätok......

Fac, nt ardeat cor meum

In amando Christum Deum,

Ut sibi complaceam.

\section{IX.}

Jesus nänino papechzin anamihäwachtik mitikone.

Kit anamihätom...... As kes......

$\mathrm{Ne}$ nanakatawänechton omos, kaiesis käz anemesit Jesus, misik kes papechzin 


\section{8}

as kes pemenekonazin ot anamihäwachtikomon; ene nänino kes papechzin. Kes nächkosewak tä semakanechsok kaies isianatoa Jesuson, kes papakamawak tä asau nipitanit. Näskäniu tä kes wichkichtau as nipitat. -

Jesus Täpänemeon, kätin netäkisim naiakatawänechtamonin kaiesis kotakechtawon, nenach io. Achpäniu päzetone ne papechzenäm, ene kä iachpez kaies io kotakechtawon, ene kä achpäniu kaies io papechzenon as kes pemenekonot kit anamihäwachtikom. Sochkitähäskawena Jesus, iochpe kon asau päzetaın. Amen.

Nochnenau..... Kit anomächkon.....

Käz achpechtänetakosit.....

Sawänemeach..... Hawätok.....

Sancta Mater, istud agas,

Crucifixi fige plagas

Cordi meo: valide.

$\mathrm{X}$.

Jesus machkamau o posächkanon.

Kit anamihätom...... As kes......

Ne nanakatawänechton omos, kaiesis maze echpänone Jesus as kes pia1ät wach- 
kitachkiach as kes tase sachsakachkoaho anamihäwachtiko. Semakanechsok tä mawau kes machkamäwak o posächkanon; ene tä echpe mawau weo kes mächkowit Jesus. Somenapo miniu kes menahäwak, wesop kes takonikatäk; kon tä Jesus o kes menänon.

Jesus keshiateseon, wawäremena, moska * wänichtamihina, kinä nesik kätau iachpez tapanenon, asau papamänechtamon omos achkihe ächtakik, asau mininechtamon kitämakeseu. Kon kenach käkoch omos achkihe tepenau ki kes tananon; pochz ki posächkanon ki kes machkamekim. Sawänemena, Jesus, kakik asau kächkenawapomenon. Amen.

Nochnenau..... Kit anomächkon..... Käz achpechtänetakosit......

Sawänemeach..... Hawätok.....

Tui Nati vulnerati,

Tam dignati pro me pati,

Pocnas mecum divide.

XI.

Jrsus sachsakachkohau anamihäwachtiko. Kit anamibätom..... As kes..... 
$\mathrm{Ne}$ nanakatawänechton omos, karesis käz kotakehe Jesus kaies ani machkame o posächkanon. Kes achpakenau wachkitachkiach, anamihäwachtiko tä kes sachsakachkohäwak, mämachkikim sakachikonon kes awak.

Jesus iachpez nawaneu esekeon, kon pochz nekotono ki kes kikitinon, as kes käz kotakehekäon, kon pochz ki kes keketotawanowanon maze ächpinonächkoa. Ne sechkaton ne weiatesin; ki pakichsenemin tä, Jesus Nochne, asau meseon nawaneu esekin, asau nawaneu esekoon kespin maze totakäon. Amen.

Nochnenau..... Kit anomächkon...... Käz achpechtänetakosit......

Sawänemeach...... Hawätok......

Fac me tecum pie flere,

Crucifixo condolere,

Donec ego vixero.

XII.

Jesus akozin anamihäwachtiko nipoa miniu. Kit anamihätom...... As kes......

$\mathrm{Ne}$ nanakitawänechton omos, kaiesis käz. wesakäncchtach Jesus, kinis kes ako- 
zin anamibäwachtiko. Kätin ne käz wesakänechtam enechpe; kon weiak pämatosit achkihe pos o wichkichtonon os pos kächkenach kaiesis kotakechtok Jesus anamihäwachtiko. Iskoaz tä kes nekizkichtau, ene tä as nepäk.

Jesus iachpez käshiateseon, kätin ki käz tapanin; ki pämatesin ki kes paketänechton, asau pemazeheon, kakik pämatesin kesekoch asau meseon. Änekochkitähäon ki mamiawamin Jesus Nochne. Wetochkawena wäwänin asau pämateseon, ene tä asau mächno nepäion. Amen.

Nochnenau..... Kit anomächkon......

Käz achpechtänetakosit......

Sawänemeaach.... Hawätok.....

Juxta Crucem tecum stare,

Et me tibi sociare

In planctu desidero.

\section{XIII.}

Jesus achtowau käz Marion onächkine.

Kit anamihätom...... As kes......

$\mathrm{Ne}$ nanakatawänechton omos, kaiesis menazehatoa Jesuson anech kaies mächno 
isikitoa inäniwak. Kaies nepäk Jesıs anamibäwachtiko kes otachpenamuk wean, käz Marion onächkine kes achtowäwak; ene tä wenach enechpe kes käz koskänechton.

Jesus Täpänemeon, mawau keau kes mächkoweu as kes paketenekäon kikia onächke. Ki pakichsenemin, Jesus, asau ponikitätaweon mawau ne maze esetonon; as kes sekinamon mawau ki mächkom, nenach io, ene wä pakichsenemenon, asau kisehamaweon mawau maze esotonon. Amen.

Nochnenau..... Kit anomächkon...... Käz achpechtänetakosit......

Sawänemeach...... Hawätok......

Virgo virginum praeclara,

Mihi jam non sis amara,

Fac me tecum plangere.

\section{XIV.}

Jesus wäwänin paketänemau.

Kit anamihätom...... As kes.....

$\mathrm{Ne}$ nanakatawänechton omos, kaies mächno echpänonatoa Jesuson kaies iach- 
pez mächno isikitoa, as kes pakitänimatoa wäwänin. Kätin Jesus kes nipoa anamihäwachtiko, kakik pämatesin asau menenach kesekoch. Misik tä kes iach pemateseu as inim näno konakach, ene tä iochpe kesekoch as pematesit kakik.

$\rightarrow$ Jesus Nochne, ächpez tapaseon ki kes paketänechton ki pämatesin, as katäu pemazeheon. $\mathrm{Ki}$ pakichsenemin, Jesus pinichtowena netäh, kakik tä netähe pämatesenon. Kanawänemena asau mächno pämateseon, kon asau wanechnetawon kakik pämatesin kaiesis wichkichtoweon as kes pis kotakechtawon. Amen.

Nochnenau ..... Kit anomächkon..... Käz achpechtänetakosit.....

Swänemeach..... Hawätok......

Fac ut portem Christi mortem,

Passionis tac consortem,

Et plagas recolere.

\section{Anamihan.}

Jesus, kaies iachpez sawänematoa pämatesitoa, kätin änekochkitähäon ki mamiawamin eneko kaiesis sawänemeon nenach 


\section{4}

nap. Kit anemesin ki käz kotakechton miniu, ene naszeau kätau kisehamakoseon, ne päz esekinon; ki näpin tä, ki iach pematesim miniu, ene naszeau kat io wichkichtoeon, kakik pämatesin kesekoch asau pichtikäon, kakik enis asau nianon. Amen.

Naszeau Hawätok sawänemäu kisichnasoane äpenit, wepoz asau otachpenazin kesekoch. Amen.

Jesus Täpänemeon, sawäneme mawau kisichnasoane äpitoa; akoachsemin äs kotakänichtakoa; kakik wanachkione kesekoch asit. Amen.

Nekotoasita "Nochnenau"..... "Kit anomächkon"..... "Käz achpechtänetakosit".

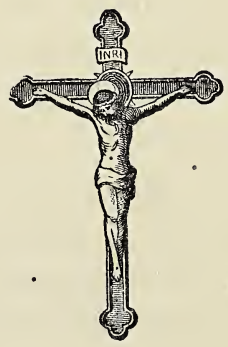


65

\section{II.}

\section{SIKAHAPUANITWON. \\ Kakichkotakan.}

Sikahapuanitwon opäsik kätau käz enapazechtok. Kon saikahapuasit kon pos o wichkichtonon kesekoch asau isiat kes anich pematesit achkihe. Mawau päzetonon kisehekatäwon saikahapuanekäiachkin, päzeton pis kikeiochtateseach, mawau. miniu eneko kaies pis päzetaiach konomächtchiu as sikahapuanekäiach. Echpe änamihaiach kaies sikahapuanekäiach, Hawätok miniu wänitchionächsemeach; Waieskesit Hawätok miniu ki pichtikakonau enechpe. Täpänechzekät enänemik Mäıhkotächkoniäu asau sikahapuanekot. Weiak tä kon saikahapuasit konomächtchiu, kaiäz wesakesit tä, kächze kon asit Mächkotächkoniäu, mawau pämatesitoa pos wichkichtawak asau sikahapuanatoa. Nekotono nesik weiak wäwänin au sikabapuanau. Ene tä au sikahapuanekot kon Mächkotächkoniäwon aiawenit, ene näwazin Mächkotächkoniäwon, ene asau 


\section{6}

natotamowazin, wäwänin asau sikahapuanekot. ' Ene kä nekotono nesik wä wichkichtawach, asau sikahapuanekäiach. Kitätchiakonawak kächkewächzekasowak echpe saikahapuanekäiach, kon tä nekotoch kisechkawon ene äsis kächkewächzekasitoa.

Mächkotächkoniäu ene wä esetot. Konomächtchiu saikahapuasit weiak, kon kätin ot anamihanon, kon Hawätokon ot änänemekonon wänitchionächse. Ene tä wä esetot asau mächkawänechtomach. Kitätchiakonawak asau io inänitoa onamichkon $V$ tamoan, sikahapuanitwon miniu, $V$ nekotoch asau panatesit, kakik pematesin asau mene, kespin mächno kanawichtowazin Hawätokon o sawänechzekon, kaiesis menekot echpe sikahapuane. Misik tä Mächkotächkoniäu wä anamihäwachtikonomowazin, asau mächkawänemazin Jesuson, anamihäwachtiko kaies tachpänänit; asau kon nekotoch tikisetach minịu Jesusct Anamihan. Misik tä Mächkotächkoniäu wä pichtikawazin sikahapuanazin anamihäwigamiko asau kächkenomä: mawau ha 
saikahapuasitoa kesekoch asau pichtiketoa kes anich pematesitoa omos achkihe, äsis pichtiketoa iochpe anamihäwigamiko, kespin mächno kanawechtokoa Hawätok o sawänechzekon asau mächno äsikitoa. Misik tä Mächkotächkoniäu wä anamihäwachtikonumowazin ochtawakone otone miniu sikahapuanazin asau mächkawänemazin: saikahapuasit käz aiakoamemau kon asau pächta maze kikitwon kon asau maze kikitit miniu wenach.

Misik tä Mächkotächkoniäu wä anamihäwachtikonomowazin ochpaniach, tepach otähe, änekoch lukitähät asau tapanat ot Anamihan; misik nanakiten tä, sinawaneu asau kotakänechtach, käkoch äsikitehin, kaiesis kotakänechtach miniu wenach Jesus as kes pemenekonazin ot anamihäwachtikomon. Misik tä Mächkotächkoniäu wä hatok wapiskikon wene kaies sikahapuanazin, asau isis kächkenamach : iochpe kaies sikahapuasit otätchiakon penesewon, wapiskikon äsis penach; kon käkoch päzeton iochpe o käkiskanon mawau päzetonon kisehekatäu. Iskoaz 
68

tä Mächkotächkoniäu wä menazin wasachkonenakon, asau isis kächkenamach: mawau kon saikahapuasitoa kiskanitipächkatoa tase pematesewak; akiko tä saikahapuasitoa, wäwänin miniu kächkinachkoa Anamihan, Jesus ot Anamihan, wachsinakwatoa tase pematesewak. Ene tepa wasachkonenekon kotänos säkächnasamächkin, mawau wasachkoneu.

Tächkonatoa, wänitchionächsirnatoa, äs wechtek, kätäu sikahapuaneme, asau kakichkimatoa o nitchionächsowawon asau nipitachnatoa asau kächkenamänik Katolik Anamihan.

Echpe Mächkotächkoniäu piat asau sikahapuanazin nitchion wepoz'kozemonäu :

Mächkotächkoniäu: "Wäki ki natawänechtamon Hawätok ot anamihäwigamik?"

Wänitchionächsematoa: "Papamächtamon."

Mächk.: "Wäki maienekäion papamächtamon?"

Wänitchi: "Kakik pematesin."

Nawenau tä tächkonatoa pos wechta- 
mowäwak "Nepapamächtawau Hawätok Wäwochneme," "Nochnenau kesekoch äpeon." Ene Mächk.: "Ki pakitänämit maze Hawätok?"

Wänitch.: "Ne pakitänau."

Mächk.: "Ki pakitänämit mawau ot anochkiwinon?"

Wänitch.: "Ne pakitänan."

Mächk.: "Ki pakitänämit mawau ot onanekosinon?"

Wänitch.: Ne pakitänan."

Mächk.: "Ki papamächtawau Hawätok tatachkesit Wäwochneme kaies kesechtok kesik achkäu miniu?"

Wänitch.: "Ne papamächton."

Mächk.: "Ki papamächtawau Jesus Hawätok Wäkichseme nekoneneu, Täpänemenach, kaies pis ochtatesit, kaies kotakechtok kinach io?"

Wänitch.: "Ne papamächton."

Mächk.: "Ki papamächtawau Waieskesit Hawätok, käz Katholik Anamihäwigamik, käz achpichtisitoa o wetochkatoanowau, päzetonon kisehekatäwon, iach apiskilk weau, kakik pematesin?" 
Wänitch.: "Ne papamächton."

Mächk.: "Ki natawänechtämit asau sikahapuanekäon?"

Wänitch.: "Ne natawänechton." Anamihanon

Hawätok mesas käkoch nähechtawon, ki mamiawamekon as kes kanaweheach as kes änamihaiach. Kakik sawänemeach, kakik kinach papamächtakäm au äz pemateseach. Amen.

Kinach waiez owichswonemenach, keketotamoweach, kaiesis anamihaion achkihe, nenach nap tä asau isis anamihaion kakik tä mmiu kesekoch asau wekemenach kes nepäion achkihe.: Amen.

Käz Mishael, kinach Mäz Okemau mawau așäniwon, kinach kaiäz asäniweon, kinach kaies nochkehat maze hawätok, wetochkaweach, nenach nap asau nochkehake saiekaseamä. Amen.

KAKICHKOTAKAN OS POS NENOCH. TAMÄ WÄW ÄNIN OS POS SIKAIIAPUANE.

Täpänechzekänit enänimik MärhknŁächkoniäu asau sikahapuanckot. Iïäz 


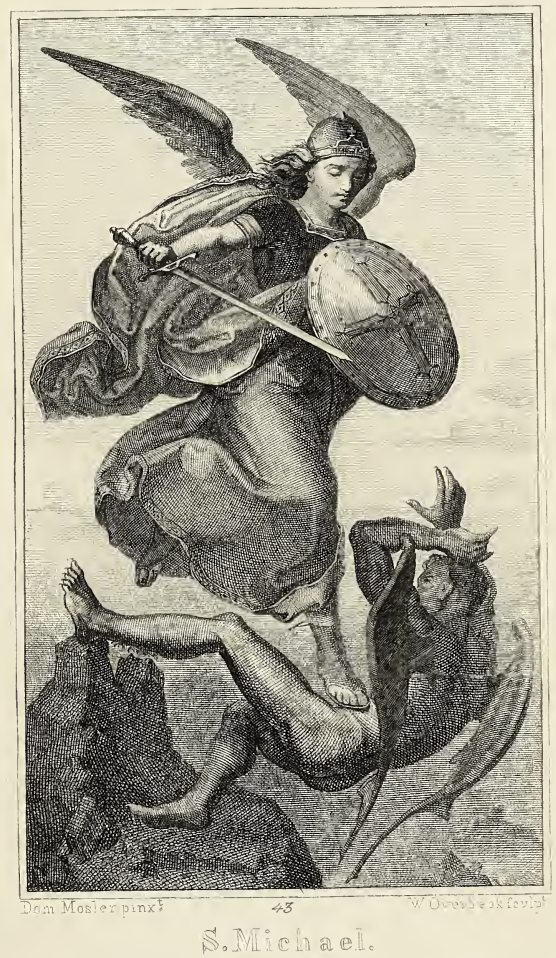

Eigenthum des Vereins zur Verbreitung relig;iilderinDüfed os:f

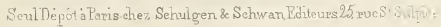



achpechtänechtakwot Sikahapuanitwon. Kon weiak kesekoch pos ot isianon kespin kat sikahapuasit.

Ene pos.wä sikahapuasit oske nitchion wepoz os pos nepäk konomächtchiu asau sikahapuasit. Kaiäz wesakesizin tä oske nitchion konomächtchiu sikahapuazikasit, kespin as katäu ani pematesit, papik pos kächkewäiapawanau. Mawau neu wichkichtawak pämatesitoa os pos kächkewäiapawanatoa oske nitchionon kaiäz wesakesinizin. Kespin tä miniu näwazin kat änamihat onitchionechsowawon katäu näpenik, pos kächkewäiapawanau wepoz äs wichkichtawon. Jachpez tä käz achpichtänichtakwot kächkewäiapawazekon. Kes nepäk tä oske nitchion kaies kächkewäiapawone, kesekoch au isiu, ene tä kakik as pos sawänechtakosit. Kes nepäk tä kat kaies mächno kächkewäiapawazikasit kon rekotoch pos o pichtikenon Hawätok ot okemawine kesekoch. Ene tä au esechzekäion, asau mächno kächkewäiapawanot oske nitchion.' Otachpenach acpeu sekenaumowin tä nitchion ochkäch- 
ne, konopoz miniu wene, as mäk sikahapuane konau eta: "Ki sikahapuanin o wichswone Wäwochneme misik Wäkiseme misik Waieskesit Hawätok.66 .

Wäwänin sekenach nepeu asau wakichsenit onoke.66

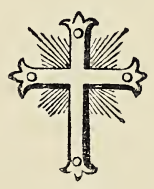


III.

\section{SOCHKITÄHÄSKATWON.}

\section{Kakichkotakan.}

Misik käz Sawänechtakosin ene Sochkitähäskatwon. Käz ächpechtänechtakwot Sochkitähäskatwon. Aiom käz Sawänechtakosin kä wätachpenachkin, ki pichtikakonau Waieskesit Hawätok, ki menekonau o sawänechzekon; ki moskawänechtamihikonau miniu, wäwänin asau onamichtamach Jesus o kakichkotakan, wäwänin miniu asau esetaiach eneko Jesus kaiesis aiakoamechnesit.

Ki pichtikakonau Waieskesit Hawätok echpe saikahapuanikäiach; Sochkitähäskatwon tä maienikäiach, opäsik ki moskinanikonau Waieskesit Hawätok o sawänechzekon. Nohekon tachnäno kit äsis sawänemekonau:

1. Waieskesit Hawätok ki kakitawehekonau, ki kächkenohamakonau ächpez Waieskesit Hawätok Näniu Nekot aweu, ächpez miniu onanekoch kesekoch. 
2. Ki menekonaû nipoachkon, wäwänin asau nenochtamach Jesus o kakichkotakan.

3. Ki kächkenohamakonau au esetaiach käkoch sänakach äsikeachkin.

4. Ki sochkitähäskawenemekonau, asau wichkichtawach wäwänin asau esetaiach änenach Hawätok.

5. Ki moskawänichtamihikonau, kon asau tikisetamach kit anımihamenau, wäwänin miniu asau kächkenamach au esetaiach, kwaiachk asau pemateseach.

6. Ki pechnahamakonau kit änänechtamowenach, asau tapanake Hawätok, wäwänin miniu asau anamihaiach.

7. Ki moskawänichtamihikonau, kon asau kotamach nepä miniu asau kotakehekäiach, naskat miniu asau nächnekäiach, Jesus ot Anamihan asalu ochzenanikäiach, asau kotamach tä päzeton.

Sochki ähäskatwon miniu ki totakoian asıu iachpez sochkänechtamach äsis anochkitawakeHawätok, as menechsenowatese miniu asau mekanakemaze hawä- 
tok, kon asau sakozehe nanas asau nepäion.

Misik tä Mäz Mächkotächkoniäu nesik wichkichtau as mikit Sochkitähäskatwon.

Weiak tä nekotono nesik wichkichtau pos otachpenach iom käz Sawänechtakosin. Ene kä nekotono nesik wä wichkichtawach asau menekäiach Sochkitähäskatwon : asau kitätchiakonawak äsis kächkiwächzikasitoa, echpe maienekäiach Sochkitähäskatwon, kon tä nekotoch pos kisechkawon ene äsis kächkiwächzekaseach. Ene tä kon sochkanamihatoa, kon o papamächtawanowawon Waieskesit Hawätokon, otähowa aiano keketotakotoa.

Erie kä au esetot weiak echpech otachpenach iom käz Sawäncchtakosin. Wäwänin encchpe au aninechso, au paketchekäu; au pininakwot äkit; kon miniu anamihäwigamiko mesewäu onau anıpenon; wäwänin au anamihau, au hewa miniu anamihan ätamä, kowomächtchiu mene Sochkitähäskatwon. Ene kä ätamä :

Hawätok Nochne, ki papamächton. Net onamichton minin mawau kaiesis kächkenohamakäion äsis anamihaion. 
Kit anomächkon Waieskeseon Hawätok! Ki pihin asan pichtikaweon nitähe, asau moskinäskakoian sawänechzekon. $\mathrm{Ki}$ mamachkatänemin ächpez sawänemeon, ano ki kes nächkehe. Wächzetau kätin net aninichsim eneko kaiesis päzetaion; wächzetau kätin ki tapanin änekochkitähäion, naszeau kakik asau apetaweon netähe; naszeau kat ki sawänemim kaiesis osetachseon Jesus. Amen.

Tächninächtchitawäu wene maienazin Sochkitähäskatwon, Mäz Mächkotächkoniäu keketotamowäu, änamihätawäu, anamihäwachtikonamowäu ochkächne, mänawaz tä miniu pakamowä)äu.

Mäz Mächkotächkoniäu mamiachtomäu enechpe Waieskesinit Hawätokon, asau pichtikakonit anino maienazin Sochkitähäskatwon.

Echpe Mäz Mächkotächkoniäu taiächninachtchitoch, keketotamowazin miniuenechpe paichtikakonit Waieskesinit Hawätok.

Mäz Mächkotächkoniäu tä wä anamihäwachtikonamowazin ochkächne, kon 
nekotoch asau tikisetach Jesus ot Anamihan.

Mäz Mächkotächkoniäu tä wä pakamowähazin mänawaz, asau kächkenohamonach mamawau, kon asau nächkänimakichtoa nächkäniminachkoa,sinawaneu miniu achpäniu asau kotakänechtamach käkoch äsikeachkin, naskat miniu asau nächnekäiach, Hawätok miniu o kikitwon asau ochzenänekäiach.

Pemäzenakach tä ene au otchichtchikoanekapawen, asau mamiawamat Hawätokon, au inänechtom tä: "Kätin ne sawänechtakosim as kes menekäion Sochkitähäskatwon; kon nekotoch nenau wanä- $n$ nechtanon net Anamihan." Ene tä iskoaz asau eta ene anamihan ätamä kaies ani miniti Sochkitähäskatwon :

Kit anomächkon, Waieskeseon Hawätok! Ki mamiawamin kaiesis sawänemeon iochpe, Sochkitähäskatwon as kes menekäion. Ki mänazehin änekochkitähäon. Ki mamiachtomin asau sawänemeon, kakik kesekoch asau onanekoseon. Kanawehenach asau kon käkoch nochkehe- 
78

koian net änänechtamoane mäte: sawänemena asau mächno pemateseon, kes nepäion tä, kesekoch kakik asau wez tanachkiamenon. Amen.

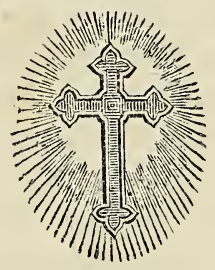




\section{IV.}

\section{PAKETCHEKON.}

Kakichkotakan asau nenochtamü ax esetach zе wäwänin asau paketchelä.

Paketchekäiachkin ene as kisehamachkaiach ki päzetoninawon, eneko kaiesis maze esetaiach kaies äz sikahapuanekäiach. Kon tachnäno weiak päketchekäzin kisehekatäwon o päzetonon; eno nesik änekochkitähät aianinichsit, wäwänin tä miniu paketcheket, enoch au kisehekatäkin o päzetonon.

Jesus kes osechtok en Paketchekon, $\varepsilon$ io kaies enazin o Mächkotächkoniäwon: "Hawänetowak au kisehamowäk o päzetonon, eno au kisehekatäkin; hawänetowak kat au kisehamowäk o päzetonon, kon au kisehekatäwon." Misik tä Jesus kes enäu o Mächkotächkoniäwon: "Enekoch au kächpetawàk ios achkihe, au kächpezckatäu kesekoch; enekoch tä au wapenamäk ios achkihe au wapinikatäu tä miniu kesekoch. Jesus tä kes menäu mawau Mächkotächkoniäwon, enee as kes eta, kiseheka- 
täwon, asau wichkichtokoa asau kisehamawatoa wäwänin paketchekänit o päzetonon; Jesus o wichswone asau io kisehamawatoa. Nianon ene pos äsetot weiak wä wänin asau paketcheket asau kisehekatäkin o päzetonon: 1. Pos nanakatawänechso ; 2. Pos aninichso ; 3. Pos enänechtam: kon misik nenach nekotoch asau päzetaion; 4. Mawau neu eneko kaies maze esetot au wechtamowäu Mächkotächkoniäwon; 5. Wäwänin miniu au kotakihiso as kes päzetot.

Pon eta: Nawenau nenau aninichsim. Iochpe wepoz aninichsinon, wawepoz miniu aninichsinon, wawepoz miniu paketchekenon, opäsik tä kinau wichkichton asau mächno esekeon, kinau sawänemik Hawätok. Kon॰mächtchiu nasächkawat Mächkotächkoniäu, nanakatawänichsinon iachpez wäwänin asau kächkenamon mawau ki päzetonon tachnäno miniu kaiesis päzetaion kaies äz paketchekäon aiatchkwat. Ene au io pakichsenemot Hawätok, asau sawäneme, asau wachsinakwot asau mene miniu wäwänin asau mächkawänechtamon mawau kaiesis päzetaion. 


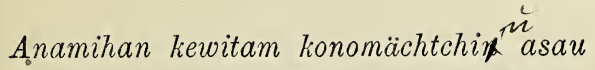
nanakatawänichsi asau paketchekä.

Hawätok Nochne, papik neu kit äsis mininechton asau mikion ki sawänechzekon, asau ponikitätawatoa tä miniu päzetatoa, kespin wäwänin aianinichsitoa. Sawänemena zenopomina, ne käz maze esekesin, wawepoz ano tä änekochkitähäion net aninichsim, ene wä pakichsenemenon asau kisehamaweon mawau ne maze esekenon. Hawätok Nochne, sawänemena, wasinakichtamihina, wäwänin mawau neu asau mächkawänechtamon kaiesis päzetaion. Sochkänichtamihina asau sechkatamon mawau neu ne päzetonon, kon käkoch asau isis sechkatamon, äsis sechkatamon ne päzetonon. Sawänemena, wetochkawänemena, iachpez kwaiachk asau katäu kächkenamon mawau ne päzetonon, au isis kächkenamon mawau neu kes nepäion, echpech tä au tepachkonikäu kesekach. Mächkawänemena eneko kaies maze esétaion, enekoch tä miniu kaies ano mächkawänechtamon os pos kes mächno 


\section{2}

esetaion, kon tä as kes esetaion. Mächkawänechtamihina eneko zikes nächkehenon. Amen.

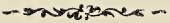

Ene iochpe mächkawänechtamon mawau neu ki päzetonon. Opäsik tä wäwänin mawau neu asau mächkawänechtamon kaiesis päzetaion kaies äz paketchekäion aiatchkwot, ene au akechtamon, konau tä nanakatawänichsinon wäwänin.

\section{NANAKATA WÄNICHSWON.}

Ta pe kaies paketchekäon aiatchkwot?

$\mathrm{Ki}$ kes wechtamowau tä Mächkotächkoniäu enechpe mawau neu ki päzetonon?

Wächzetau neu ki kes kiatoa Mächkotächkoniäu päzeton, as kes tikision as kes säkeseon?

Wäki kaies kiatawon?

Ta pe kaies äz kiatowat Mächkotächkoniäwon ene päzeton?

Ta tachnäno kaies paketchekäion kaies äz kiatawon ene päzeton? Ta tachnäno miniu kaies otachpenamon Jesus Weau? 


\section{3}

Ki kes wechtamowau tä Mächkotächkoniäu tachnäno kaies päzetaion?

$\mathrm{Ki}$ kes io koshkänechtamit tä ki päzetonon, iskoaz as kos päketchekäon?

Ki kes wichkoazichtomit tä wächzetau asau sechkatamon päzeton, nächkosin, kichkachtwon, maze kikitwon, kiwaskipiwin?

Mawau tä ki kes etämit anamihanon, Mächkotächkoninäu kaies mene?

\section{HAWÄTOK OT AIAKOAMECHNE- SINON. \\ I.}

- Nekoneneu Hawätok, mesas käkoch kaies kesechtok; nekoneneu kinau menazehau, änekochkitäkäon nekoneneu kinau tapanau."

Ki kes anamihamit tä wäwänin tachnäno mip nächkachkin tä miniu ?

Ki kes etämit tä: "kon nena Katolik net äsis anamihanon?" "kon ne pächtawanon Mächkotächkoniäu?" Ta tachnäno?

$\mathrm{Ki}$ kes tikisetämit tä Katolik Anamihan äsitoa kaiänit äsichzikätoa? Ta tachnäno? 
84

Ki kes isianit tä as tase kakichkotakät inäniu? Ta tachnäno?

Ki kes akechtämit tä kaiänit äsichzikämakach masenachigon? Ta tachnäno?

$\mathrm{Ki}$ kächkenämit tä pakuanau anamihanon ene kä: "Nochnenau kesekoch äpeon," "Kit anomächlion Maria," "Ne papamächtawau Hawätok Wäwochneme," Aninichswon miniu?

Nekotoasita ene pos kätau kächkenamä, pos kätau papamächtach miniu änamihat asau wichkichtok kesekoch os pos isiat; ki kächkenämit tä?

Ki kes kikäskämit tä maze maskichkiu tepa äsichzikätoa kat änamihatoa?

II.

"Kon nasop Hawätok kinau wawechnanon."

Ki kes maze kikitimit tä käkoch mochkoman kikitwon? (cursing, swearing) I'a tachnäno?

\section{III.}

"Anamihäkesegot kinau menazechton."

Kinis tä ki kes anochkimit as Anamihäkesekachkin, as Käz Kesekachkin miniu. Ta Tachnäno? 
Kisähächkoa ki kes pemochnemit Käz Kesekachkin? Ki kes pachpesemit? Kikes namäsächkämit? Ta tachnäno?

$\mathrm{Ki}$ kes ishiamit as tase menä iskotäu wapo, as tase atatich miniu? Ta tachnäno?

$\mathrm{Ki}$ kes ishiamit tä as tase anamiha tachnäno A namihäkesekachkin, tachnäno Käz Kesekachkin miniu?

Kat as wichkichtawon wä $\cdot$ hzetau neu konä kikes ishianon as tase anamiha Anamihäkesekachkin Käz Kesekachkin miniu?

Wäwänin ki kes anamihamit tä ächpez Mäz Anamihan?

Mesas kikes anapemit ana mihäwigamiko pichtik äpitoa? Kikes aiänemit tä? Ta tachnäno?

$\mathrm{Ki}$ kes ishiamit tä as tase anamiha nächkachkin (Vespers) Anamihäkesekachkin Käz Kesekachkin miniu?

Nochpeme waiekeianin, kespin miniu, kat asit Mächkotächkoniän Käz Kesekach ki kes anamihamit keke?

Ki kes akechtämit tä Mäz Anamihan?

$\mathrm{Ki}$ kes akemawakit tä anamihäwigamiko? 
86

\section{IV.}

"Kochne, kikia miniu kinau menazehawak, kinis asau pemateseon omos achkihe."

Ki kes papamächtawawakit tä kinekehekok?

Ki kes käz ki nochtamawakit tä? Ta tachnäno?

Ki kes mächno totawawakit tä achpäniu?

Ki kes kanawehawakit, kinekehekok?

Ki kes sawänemawakit tä miniu mämik echpe kätemakesitoawin, waiesakesitoawin käz kitoanin?

Ki kes maze nachkomawakit tä käkoch as inächkoa? Ta tachnäno?

Ki kes käz nächkimawakit tä kinekehekok? Ta tachnäno?

Ki kes äz nächkehawakit, käkoch as kes maze esetaion? Ta tachnäno?

Ki kes pachpinotawakit tä kinekehekok? Ta tachnäno?

Ki kes maze tasemawakit tä? Ta tachnäno?

Ki kes inänemawakit tä, nas tä kat anich pematesewak? Ta tachnäno? 
Waiesakesitowin kinekehekok, ki kes pis natoma tä Mächkotächkoniäu, maskichkiu inäniu miniu?

Kaies anich pematesitoa kinekehekok, ki kes keketotamawakit tä iachpez neu wäwänin, wawepoz miniu?

Ene io pos nanakatawänichsitoa wänitchionechsitoa.

Kaies ani ochtatesit nitchion, wepoz ki sikahapuana tä?

Ki pämihawakit tä wäwänin ki nitchionechsok?

Ki kes kächkenohamawakit tä ki nitchionechsok anamihanon?

Wäwänin anamihawakit tä ki nitchionechsok tachnäno mip nächkachkin miniu?

Mawau ki kes enäsehawakit ki nitchionechsok as tase anamiha tachnäno Anamihäkesekachkin?

Ki kes paketenawakit tä asau anamihanitoa ene tä asau io isiatoa anamihäwigamiko Anamihäkesekachkin Käz Kesekachkin miniu?

Ki kes kehomawakit tä asau paketcheketoa asau otachpenachkoa tä miniu J esus Weau? 
88

Ki kes aninimawakiı tä wäwänin, ki kes säsiwahawakit tä miniu käkoch as maze isichziketoa?

Ki kes kinuawawakit tä kat asau witchiwatoa maze nitchionon?

$\mathrm{Ki}$ kes paketenawakit tä naskat asau isiatoa as tase nimiheti, as tase menä tä miniu?

Ki kes paketenawakit tä kikisok, kitanok tä miniu asau witchiwatoa päsikoaz kikitinit, päsikoaz esetonit tä minin? Ta tachnäno?

$\mathrm{Ki}$ kes paketenawakit tä asau winochkawatoa kaiänit äsechzekänit kat änamihanit tä miniu?

Ki kes paketenawakit tä asau wekematoa kaïnit äsechzekänit kat änamihanit miniu?

$\mathrm{Ki}$ kes maze wapachtänawakit tä ki nitchinechsok as kes maze kikition, as kes maze esetaion miniu? Ta tachnäno?

$$
\mathrm{V} \text {. }
$$

"Pon weiak näsin."

$\mathrm{Ki}$ kes käz nächkosemit tä? Ta tachnäno? 
Ki kes käz nächkema tä kez pemateseu? Ta tachnäno?

Ki kes kechkama tä weiak? Ta tachnäno?

Ki kes nächkema tä kez pemateseu konopoz miniu waiekemat? Ta tachnäno?

Ki kes käz pakama tä weiak? Ta tachnäno?

Ki kes sechkana tä weiak? Kinis tä?

Ki kes maze enämena weiak, nas tä kat kitämakeseu? Ta tachnäno?

Wächzetau tä ki kes pakamawakit ki nitchionechsok, as kes nächkoseon? Ta tachnäno?

$$
\begin{gathered}
\text { VI. } \\
\text { "Pon päsikoatesenon." } \\
\text { IX. }
\end{gathered}
$$

"Pon mochnawenawin kez pemateseu waiekemazin."

Kespin kat kächkenau Mächkotächkoniäu wechtamawin kina aiaweon, - ne wekemewäm, - konopoz miniu, kon ne wekemewänon, - ene au enat.

Ki kes päsikoatänemit tä wächzetau? Ta tachnäno? 
90

Achpäniu ki kes wichkoazichtomit tä asau paketamon päsikoaz inänichtamowin.

Ki kes mochnawenämit tä asau esechzekäon käkoch päsikoatach? Ta tachnäno?

Ki kes maze kikitimit tä? Ta tachnäno?

Mäse tä ki kes nochtakokit pämatesitoa as kes maze kikition?

Ki kes mininichtämit tä asau pächtamon maze kikitwon? Ta tachnäno?

Ki kes zenapachtämit tä wächzetau neu käkoch päsikoatach? T'a tachnäno?

Ki kes maze totawa tä weiak? Ki kes maze totawa tä waiekemewät? Kat waiekemewät? Ta tachnäno?

Ki kes maze wenochkawa tä weiak? Ta tachnäno?

Ki kes päsikoatesemit tä ? Ta tachnäno?

Ki wetochkawa tä kaiänit äsechzekät, konopoz miniu kat änamihat?

Kit anamihäu - wekemewämit tä? VII.

"Pon kimotenon."

$\mathrm{X}$.

"Pon weiak mochnawenamowin käkoch ächtanach."

Ki kes kimotemit tä käko mäse ächpichtänichtakoa? Ta tachnäno? 


\section{1}

Ki kes iach mena tä eno kaies kimotemot?

Ki kes panazechtowa tä käkoch kez pemateseu otin? Ta tachnäno?

Ki kes tepahamawa tä wäwänin eno kaies panazichtowat otin?

Ki kes waiäsema tä weiak? Ta tachnäno?

Osam mäse ki kes enakichtämit tä as tepahakäon? Ta tachnäno?

Ki kes tepahämit tä käko konau tä as inänechtamon: nawenas iachpez kon nekotoch, - nawenas kon nekotoch nenau tepahanon? Ta tachnäno?

Wäwänin tä ki täpahämit eneko masenachekäion?

Ki kes wawichtamawikimit tä atawäwikamiko: wepoz nenau tepaham iom, kon tä ki kes tepahanon, kaiesis wawichtamawikion? Ta tachnäno?

Ki kes änänechtämit tä käkoch asau panazichtäwat kez pemateseu otin? Ta tachnäno kaiesis maze enänechtamot?

Kesṕin käko kes mächkamon, ki kes iach mea eno kaies wanechnetok?

Kwaiachk tä ki kes tepahamowa kaies anochkitok? 
92

\section{VIII.}

"Pon kinochkenon, asau pachtamot weiak."

Ki kes kinochkimit? Ta tachnäno?

Ki kes kinochkewamawakit tä kinekehekok?

Ki kes maze tasemau ı̈̈ kez pemateseu?

Wächzetau tä ki kes tepachkona kitähe kez pemateseu? Ta tachnäno?

Kespin kes käz maze tasemat kez pemateseu, ki kes papamazema tä: ne kes kinochkim, as kes maze enak enoch inäniu, eno metämoch; kon ene maze o kes esetonon, kaiesis patamak?

Käz Mäihkotächkoniäwak ot aiakormechnesenon; misik tä käz päzetonon naiakanänitakoachkin.

Ki kes metchemit metchemächsä wächzetau Anamihäwachtik kesekach? Ta tachnäno.?

$\mathrm{Ki}$ kes metchemit tä metchemächsä wächzetau asau pächtawechne ächpez käz mesachkatawä konomächtchiu as otächzechne Pachk? Ta tachnäno?

$\mathrm{Ki}$ kes metchemit tä metchemechsä 
wächzetau Mari kesekach ächpez käz mesachkatawä, echpe äkäiach kat asau metche? Ta tachnäno?

$\mathrm{Ki}$ kes mesachkatawämit tä niu enoch metatach tachnokon, konomächtchiu as otächzechne Pachk, tachnäno tä miniu äkäiach asau mesachkatawäiach? Ta tachnokon kaies mesachkatawäon?

$\mathrm{Ki}$ kes paketchekämit $\mathrm{n} \epsilon \mathrm{koz}$ nekotono tachnäno pepon?

$\mathrm{Ki}$ kes otachpenamit tä Jesus Weau echpe wätächzechne Pachk?

$\mathrm{Ki}$ kes iachpez mamenatesemit tä? Ta tachnäno?

Ki kes käz tapachnenema tä kez pemateseu? Ta tachnäno?

Ki kes sawänema tä kaiäz kitämakesit; waiesakesit weiak ki kes nasächkawa tä, ki kes sawänema tä miniu?

Wächzetau tä mäse ki kes mitchichsimit asau io wesakeseon? Ta tachnäno?

Ki kes kiwaskipimit tä? Ta tachnäno?

$\mathrm{Ki}$ kes menaha tä kez pemateseu? Ta tachnäno?

Ki kes käz kakawänema tä weiak, as 
94

kes mächnoit kespin miniu käz tanach käkoch?

$\mathrm{Ki}$ kes mininichtämit tä, as näwat as koskänechtach kez pemateseu, kespin käkoch mäte as otäsächkakot?

Kinäch anochkimit tä? Ki tatakazichkimit tä iachpez neu? Ta tachnäno?

Ki kes kitämahawakit tä waiekemat ki nitchionechsok miniu, kes kätemahawak?

\section{ANINICHSWON. \\ Kakichkotakan.}

Kaies ani nanakatawänechtamon mawau ki päzetonon, iachpez änekochkitähäon wäwänin aninichsinon as kes päzetaion. Kätin iachpez achpichtänichtakwot aninichswon. Ano käz weskiwat nanakatawänichswon, käz achpichtänichtakwot miniu, opäsik tä achpichtänichtakwot aninichswon. Kon nekotoch pos kisehamuanon päzetot kespin kon aninichsit, nasop pak tcheket, kon pos ponikitätawanon, asit tä achpäniu päzetone tase pemateseu, kes nepäk tä kakik iskotia au wenoso. 
Enoch tä kwaiachk aianinichsit, enoch kä kätau kisehamuch as kes paketcheket; kes nepäck tä, kesekoch au isiu, kakik tä au käz sawänechtakoseu. Ene aio wichkoazichtawon, asau wichkichtawon ene waieskewach aninichswon, Hawätok asau sawäneme, asau ponikitätok miniu. Kwaiachk kitähe asau aninichsion, oйe $n$. aio esetaion :

Iachpez neu änekochkitähäon anamihanon. Mamiachtomin Täpänechzekät asau wasinakoa, wäwänin asau nenochtamon äsis mäte päzeton.

Konau tä pakichsenemin asau iazechtok kitäh, asau käz sechkatamon päzeton mawau miniu päzetonon asau io koskänechtamon, konau tä asau moskawänechtamon: kon nekotoch ne katäu päzetonon, kon nekotoch ne katäu nächkehenon Täpänechzekät.

Nanakatawänechtach äsikimakach päzeton. Päzetot opäsik achpechtänechton mänawaz waienach onanekosin pächsikoatach, mänawaz miniu iskotäwapo, achpechtänemazin wenach tepenau Hawä- 
96

tokon. Kätin iachpez maze echpänonäu päzetot Täpänechzekänit Hawätokon!

Misik tä ene kätau io mächkawänechtamon: Iachpez kwaiachk tepachkonikäu Hawätok; kon opäsik o katäu isis kotakehanon mäze esetanit, ene tä kaiesis wichkoazichtoasit as kes maze echpänone. Kätin iachpez kwaiachk tepachkonikäu Hawätok; kakik iskotia tä achpakenäu päzetonit, tepa nekot nesik ait käz päzeton echpe näpenik. 'Kätin iachpez matät päzeton!

Misik tä mächkawänechtach kaiesis mächno echpänone Hawätok. Ki kes menik keau miniu ki tätchiakon, mawau miniu eneko mänäseon ki kes menik. Ki kanawehik achpäniu, ki tapanik, wänitchiänä kit ächpänonik. Ki sawänemik, wawepoz miniu ki kes kisehamak ki maze esekinon. Aiano kes wichkoazichtoaseon ochkano kakik iskotia asau wenosion, kon tä anamachkiach ki kes acbpakenekonon, kaies iachpez kitämakäneme. Kätin iachpez käsiateseu! 
Wenach tä enoch, kaies kinochtamazin kaies nächkehazin, kaies maze totawat! Nanakatawänechtach äsis wesakänichtachkoa kakik iskotia wainositoa. Kätin kotamikoat! Anamachkiach tanäseu keau kakik, kespin päsikoaz esekion. Kes ani tanächnachtik keau metatach nekotoachk tachnäno pepon, kon tä au ponetamakaton ki wesakänichtamoan; kakikächkamik au tanäseu keau maze iskotia, kakikächkamik kinau wesakänechton, kakikächkamik kinau mom, kakikächkamik kinau ohohim, kakikächkamik kinau kotakechtok maze hawätokok! Kätin nanisanit anamachkiach! Kinach tä, ki kes wichkoazichtoasim kakik iskntia asau achpakinäkäon tachnäno kaies käz maze esetaion.

Ninitchionä, Ninitchionä, nanakatawänechtach ene wäwänin, kochsin Hawätok, kochtach kakik iskotäu, aninichsinon tä iachpez änekochkitähäon, koskänechtach miniu ki maze esetaion.

Misik tä mächkawänechtach kaiesis tapane Jesus. Kinach io kes wechsakänechtau monachigone, kes kipotchiso 


\section{8}

mächki; kinach io kes pachkäskawau, kes tepachkonäwak miniu, kes pächkoakinäzitawäwak miniu ; kinach io kes sächsewahau, mawau weau kes mächkoweu; kinach io kes pasachkipenäu minichsakächsinon; kinach io kes pemenekonäu anamihäwachtiko; näniu tepachikon tä kes akozin anamihä wachtik, kes käz wechsakänechtam nanas as kes nepäk. Kaies iachpez tapane, ene kaies io kotakechtok mawau ene, kakik iskotia asau akuaséme asit tä asau mene kakik pematesin kesekoch. Kinach tä, kon ki kes tapananon enoch kaies käz tapane, ki kes nächkehau, ki kes maze echpänonau, opäsik ki kes tapatan päsikoatesin, iskotäwapo menäm, anoz miniu maze onanekosin, kaiesis tapanazin Täpänemeon ki Hawätokon! Ene kätau io aninichsion, ene kätau jo koskänechtamon.

\section{Aninichswon Anamihanon.}

Hawätok Nochne, wächzetau net aninichsim as kes nächkehenon, as kez päzetaion. Wächzetau ki käz achpechtäneta- 
kosim, ene wä sechkatamon mawau neu ne maze esekinon, kon käkoch net äsis sechkatanon, äsis sechliatamon ne maze esekin; kinach wä sechkatamon, ächpez waieskeseon. Sawänemena, kisehamowena, Jesus o wichswone io kisehamowena. Ni kes kesänechton kon misik asau päzetaion. Wetochkawena, sawänemena, kon misik nenau esekenon; au enänemeon ene kakik au esekeon. Amen.

Kätin net aninichsim, Jesus Nochne käsiateseon. Metchemänemena kit änänechtamowene; pon paketänemena; nochtawena, nochtawena, sawänemena as kes päzetaion. Ki tapanin tä miniu ki konin.

Käz Marie, Hawätok Wäkiachseon, achpäniu ki keketotamowak päzetatoa naiatenisemikoa; nenach nap tä kaies käz päzetaion, ki pis natenisemin as koskänechtamon as kes päzetaion; keketotamowena, wäwänin asau paketchekäon iochpe, asau ponikitätawit Hawätok mawau neu eneko kaiesis nächkehak.

Ne mächno asänim, kinach känaweheon, ki kes nämenon mawau ne maze 
100

esetanon; keketotamowena, mawau asau mächkawänechtamon ne päzetonon, mawau miniu kwaiack asau iochpe paketchekäion. Amen.

\section{$\nLeftarrow$ \\ MOSKA WÄNECHTAMON. \\ Kakichkotakan.}

Kwaiachk aninichsinon, enoch kä moskawänechtach asau iazechtok o maze pematesin, asau ponechtok miniu mawau maze esekin. Ene kätau io pakichsenemazin Täpänechzekät, asau moskawänechtamihe, kon nekotoch asau päzetaion. Wächzetau neu sochkänechtach, achpäniu wäwänin asau anamihaion mämik neu tachnäno watächnekoianin maze änänechtamoan. Naiämänin kätau nächkoseanin, nächkihächkoa miniu kinitchionechsok, kanawänichsinon wäwänin kon asau nächkihächkoa. Kespin wawepoz maze kikition, kesänechtach kon nekotoch asau nächkehemot kez pemateseu. Kespin 
ncanekotochkin kes nächkemot, kes kechkamot miniu waiekemat, sechkatach iochpe, kon misik asau nächkoseon, kakik tä asau mächno keketotawat.

Pon nekotoch winaziminon, iachpez mäte ene; pon miniu pächtawin mäze kikitit, mäze tasemazin miniu. Kespin kes menäion wawepoz maze nepeu, sechkatach iochpe iachpez wäwänin, asau kon nekotoch menäion, kon asau pichtikemakach sikinıkäwigamiko. Kespin kes maze wenochkawat weiak naskat moskawänechtach iochpe iachpez änekochkitähäion, kon nekotoch misik asau wenochkawat, kon nekotoch asau witchiwat, misik tä kon nekotoch asau keketotawat. Eno kätau maze wenochkawat weiak, kon kwaiachk ot aninichsifon, n nasop neu paketchekeu, kon kisehamuanon o päzetonon, kes nepäk tä kakik iskotia au wenoso.

\section{ANAMIHANON.}

Hawätok Nochne, sawänemena asau zenapameon, osam ne maze esekin, nanichkaz ki kes nächkehin, kon net 
achpechtänetakosenon asau zenapameon, sawänemena tä, Jesus o kotakechion ioch kisehamawena ne päzetonon.

Kätin ne käz koskänechton as kes nächkehenon, ne sechkaton tä mawau neu ne maze esekinon; kon nekotoch ki katäu nächkehenonon, zeau wepoz asau nepäion konomächtchiu misik asau nächkehenon.

Moskawänichtamihena, sochkänichtamihena, asau ponechtawon, asau sechkatamon mawau nächkosin, taniwächtoan, menän. Kon pachpis ne katäu maze onanekosinon.

Mesena ki sawänechzekon, opäsik asau aninichsion, opäsik tä miniu asau tapanenon, Jesus wänäseon Nochne! Nas tä kon nekotorh kat ne kes wanechneton ki sawänechzekon, as kes päzetaion! Sawänechtakoseu cno kon nekotoch wänechnetok! Sawänemena kakik ki sawänechzekon asau tase pemateseon. Amen.

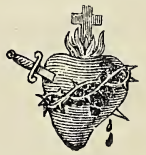




\section{PAKETCHEKON.}

Kakichkotakan.

Kaies iachpematesit Jesus, iom kes enäu o kächkenohamakanon, o mächkotächkoniäwon: "Hawänetok au kisehamowäk o päzetonon, eno kä au kisehekatäk. Hawänetok tä kon au kisehamowäk o päzetonon, kon au kisehekatäwon." (Joan.xx. 23.) Ene tä Mächkotächkoniäu ene wä wichkichtok asau kisehamawikit päzetonon, kespin päzetot kwaiachk aninichsit, konau tä nasächkawazin Mächkotächkoniäwon wechtamowazin mawau neu o päzetonon. Wäwänin asau wechtamowazin Mächkotächkoniäwon ki päzetonon, ene kä kätau esetaion:

Kaies ani nanakatawänechtamon ki päzetonon, kes ani aninichsion miniu as kes päzetaion, ene kanäu asau nasächkawat Mächkotächkoniäu. Piatäion tä as tase paketchek ä, of chichtchikoanetach iom tä esem Mächkotächkoniäu: "Keketotamowena, nochne, as kes päzetaion.” Anamihäwachtikonikenon, eta minıu ene pa- 


\section{4}

ketchekon anamihan: "Ne wechtamowau Hawätok mesas käkoch näkechtok." Nawenau tä wechtamowin Mächkotächkoniäu, echpe kaies paketchekäon iskoaz, konopoz nekot anamihäkesekach, konopoz nekot keso, konopoz nish keso, konopoz opäsik kinis.

Wäwänen ätotach ki päzetonon, tachnäno miniu kaies esetaion käkoch mäte wechtamowin Mächkotächkoniäu.

"Ni kes anamiham, eneko kaiesis mesit "Mächkotächkoniäu asau anamihaion.

"Kon ne katäu ision as tase anamiba "Anamihäkesekachkin nishino, wächze" tau net äsikin kon as kes.ision.

"Nänino ni kes käz kiwaskipim. Ni "kes käz maze tasemau nez pemateseu "metatach nenoch. Metchemechsä ni kes "metchim anamihäwachtik kesekach "nishino. Ni kes pächsikoatänechton "nianon neno."

Ene all isis paketchekäion. Achpäniu tä kinau atoton, tachnäno kaiesis päz änänechtamon, kaicsis päz kikition, kaiesis päz esetaion miniu, konopoz nekotono, 
konopoz nianon neno, konopoz nänino tachnäno kesekach.

Pon weiak wawechtach o wichswon. Kespin tä kozemone Mächkotächkoniäu, wäwänin nachkichtawim, onaminon, pon kinochkenon. Kon pematesit ki känochkewemanon, kiwanimeon Mächkotächlkoniäu, Hawätok ki känochkewemau. Pächtawin wäwänin Mächkotächkoniäu ene äne, kesänechtach miniu mawau asau katäu esetaion, enekoch äsis werhtamoch. Kes ani anamihätoch tä Mächkotächkoniäu, sakesinon as tase paketchekä, anamihäwigamiko tä anamihanon, mamiawamin enänemin Täpänechzekät, as kes kisehamuch ki päzetonon, misik tä anamihanon wäwänn, kaiesis wechtamuch Mächkotächkoniäu asau anamihaion.

\section{Mamiawamin Anamihanon.}

Jesus Nochne, ki kes enawak ki Mächkotächkuniäwak: "Hawänetok au kisehamawäk o päzetonon, ene kä au kisehekatäkin. Hawänetok tä kat au kisehamawäk o päzetonon, kon au kisehekatäwon." (Joal, xx. 23.) 
$100^{\circ}$

Ne iachpez achpänemim keau, iachpez ne pächton as kes kisehekaräkin ne päzetonon iochpe echpech anamihätawit Mächkotächkoniäu. Kaiesis kisehamawit Mächkotächkoniäu ne päzetonon ios achkihe, kit äsis pakichsenemin asau kisehamaweon kesekoch. Jesus Nochne, as kes inatoa ki Mächkotächkoniäwak, ene ächpänimion.

Änekochkitähäon ki mamiamawin, Jesus käshiateseon. As kes käz päzeraion no kes wichkoazichtoasimepach, anamachkiach kakik iskotia os pos kes tanächnasion; iochpe tä ki kes ponikitätawin ano as kes wichkichtoaseon kakik iskotia os pos kes tanächnaseon, kon tä ene ki katäu echpänasenon. Jesus Nochne, ki wichkoazchem kesekoch asau isiascon.

Ene tä änekochkitähäon wä aninichsion, as kächkenamon iochpe ki keshiatesewon. Wächzetau ne kes kesänechton, kon misik nasop enis ne katäu papechzenänon. Sawänemena tä miniu sochkänichtamihena. Amen.

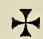




\section{7}

\section{MIAKONAMATWON SAWÄNECH- ZEKON. \\ Kakichkotakan.}

Nish käz Sawänechtakosinon kes menekonau Jesus, ene kä, Sikahapuanitwon misik tä Paketchekon, ene wä kisehamakiach ki päzetonenau.

Kespin käz maze esetot weiak, achpäniu kotachkechtowin anamachklach wichkoazichtoaso; kespın tä mänawaz nesik maze esetot, kon ächpäniu kotakechtowin wichkoazichtoas $\$$, ano 1 ä wichkoazichtoaso mi- inon niu wenach asau kotakechtok, nawenas ios achkiche, nawenas tä kisichnasoane. Weiak tä aianinichsit as paketcheket miniu, enechpechtä maiakonamo ene kakik kotakechtowin kaiesis wichkichtamasit, kes käz maze esetot. Ene nesik tä kakik kotakechtowin anamachkiach ächtakik as miakonamo; mitchineu tä änänechtakoseu asau kotakihisit ios achkihe; kespin tä ios achkihe wäwänin kon kotakihisit, au kotakechtau kisichnasoane. Au miakonama wau tä o kotakechtowin, kespin iachpez wäwänin kotakihisit omos achkihe, nawe- 
nas miniu wichkichtoasit, ene Miakonamatwon Sawänechzekon. Kespin weiak wichkichtoasit Miakonamatwon Sawänechzekon, miakonamawau kotakechtowin kaiesis wichkoazechtok ios achkihe, nawenas miniu kisichnasoane, as kes päzetot.

Kon tä päzetonon miakonamowanon, kotakechtowin nesik miakonamowau. Asau miakonamakäiach ki päzetonenau, Jesus kes menekonau Sikahapuanitwon, misik tä Paketchekon.

Käz Mäz Mächkotächkoniäu mamawau naikanisitoa menäu Kisehamatwon Sawänechzekon mawau änamihanit änekochkoa achkäu ainit.

Nish tä Kisehamatwon Sawänechzekon, ene tä: Käz Miakonamatwon Sawänechzekon, misik tä Miakonamatwon Sawanechzekonechson.

Kespin weiak wichkichtoasit Käz Kisehamatwon Sawänechzekon, mawau neu kotakechtowin miakonamowau, enekochkaiesis wichkoazichtoasit omos achkihe, misik tä kisichuasoane, as kes päzetot. Kespin weiak wichkichtoasit Kisehamat- 
won Sawänechzekonechson, anä tä kotakechtowin miakonamowau, ,nekoch kaiesis wichkoazichtoasit ios achkihe, misik tä kisichnasoane, as kes päzetot.

Näniu tä ene pos äsetot änamihat asau wichkichtoasit Kisehamatwon Sawänechzekon :

1. Wäwänin tä pos aninichso änekochkitähät tä, misik tä pos paketcheket.

2. Pos otachpenam miniu wäwänin käz Eucharistiwin.

3. Kwaiachk miniu pos esetau mawau neu kaies eta mamawau Käz Mäz Mächkotächkoniäu, echpe mesit Kisehamatwon Sawänechzekon.

Kon tä weiak nesik pematesit achkihe ot änapazechtonon Miakonamatwon Sawänechzekon, wichkichtau neanekotochkin änamihat asau menat ene sawänechzekon kisichnasoane ainit, akiko asau enapazechtokoa, wepoz tä kesekoch asau isiatoa. 


\section{V. \\ K ̈̈Z EUCHARISTIWIN, JESUS WEAU. \\ Kakichkotakan.}

Nanakatawänechtach iochpe, Ninitchionä, äsikimakach ene mamawaı käz Sawänechtakosin, ene Käz Eucharistiwin.

Jochpe kinau otachpenom ene mamawau käz Sawänechzekon. Jesus au pichtikäu kitähe; äsis Hawätokewit, äsis ma-. mazetawit miniu kinau isitäk, kitähe kätin au tanäseu. Kätin kinau käz Sawänechtakosin, wätachpenamonin Jesus Weau. Opäsik tä asau kächkenamon, äsis sawänechtako:it enoch wätachpenazin Jesuson Käz Eucharistiwine äpenit, kinau wechtamon kaies eta Täpänechzekät, as kes wawechtamaweko asau osechtok ene käz Sawänechtakosin. Pächta wäwänin metchemänechtach miniu: Iom tä kes enäu Jesua o kächkenohamakanon Sutau inäniwon miniu : 
"Kätin, kätin, kit äninämuau, enoch "wänamichtawit kanawechtau kakik pe"matesin. Nenach kä net awim enoch "pematesit pachkishigon. Kochnowawak " kes metchechsowak Manna enis pikoa" tachkamik; kes nepok tä. Eno kä pach" kishigon kesekoch wächpiat, ene tä kon " asau nepäk enoch au muazin. Nenach " aiaweion pematesit pachkishigon, kese"koch iochpion; kespin weiak muazin " aiom pachkishigon, au kakik pemateseu" ene tä enoch pachkishigon au mikion, "ene ne metchemechsim aio pematesitua "mamazetawak. Sutau inäniwak tä kes " käz keketotawak, iom kes hewak: 'Ane " aiom inäniu pos äsis wichkichtok o met" chemechsim os pos achsamenach?" Je"sus tä kes enäu: Kätin, kätin, kit äninä" muau; kespin kon mezäk pematesit Oki"son o metchemechsim, kon miniu me" näiäk o mächkom, kon pos kikäkiska " ninawon pematesin. Hawänetowak au " mezäk ene ne metchemechsim au menäk " miniu ene ne mächkom kanawechtau "kakik pematesin, nenach iach pemate 


\section{2}

"sehau miniu echpech iskoaz as kese"kach. Ne metchmechsim kätin mitchich"swon aweu, ne mächkom miniu kätin " menäu aweu.. Eno kä mitchichsit ne " metchemechsim, mänik miniu ne mäch" kom, käkiskak miniu wenach tä, ne kä"kiskau. Tepa kaies pis enäsehit Wá"wochneme pematesit, misik ne ioch "pematesim Wäwochneme; eno kä miniu "nenach maioat, nenach aio pematesit. "Eno kä pachkishigon kesekoch wäch"piat; au kikäskawäu wenach kaiesis " mitchikoa Manna kochnowawak, kes ne"pok tä; enoch kä au muazin aiom pach"kishigon, au kakik pemateseu." (Joan. vi, 47.-59.)

Kätin iachpez mininechtakwot Jesus o kikitwon. Enech neu miniu kenach kit äkoa Täpänechzekät :

"Nenach aiaweion enoch pematesit "pachkishigon... Kespin weiak muazin "anino pachkishigon, au kakik pemate"seu ... Hawänetok au mezäk ne metche" mechsim, au menäk miniu ne mächkom "kanawechtau kakik pematesim nenach 
"miniu iach pematesehau iskoaz as kese"kach."

Ene neu miniu ki natomik Täpänechzekät, asau muat eno pematesit pachkishigon kesekoch kaies iochpiat; kinach nap, kinau wechtamak Jesus asau mene kakik pematesin kesekoch, asau.iach pematesehe miniu iskoaz as kesekach, kespin wäwänin otachpenamonon Weau O Mächkom miniu Käz Tucharistiwine.

Jachpez tapane Jesus, ene kätau ioch pichtiket kitähe iochpe, ene kätau ioch achsame tepenau Weau. Kon weiak kit äsis tapanekonon, Jesus äsis tapane, misik tä kina kon weiak pos kit äsis tapananon, pos äsis tapanot Täpänechzekät. Wäwänin tä asau ołachpenamon ene mamawau kaiäz achpichtänichtakoa Sawänechtakosin, ene mächkawänechtamon au esetaion miniu.

Katäu otachpenamonin Käz Eucharistiwin, nanakatawänichsinon wäwänin, asau kächkenamon as pemateseon. Kespin tä kikäskamon mäz päzeton, aninichsinon paketchekonon miniu wäwänin, Hawätok 
asau kisehamoach mawau ki päzetonon. Ene wä asachzekatäk Hawätok o masenachigone: "Hawänetok au muazin anino "pachkishigon konemau miniu au menäk "enech ätik Täpänemenach anamihäu "koapenagone, enoch kon aianinichsit; "anan au wechtau Täpänemenach Weau "O Mächkom miniu. Au nanakatawä"nechso änamihat, enechpech tä au meze "anino pachkishigon, au minua miniu " enech ätik anamihäu koapenagon. We" nach maioazin anino, mänik miniu ene, "kon aianinichsit, mitchua minuach miniu "tepenau o panatesin, kon kä o mena"zechtonon ene Täpänemenach Weau."

\section{(1 Cor. xi, 27.-29.)}

Kätin iachpez maze csechzekäu, wätachpenach Jesus Weau as mäk kikäskach mäz päzeton. Isis maze totawen Jesuson, kaiesis maze totawazin enoch kaies käz maze pämatesit inäniu Sudas Iskaiot, kaies tepahakat Täpänechzekänit asau näneme.

Kätau otachpenachkin käz Sawänechtakosin, kon pos o mitchichsinon, kon pochz 
mänawäz, kon miniu pos o mänenon, lies ani apächtxu wanitipächkau. Kespin tä kes kochtamon käkoch, kon pos kit otachpenanon Jesus Weau. Ene neu miniu waiesakesit weiak, kon pos o mitchichsinon, kon miniu pos o mänenon mänawaz nepeu, konemau miniu maskichkiu wapo, echpe kätau otachpenachkin Käz Eucharistiwin. Ene nesik kätau ani pematesit wepoz, ene nesik pos mitchichsit, pos mänik miniu konomächtchiu as otachpenazin Täpänechzekänit Käz Eucharistiwine äpenit.

Mächkawänechtach Jesus o kotakechton o näpin miniu wätachpenamonin Jesus Weau. Ene kaies io esechzekatäk Käz Eucharistiwin, achpäniu asau mächkawänemake kaiesis kotakechtok Täpänechzekät kinach io. Jesus tä iom kes enäu o kächkenohamakanon iskoaz as kes wechpomazin : "Iom kinau esetamuau achpäniu "asau mächkawänemeäk." Käz Paul tä hewa: "Tachnäno kätau mikin enoch " pachkishigon, mänäiäkin miniu enech " ätik anamihäu koapenagon, kinau wech- 


\section{6}

" tamawikin as kes nepäk Täpänemenach, "nanas asau iach piat." (1 Cor. xi, 26.) Ene au esetaion tachnäno wätachpenamonin käz Eucharistiwin.

Zenopomin anamihäwachtik, konau miniu änänechtach: Kätin ne käz tapanik Täpänechzekät. Nenach io kes kipotchiso mächki; nenach io kes säsewahau, nạnas mawau weau kes käz mächkoweu. Nenach io kes pasächkipenau minichsakächsion; nenach io kes pachpenolawau, kes tatachkiskawau, kes papakamau miniu; nenach io kes sachsakachkohau anamilıä wachtiko, nianon tachnäno kes enakanamau, näniu tepachigon nenach io kes akozin anamihäwachtiko, nanas as kes nepäk. Nanakatawänechtach mawau ene wäwänin, opäsik tä kinau kächkenom kaiesis tapane Jesus, opäsik kinau tapanau kinach miniu.

Anamibanon wäwänin katäu otachpenamon Jesus Weau. Kon mänawaz kinau anamihanon, iachpez mäse kinau anamiham. 
Wäwänin tänänemin Jesus käz Ostiwine äsis Hawätokewit äsis mamazetawit miniu. Kon rekotoch nasop osenon Täpänechzekät. Wenach kes hewa: "Iom Neau." "Aiom Ne Mächkom."

Achpäniminon Jesus. Ki katäu menik iochpe tepenau Weau, konau tä ki katäu menik eneko mänäseon äsis otätchiakion, kwaiachk asau pemateseon, asau mächno anochkitawat Hawä tok omos achkihe, ene tä kakik asau mächnion kesekoch. Ene kaies eta Jesus: "Hawänetok mitchichsit ne metchemechsin, mänik miniu ne mächkom, kanawechtau kakik pematesin, nenau iach pematesehau iskoaz kesekach." (Joan. vi, 55.)

Jachpez tapasin Täpäneme tapane. Wawepoz eta iachpez änekochkitähäion: "Jesus käshiateseon, ki tapanin, iachpez neu ki tapanin, änckochkitähäion ki tapanin. Sawänemena, opäsik opäsik asau tapanenon.

A ninichsinon as kes nächkehäion Jesus kaies käz tapane, kaies käz sawäneme. 


\section{8}

Ki katäu wichkoazichtoasim kakik anamachkiach asau wenosion, wenach tä, ki Hawätokom ki kes kisehamach ki päzetonon, ächpez sawäneme.

Pakichsenemin asau moskawänichtamihe, achpäniu waieskewach mihikon kesekoch ächpanik asau pemochneon. Natotamowin asau penetähäon, nawaneu esekin, sochkänichtamoan, kwaiachk pematesin, mawau miniu sawänechzekanon, encko mänäseon, wäwänin asau anochkitawat Hawätok, ene tä kesekoch ision.

Kesänech1ä kon nokotoch asau nächkoseon, asau witchiwat pächsikoaz pämatesit, iachpez miniu wäwänin asau kanawehesion kon nekotoch asau menäion, kon nekotoch asau pächsikoateseon miniu.

Enó tä kon asau nänochtomazin masenachigon, naskat kächkenom Täpänechzekänit, pos anamihäu tä wäwänin äsis wichkichtok. Jachpez mächno eseta akemazin miniu anamihämigon, konau tä mächkawänemäu Jesus o kotakechton; wawänin pos enänemäu Täpänechzekänit 
kaiesis sawänemekot; au tapanäu, au nanakatawänechtäu miniu asau menekot penetähäu kwaiachk pematesin miniu.

Kespin katäu sawänechtakoseon posächkach paienachkin otinon ki machkäsinon tä miniu.

Ene 1ä kespin saiä katäu otachpenamon Jesus Weau, sinawaneu pis nasächlkach käz atuchpon. Mächkotächkoniäu tä pis kochkehat, tachkonach tä onächke käz Ustiwin, enechpe nänino pakachsinon kichpaniach, iom tä eta: "Täpänemeon kon net achpechtänetakosinon, os pos pichtikäion nean, nekot kikitwon tä nesik eta, ene asau inänit nitätchiak."

Kespin tä otachpenamon kitone käz Sawänechtakosin, wäwänin otachpenach, sinawaneu kochtach. Nipitach tä, ishianon atotapiakone. Ene tä wäwänin asau mamiawamat Jesus, kaiesis sawäneme. Kon ene tä kes ponetamakach anamihäwigamiko, pon wepoz kenach sakesenon, kon nawenau kinau sakesim. Kon mänawaz kinau anamihanon, iachpez mäse kinau anamiham, ene tä Josus ki tapanik. 
Anamihanon ätamä konomächtchiu ssau otachpenamä,

\section{KÄZ EUUHARISTIWIN.}

Jesus Täpänemeon, ano kon ki nianenäron, wächzetau kit ächtanänemin Käz Eucharistiwine, ene wä menazehenon, nekoneneu äsis tapanenon.

Jesus Hawätok aiaweion, mawau täpänechtamon, kon netäp achpechıänetakosenon os pos pichtikäion nitähe, pächki net achpechtänetakosim.

Wächzetau ki tapanin, Jesus, waieskesit Nochne nekoneneu; mawau miniu pämatesitoa ne tapanawak, ano as kächkenowapamenon; kon weiak ne sechkanon.

Jesus, kon neiäp achpechtänetakosenon os pos otachpenamọn Käz Eucharistiwine äpeon, mächkawänechtamonin tä ki sawänechzekon, ki pakichsenemin, Jesus neat, asau waieskewechtawon netähe, asau mächno otachpenamon.

Jesus, wächzetau kätin net aninichsim as kes päzetaion; cre wä aninichsion iachpez as tapanckäion; ene wä koskä- 
nechtamon as kes jäzetaion, mächkawänechtamonin ki käshiatesiwin. Kon misik ki katäu nächkehanonon; naszeau opäsik wepoz nepäion konomächtchiu asau nächkehenon.

Pianon, waieskeseon Jesus, pianon, pis tepänechtach netähe. Wächzctau ne monawänechton os pos äpeon nitähe; wächzetau ki natawänechtakosim netähe. Pichtigenon iochpe netähe, Jesus neat; mos* kinatoch netäh ki sawänechzekon. Amen.

Jesus Nochne, nähe sawänechzekäon! Ki kes etan: "Nenau pematesim pach" kishigon, kesekoch ne wächpiam; kespin "weiak muazin anum pachkishigon, au "kakik pemateseu; eno 1ä pachkishigon, "au mikion, ene ne metchemechsim, au "ioch pämatesewak mamazetawak." Ki pis nasärhkon 1ä iochpe, Jesus, asau otachpenamon Keau, asau sawänechtakoseon.

Kon wenach ano netäp achpechtänetakosenon os pos otachpenamon; kinä tä, Jesus, ki natomim ächpez keshiateseon; 
ene wä nasächkonon. Achpez mamachkatänichtakoa Käz Ostiwin, ene ächpez maınachkatänichtakoa ki käshiateseor, kon nekotoch pos netäp nenochtonon.

Kespin naiakatawänichtamonin äsis maze esekion, ne kächkenon, kon käkoch as achpechtänetakoseon, $n$ : kächkenon as kes wichkoazichtoasion anamachkiach ka kik iskotia asau tanächnaseon. Kinä tä, Jesus Nochne, ki iachpez sawäncmim, ki ponikitätawim eneko kaies nächkehenon, ki natomim tä miniu iochpə asau nasächkc,mon asau otachpenamon Keau. Kätin mamachkatänichtakwot ki käshiatesin, Jesus Nochne! Kespin nena mänawaz nekoz achpech1änetakoseon os pos tapanenon, kon pos ne mamachkatänechtanon ächpez mächno totaweon; ne liächkinasim tä kat as täp achpechtänetakoseon, ene wä mamachkatänechtamon.

Jesus Nochne, ki katäu pichtikawin iochpe netähe, kätin wäwänin kit änänemin, ne käz mininichton asau pichtikäon Jesus Nochne. Konäu 1ä ki kohnin, osam 
kä ne maze esekesin; ene tä iachpez wä pakichsenemenon, asau kisehamaweon ne päzetonon, asau penechtawon tä miniu nctäh, asau wichkichtawon wäwänin asau otachpenamon. Amen.

Kes ani otachpenamächkin Käz Ostiwin mamiawanin Jesus.

Ki käz sawänechtakosim iochpe, kit apetak kä Jesus, ki Mäz Hawätokon, Jesus täpanc, kitähe kätin asiu. Pachkishigon äsenakosit ki kes otachpenom Jesus Weau, kinä kaies ioch akozin anamihäwachtiko, ki kes otachpenom kä miniu Jesus O Mächkom, kinä kuies io sekenach Täpänechzekät. Jesus apetam kitäh, ki käkiskawau Täpänechzekät Mäz Hawätok! Kon mamazetau, kon asäniu ki kes pis isitakonon; wenäch neu tepenau Mäz Hawätok mesas käkoch kaies kesechtok, wäkimachkatawazin ıniniu mawau mamazetawon misik tä asäniwon, wenach $\mathrm{Mäz}$ Hawätok kitähe kes pichtikäu, ene iochpe asit. Nawanen apenon mamachkatänechtach ene aiachpez käz achpichı̈̈uichtakoa 
Sawänechtakosin. Kätin mamachkatänichtakwot Käz Ostiwin! Ano nachänit Käz Ostiwin, ene tä asit Täpänechzekät äsis Mäz Hawätok, misik, tä äsis mamazetawit. Äsis mamachkatänichtakoa Käz Ostiwin, ene äsis mamachkatänichtakosit Jesus äsis keshiatesit, äsis tapanazin miniu mamazctawon. Achsamäu änamihanit tepenau Wcau, menäu tä miniu tepenau $O$ Mächkom. Kon weiak nekoneneu aiawit metämoch ot äsis tapananon onitchionechson äsis tapane Jesus. Ene mamiawamin. Anamihanon ätamä kaies ani otachpenamäkin Käz Eucharistiwin.

Jesus Täpänemeon, äpeon Käz Eucharistiwine äsis Hawätokeon misik tä äsis mamazetaweon, nenach io. Änekochkitähäion ki menazehin; kinach liä kes kesechtawon mawau enekoch äsis tanamon, ene wä menazchenon.

Jesus, waieskesit Nochne, ki mamiawamin as kes pichtikaweon netähe; ki tapanin änekochkitähäion; kon nekotoch nenau wanäncchtonon äs mächno totaweon. 
Jesus, täpänechtamon ne pematesin, ki pakitänemon neau misik tă nitätchiak; tepänechtach au esekeon miniu enekoch au kotakechtawon, kesekoch asau mächnoion.

Jesus Täpänemeon, wächzetau kätin ki tapanekosin, wächzetau ki tapanin änekochkitähäon. Sawänemena, sakächnosä netähe, opäsik opäsik asau tapanenon.

Jesus, ki natotamon mächno esekin, ki sawänechzekon mirin; wetochkawena kon nekotoch asau wanänechtonon äsis anamihaion kinach nesik asau kächkenowapamenon.

Wächzetau ne paketäneman maze hawälok; wächzetau ne sechkaton ne maze esekin; kon nekotoch ne katäu kächkenowapachtonon mätä, kinach nesik, Hawätok Nochne, ki katäu kächkenowapamin. Sawänemena. Amen.

Jesus sawänemeach! Jesus sawänomeach! Jesus sawänemeach!

Käz Marie, Jesus Okiän waieskeseon, keketotamoweach! 
Käz Joseph, neat käz tatachkeseon, natamoweach!

Käz Mishän, okemau asäniwon, natamoweach!

Mawau neu kesekoch ächtanachkiek, nazekapawetochkon Hawätok, asau keketotamoweach. Amen.

Misik tä pos eta nekotono: "Nochnenau kesekoch äpeon," "Kit anomächkon, Mari," "Ne papamächtawau," "Käz achpechtänetakosit Wäwochneme." "Mächkawänechtach, käz Mari,"

Iskoaz tä eta nekotoasita ene: "Nochnenau kesekoch äpeon," “Kit anomächkon, Marie," "Käz achpechtänetakosit Wäwochneme." Ene tä :

"Käz Otä Jesus natamowena opäsik opäsik miniu asau tapanenon." Amen.

Mamiachtozekan Jesus.

Täpänemeon, sawänemeach.

Jesus Christ, sawänemeach.

Täpänemeon, sawänemeach.

Jesus Christ, pächtaweach.

Jesus Christ, sawänochtäweach. 
Mäz Hawätok, Wäwóchnemekäon kesekoch äpeon,

Mäz Hawätok, Wäkisemekäon kaiés akuasemàtoa pämàtesitoa,

Mäz Hawätok, Wieskeséon Hawätok,

Mäz Hawätok, as Nänión Nekónenen as áweon,

Jesus, Mäz Hawätokeon,

Jesus, Mäz Hawätok Wägiseme,

Jesus, kaiäz achpechteséon, Kóchne Mäz

Hawätok äsis käz achpechtesit,

Jesus, käz Mari Wägíseme,

Jesus, maioskinäskakoian mawau neu mächno esekin,

Jesus, mamàwau maiamàchkatänemekäon,

Jesus, kàkik pämàteseon,

Jesus, mesas käkoch nähechtawon,

Jesus, aiachpez keshíateseon,

Jesus, täpänechtamon ne pämatesininawon,

Jesus, täpanatoa nitätchiakonawak,

Jesus, nähe - sawänemeach,

Jesus, kaiäz achpechtänetakoseon,

Jesus, maiächno kanawihatoa nitätchiakonawak, 
Jesus, wänanekosehatoa asäniwak, Jesus, saiochkäníchtamihatoa anámihan wächzinánichtoa,

Jesus, waiáchsinakihámawatoa kwaiàchk äsichzikätoa kit äséchzekon, Jesus, aiachpez nawachneu esekeon, Jesus, täpachnenichsion änekochkitähäon,

Jesus, täpatamon nipuachkon esekin, Jesus, täpatamon nawachneu esekin, Jesus, täpiseach,

Jesus, aiachpez tapanekäon,

Jesus, täpanatoa kätemakesitoa,

Jesus, kächkenohamawatoa änonatoa,

Jesus, kaies pis mamazetawihision, as

kes pis pemazeheach,

Jesus, kaies pis ochtateseon kitämakegine, as kes pis sawänemeach,

Jesus, kaies pemateseon nänino metátach misik näniu pepon ios achkihe, as kes kächkenohamaweach,

Jesus, kaies käz kotakechtawon as kes mál pemateseon achkihe,

Jesus, kaies kipotchision mächki, as kes käz anemeseon konomächtchiu as tachkonikäor, 
Jesus, kaies sachsakachkohokäon anamikääàchtiko, nénach io,

Jesus, kaies käz wéchsakänechtamon anamihä wachtiko,

Jesus, kaiès tachpänäon anamihäwach-

tiko as kes pemazchatoa nitätchiakonawak,

Jesus, kaies apiskaion,

Jesus, kesekoch kaiés ision,

Jesus, wäkemaweon kesekoch mesas tä miniu achkihe,

Jesus, au tipachkonatoa mawau neu pämatesitoa,

Hawätok o manistanechseman, käsihamawatoa pämatesitoa o päzctoniwau; Ponikitätaweach.

Hawätok o manistanechseman, käsihamawatoa pämatesitoa o päzétoníwau; Sawänochtaweach.

Hawätok o manistanechseman, käsihama. watoa pämatesitor o päzetoniwau; $S \mathfrak{r}$ wänemeach.

Jesus Christ, pächtaweach. Jesus Christ, sawänochtaweach. 


\section{0}

Täpänemeon, sawänémeach. Jesus Christ, sawänemeach. Täpänemeon, sawänemeach. "Nochnenau," “Kit anomächkon," “Käz achpechtänetakosit."

Jésus, Márie, Jóseph,

Ki pakitänamon

Neau, netäh, netätchiak,

Ne pämatesin miniu. Amen.

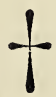




\section{K $\dddot{Z Z ~ P A K E T E N E K O N . ~}$ Kakichkotakan.}

Tachnäno Anamihäkesekach, tachnäno miniu Käz Kesekach, kinau isiam as tase anamiha anamihäwigamiko, wäwänin miniu kinau anamiham as mäk Mäz Anamihan. Ene kaies enachkonikätoa ochkano Käz Mächkotächkoniäwak, ene äsitatoa miniu mawau kwaiachk änamihatoa mesewäu änekochkoa achkäu. Eno tä kon kätau isiat as tase anamiha Anamihäkesekachkin, Käz Kesekachkin miniu, kätin käz maze esetau, käz nächkehäu Mäz Hawätokon, kon as katäu pächtawazin mänätamechkenit anamihäwigamiko, eno kä Käz Mächkotächkoniäwon. Iom kes hewa Jesus: "Hawänetok kon au pächtawazin anamihäwigamiko, naskat pos achpechtänemau, kon änamihat, käz päzetau miniu ächpechtäneme." (Matt. xviii, 17.) Ene aio kesänechtamon kon nekotoch asau wanechnetawon Mäz Anamihan Anamihäkesekachkin, Käz Kesekachkin mi- 
132

niu. Jachpez weskiwat miniu änochkikesekachkin asau ision as tase anamiha. Sawänechtakoseu änamihat tachnäno kesegot isiat! Kespin tä kat wichkichtok tachnäno kesegot anamihäwigamiko asau ision enekoch äsis wichkichtawon isianon. Jesus tä käz mächno echpänonik kesekoch.

Wäwänin anamihanon as mäk anamihat Mächkotächkoniäu. Zenopachtach Mäz Anamihan, anoz miniu anamihanon kinau wechtamon. Jachpez weskiwat miniu asau akimatoa anamihämiguk, as mäk Mäz Anamihan. Mämik tä akemin anamihämiguk, kespin kon nenämon masenachigon. Akemin tä iachpez änekochkitähäon wäwänin, konau nanakatawänechtach Jesus o kotakechton o näpin miniu.

Pon kaiänit inänechtach as mäk anamihat Mächkotächkoniäu, ene nesik zenopachtaćh Mäz Anamihan, papik tä paketach kaiänit inänechtamon.

Pon anapenon mesewäu anamihäwigamiko; pon zenopomin enis äpitoa; pon keketotawin miniu. 
Pon aiänenon; pon miniu anapenon iskoachtäme, enoch pos pichtiket weiak. Pon akuanämin nänemau. Pon sachkoazekenon anachkichzekone. Pon mianazetach. Pon tatakazichkinon. Io menänach ki nächkon kipaniach asau anamihaion. Otchichtchikoanetach Mächkotächkoncäu as otchichtchikoanetat.

Mächkawänemin Jesus, ki zenopomik esetaion anamihäwigamiko. Achpäniu pis isianon asau konomächtchiu wäp anamiha, pon nekotoch sakesenon anamihäwigamiko, konomächtchiu asau achpechtänechtamon Mäz Anamihan. Opäsik asau achpechıänechtamon Mäz Anamihan, achpäniu wäwänin asau anamihaion as mäk Mäz Anamihan, nanakatawänechtach äsetot Mächkotächkoniäu as mäk anamihat.

Echpe paiazin as tase anamihat, anamihäwachtikonikäu, anamihäu tä, aninichswon miniu wechtam.

Nawenau tä na-ächkon anamihäu masenachigon, ene tà as anamihat. Hewa "Gloria in excelsis Deo," asau käz mena- 


\section{4}

zehazin Hawätokon, kaiesis käz menazehatoa Asäniwak Nipatejä Anamihäkesekach. Misık tä anamihäu wechtam miniu ochkano kaies isichziketoa kaies mächno kächkinachkoa inäniwak misik tä Jesus o kächkenohamakanon.

Nawenau tä enoch änochkitawazin Mächkotächkoniäwon iaziatau masenachigon, ene tä Mächkotächkoniäu as wechtach Jesus o kikitoanon. Enechpe mawau anamilı̈̈wigamiko aitoa nipitawak, ene tä as anamihäwachtikoniketoa ochkächnoa, otonoa, misik ochpaniachkowa.

Misik*tä Mächkotächkoniäu nawenan pis nepaweu, ene tä as wechtach "Credo," "Ne papamächtawau Hawätok Wäwochneme" . . Nawenau paketenamowäu Hawätokon pachkishigonon somenapo miniu, kisechnetan onächgon miniu, konau tä anamihäu.

Misik käz menazehäu Hawätokon, ene tä as wechtach: "Sanctus, Sanctus, Sanctus," ene kä, "Käz achpechtïnetakoseu Tïpänemenach Hawätok." Äsis käz menazehatoa Hawätokon asäniwak kaiäz achpich- 
tänichtakositoa kesekoch äpitoa, ene tepa miniu wenach Mächkotächkoniäu äsis käz menazehazın enechpe Täpänechzekänit.

Nawenau tä keketotamowäu mamawau mänätamechkenit Käz Mächkotächkoniäwon, mawau miniu anamahä wigamiko mänätamechkenit.

Misik tä mächkawänemäu, anamihätawäu miniu mawau Katolik änamihanit änekochkoa achkäu; mämik keketotamowäu aninoch, wäch anamiha enechpe, mawau miniu anamihäwigamiko äpenit anamihätawäu.

Kes ani keketotamawikit tä, tepach esetau Mächkotächkoniäu, kaies esetot Jısus, as kes osechtok käz Sawänechtakrisin (Eucharistiwin). Otachpenäu pachkishigon onächke, anamihätawäu, ene tä as wechtach, kaies eta Jesus: "Iom Neau." Nawenau tä otachpenam anamihäu koapenagon, anamihätom tä somenapo, ene tä as wechtach, Jesus kaies eta, "Iom $\mathrm{Ne}$ Mächkom." Ispänom käz Ostiwin, ene kä, Jesus Weau pachkishigon ot äsenakosin; ispänom miniu anamihäu koapenagon, 
ene ätek tä Jesus O Mächkom, somenapo ot äsenakosin. Ene tä ene wä esetot, mawau änamihatoa anamihäwigamiko äpitoa asau menazehatoa Jesuson anamihäu atuchpone äpenit.

Misik tä anamihäu Mächkotächkoniäu, konau tä mächkawänechton Jesus o kotakechton misik tä o iachpematesin. Nawenau tä keketotamowäu mawau änamihanit kaies näpenik, kisichnasoane kotakechtonik, Hawätokon asau sawänemekotoa, wepoz asau otachpinikotoa kesekoch. Mämik tä anamihätawäu anino kaies näpenik, wäch anamihat enechpe. Kes ani keketotamowazin kisichnasoane äpenit, wechtam ene anamiban: "Nochnenau kesekoch äpeon." Jesus tepenau kikes kächkenohamakonau ene anamihan, ene wä mänätamechkemakach.

Misik tä anamihäu Mächkotächkoniäu, nänino pakachso ochpaniach konau pakichsenemäu Hawätokon, asau sawänemekot. Nawenau tä pakichsenemäu Jesnson, asau wetochkakot, wäwänin asan otachpenach käz Sawänechtakosin(Eucha- 
ristiwín). Misik nänino paka(hso ochpaniach, ene tä as wechtach: "Täpänemeon, kon netäp achpechtäsinon, asau pichtikaweon, nekot kikitwon nesik wechtach, ene asau inänit nitätchiak." Konau $1 a ̈$ Mächkotächkoniäu otachpenom Jesus Weaı o Mächkom miniu.

Kespin tä anech änamihatua katäu olachpinachkoa käz Sawänechtakosin, enechpe wätachpinachkoa. Kes ani otachpinamä Jesus Weau, anamihäu Mächkotächkoniäu. Mamiawamäu Jesuson, as kes menekot käz Eucharistiwin, anamihätawäu miniu mawau anamihäw gamiko äpenit. Iskoaz tä wechtam kaies eta käz Joan Jesus o kächkenohamakanon, ene tä as ponetot.

Opäsik wäwänin achpechtänechtamon käz Anamihan, nanakatawänechtach äsikimakach. Paketenekon kä. Pis äz achkewik, akiko kaies anochkitawatoa Hawätokon, kes nächno paketenekäwak. As kes pis isiat omos achkihe Jesus, kes paketänechso, as kes tachpänät anamihäwachtiko. Echpe kaies jaketenamuazin Weau 


\section{8}

O Mächkom miniu Hawätokon, nänino waichsinit, ene tä enech iachpez kaiäz achpichtänichtakoa paketenekon. Kinach tä kes io paketänechso Jesus, Hawätok asau kisehamonach ki päzetonenawon misik asau pachkinikatäk kesik.

Achpäniu tä asau katäu mächkawänechtamach ene paketenekon anamihäwachtiko, Jesus kes osechtau käz Anamihan, iskoaz as kes wechpomazin o kächkenohamakanon. Kes otachpenom pachkishigon onächke, kes anamihätom, kes menäu tä anino inäniwon jom kes enäu: "Otachpenamokon, metchekon miniu, Iom Neau." Koneu tä somenapo kes otachpenom anamihäu koapenagon, kes anamihätom, kes menäu o kächkenohamakanon, iom kes enäu: Menäkon, kinua mawau, Iom N'e Mächkom. Iom miniu kinua au esetaiäk, kakik asau mächkawänemeäk." Kätin pachkishigon otäsenakosin kes atau Weau, kes hewa: "Iom Neau." Somenapo tä otäsenakosin kes atau O Mächkom, kes hewa: "Iom Ne Mächkom." Ene tä echpe Jesus kes osechtau ene iachpez kaiäz achpich- 
tänichtakoa paketenekon, Mäz Anamiban äs wechtek.

Kon nisinon anamihäwachtik paketenekon; Anamihäu paketenekon miniu; nekoneneu nesik, ene tatepach achpichtänichtakoa. Kaiesis paketenesit Jesus anamihäwachtiko, ene tepa äs paketänichsit anamihäu atuchpone tachnäno änamihazin Mächkotächkoniäu. Kon tä o näpenon Jesus echpe Mäz Anamihan, kaiesis nepäk anamihäwachtiko, kon misik o sekinanon O Mächkom. Kespin mawau ene wäwänin nanakatawänechtamon kinau nänochton achpichtänichtakoà Mäz Anamihan. Kon käkoch omos achkihe isis achpichtänichtakonon, Mäz Anamihan äsis ächpichtänichtakoa.

Aiatchkwot tä nanakat:lwänechtach, wäki aiawik Mäz Paketenekon. Ene tä:

1. Arhpäniu asau mächkawänechtamä Jesus, kaiesis kotakechtok, kinach io, as kes päketänichsit anamihäwachtiko.

2. Asau käz achpechtänemake Hawätok, asau menazehakech miniu. 
3. Asau mamiawamake Hawätok as kes käz tapanenach, as kes käz mächno echpänanenach miniu.

4. Asau sawänemenach Hawätok, asau menenach kwaiachk aninichswon ene asau kisehamonach ki päzetonenawon.

5. Asau menenach mawau käkoch mänäseach äsis oweawe, äsis otätchiakiach miniu.

6. Asau menazehake kaiekachkoit käz Marie, mawau miniu kaiäz achpichtänichtakositoa kesekoch äpitoa asau menazehakichtoa.

7. Asau sawänemakichtca kisichnasone kotakichtokoa, mämik tä kochnenau kikiachsenau miniu.

Nanakatawänechtach mawau ene wäwänin, kesänechtach archpaniu asau käz achpechıänechtamon Mäz Anamihan ächpez anamiha.

\section{KÄZ PAKETENEKON. \\ Mäz Anamihan, äs weihtek.} Anamihan ätamä paichtikächkin anamihäwigamiko, as otachpenamä tä anamihäwapo. 
Hawätok Tapänemeon, pinichtowena netäh, netätchiak miniu, asau mininichtamihinon omos achkihe, kes nepäion tä, kesekoch asau ision.

Kotamikwot, ene omos Hawätok wek, io miniu kesekoch iskoachtem ätamä, Hawätok wek miniu, Hawätok mesas käkoch nähechtawon! wächzetau kätin osam mäninechtom nitätchiak, Täpänemenach wek ne katäu isiam. Amen.

\section{ASPERGES.}

Säsopinamawena, Täpänemeon, nenau penesim, kiseialpawasena, opäsik tä kohn äsis wapiskenit nenau isis wapiskenim. Sawänemena, Hawätok, kinä nesik sawänechzekäon.

Käz achpechtänetakosit Wäwochneme etc.

Anamihan ätamä konomächtchiu as wäp Anamiha.

Hawätok, Nochne, sawänemena, wäwänin asau anamihaion as mäk Mäz Paketenekon, wäwänin asau menazehenon, 


\section{2}

wäwänin asau mamiawamenon eneko rnaiächno echpänaseon. Sawänemena, asau aninichsion, asau io kisehamaweon ne päz esetanon, äsis achpänimion Kikis Jesus o kotakechton. Amen.

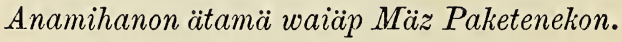

Hawätok Nochne, ne käz päzetom, kon pochz netäp achpechtänetakosinon asau anapeon kesekoch, kon miniu pochz asau zenapachtonon as tase anamiha, asau menazehenon; ne pakichsenemawak tä kaiäz achpichtänichtakositoa kesekoch äpitoa, asau keketotamawitoa. Ki mamiachtomin, Hawätok Nochne, asau ponikitätaweon, asau meseon ki sawänechzekon. Sawänemena, kisehamawena, ne maze esekin, Jesus o wichswon io kisehamawena.

Ne wechtamowau Hawätok mesas käkoch nähechtok; etc.

Naszeau kat ki sawänemim Hawätok mesas käkoch nähechtok, asau kisehamonach ki päzetonenawon, ene tä asau ishiaseach kakik pematesine. Amen. 


\section{GLORIA.}

Käz achpechtänetakoseu ispämiach Hawätok, wachketachkamik tä wanachkiwak pämatesitoa maiächno inänichtachkoa. Ki mächno wechnekem. Kit anamihätakem. Ki käz monazehekäm. Ki mamiawamekem ächpez käz achpechtänetakoseon, 'Iäpänechzekäon, Hawätokeon kesekoch wäkimaweon, Wäwochnemekäon mesas käkoch nähechtawon. Täpänechzekäon Jesus Christ, Hawätok Wägisemckäon nekoneneu aiaweon, Täpänemenach Hawätok, kaies paketänechtamon keau; kinach käsihamowatoa pämatesitoa o päzetonenowawon, otachpenamoweamä äsis ınamiachtomekäon. Kinach mäsächsenon Wäwochneme otäniniane, sawänemeamä. Kinä nesik kaiäz achpechtänetakoseon; kinä nesik Täpänechzekäon; kinä nesik iachpez ispänechtakoseon; kinach Jesus Christ miniu Waieskesit Hawätok, Mäz Hawätok Wäwochneme o käz achpechtänetakosene. Amen. 


\section{4}

Echpe Mächkotächkoniäu näkamizin kakichkotakan. (Epistle.)

Hawätok Täpänemeon, naszeau kat ki mesim ki sawänechzekon, änekochkitähäon asau tapanenon; äsis tapanision asau isis tapanakoa nez pematesewak. Sawänemena, kisehamowena kaiesis päzetaion; sochkitähäskawena asau päz esechzekäon Jesus ot anamihan omos achkihe, kes ne'päion tä kesekoch asau ision.

Hawätok Täpänemeon, kikes kächkenohamowak pämatesitoa, Jesus konomäshtchiu as pis ochtatesit achkihe, menä kächkenomuk inäniwak, akiwak kaies anonatoa; echpech tä Kikis Jesus kaies piat achkihe, wenach ni kes kächkenohamakonau kit anamihan; kaies ani pematesit Jesus achkihe, o kächkenohamakonon kes pimiu kächkenohamawikowon kit anamihan änekochkoa achkäu.

Sawänemena, Jesus, wasinakichtowena, kwaiachk asau kächkenamon kit anamihan, mawau miniu asau onamichtamon, wäwänin miniu asau esetaion ene kä kaiesis kächkenohamawikion, ki kächke- 
nohamakanok miniu kaiesis kakichkimiwätoa. Mämik tä sawänemena kon nekotoch asau wanänechtamon ene kakichkotakan: "Ene kä wä kächkenamon Jesus o käshiatesin, as kes paketänechtach o pematesin kinach io; ene tepa miniu kina pos ki paketänechtä ki pämatesininawon kez pämatesinawak io.

Kespin weiak kächkatesit omos achkihe, näwäzin tä wez pematesewon kätemakesinit, kon tä sawänemazin, tane pos äsis tapanazin Hawätokon? Pon ene kat kit äseke, asau eiach nesik: ne tapanau nez pemateseu; kätin kä kat ki tapanonau, kat ki mächno echpänonau."

\section{Echpe Naipitachkin.}

Ene iochpe as zenapachtach Mächkotächkoniäu Jesus o kakichkotakan. Iachpez ne kesänechton kwaiachk asau esetaion mawau eneko Jesus kaiesis kächkenohamonach, Wenä nesik Jesus kaies eta: "Hawänetok au onamichtach, au esetot miniu net anamihan, saikahapuane tä miniu, eno tä kesekoch au isıat." Opäsik pos ne 
mäninechton asau nächnekäon, ene tä asau nekatamon Jesus ot anamihan.

Jesus, Hawätok Wägiseme, ki kes pis isiam omos achkihe as kes pis kächkenohamaweach kwaiachk Hawätok ot anamihan; Sawänemena, mesena ki sawänechzekon, kwaiachk asau kächkenamon kaiesis kächkenohamawikion, wäwänin miniu asau esechzekäın kit anamihan au $\ddot{a} z$ pemateseon. Amen.

\section{CREDO.}

Ne papamächtawau Hawätok Wäwochneme, etc.

Anamihan ätamä, Mächkotächkoniäu paketänamowazin Hawätokon pachkishigon, somenapo miniu.

Hawätok Täpänemeon, Mächkotächkoniäu iochpe ki paketänamak pachkishigon somenapo miniu; nenach tä ki paketänamon neau, netätchiak miniu. Hawätok Nochne, kinach nachäs tepänenach; eneko au enänemeon ene au esekeon.

Sawänemena, iachpez kisehamawena ne maze esekin: iachpez wanänechtamawena 
mawau ne päzetonon. Iazichtowena netäh asau minitähäion, asau kon nekotoch kikäskamon netähe käkoch mäte. Amen. Echpe Mächkotächkoniäu käsichninätchichsinonächke.

Hawätok Täpänemeon, kisenemin nitätchiak asau penesit, asan kon kikäskach päz esekin. Hawätok Nochne, isis paketenamakäon, naszeau kat ki mänazehckäm. Kit äsis pakichsenemin miniu, iom Mäz Paketenekon asau isis paketenamakäon, asau io inänit nitätchiak, mawau miniu änamihatoa asau mächno enapazichtokoa. Amen.

Echpe Mächkotächkoniäu anamihazin "Orate Fratres."

Hawätok Nochne, kakik kanawehena net ächpez kitämakesim, kor nena tepenau pos ne wichkichtoasinon asau mächno esckeon. Ki pakichsenemin tä, Jesus o wichswone ki io pakichsenemin, asau sawänemeon, achpäniu asau mächkawänemenon, opäsik asau kächkenomon, nenach tä miniu wäwänin asau kächkinaseon. 


\section{8}

Kespin wäwänin kächkenomon, opäsik kinau tapanin. Kespin tä wäwänin kächkinaseon, opäsik nenıu tapachnenichsim. Sawänemena asau kächkinaseon, asau waninäwänichsion, kon nekotoch miniu asau wanäuänechtamon kaies eta Kikis Jesus: "Enoch au ispänechtach weau, au tapanenechtakoseu; ene tä au tapachnenichsit, au ispänechtakoseu." Iachpez paketon, mawau ne maze inänichtamon, mamenatesin miniu. Sawänemena asau tapachnenichsion, kakik miniu asau mächkawänemenon. Amen.

Echpe Mächkotächkoniäu näkamizin: "Per omnia saccula saeculorum."

Hawätok Täpänemeon, mawau asäniwak, kaiäz achpechtänichtakositoa miniu kesekoch äpitoa ki mänazehekok, kon nekotoch o täpäniminowawon as nekamitoa ächpez minatänimächkoa. Nenach tä miniu, aiano käz päz esekeon, tne ätoa asäniwak, kaiäz achpechtänetakositoa miniu, nisem nenach nap: 


\section{SANCTUS.}

Käz achpechtänetakoseu,

Käz achpechtänetakosen,

Käz achpechtänetakosen,

Hawätok Täpänemenach.

Mamachkatänechtakoseu äsikit; menazchek kesekoch asäniwon, misik tä omos achkihe pematesinit.

Ene iochpe keketotamowäu Mächkotächkonï̈u mawau änamihanit änekochkoa achkäu; mämik tä keketotamowäu anino, wä anamihat, mawau miniu anamihäwigamiko äpenit mächkawänemäu keketotamowäu miniu.

Hawätok Täpänemeou, pon echpänasena kaiesis wichkoazichtoaseon as kes päz esetaion; pon miniu säsewahena eneko kaiesis nächkehenon. Täpänemeon, pon mächkawänechtach käios ne maze esekin; wepoz sawänemena, osam ne kätemakesim.

Hawätok Nochne, sawänemin mawau Mächkotächkoniäwak, mawau änawämakoa, mawau maiächno totawitoa, mawau täpasitoa, mawau miniu saichkasitua, mesin mawau ki sawänechzekon. 
Sawänemin mawau änamihatoa änekochkoa achkäu ächtanesitoa. Kit äsis pakichsenemin, Täpänemeon, asau kanawänechtamon Jesus ot anamihan omos achkıhe, kakik wäwänin asau isichziketoa mawau änamihatoa, wäwänin achpäniu asau anochkitochkoa kaiesis kächkenohamaweamä Kikis Jesus; kakik miniu asau wanachkitoa, nekoneneu asau inänichtachkoa, sawänechtakosin anamihan asau tapatachkoa. Amen.

Ene iochpe äsechzekät Mächkotächkoniäu, kaies esechzeket Jesus as kes osechtok

\section{Käz Eucharistiwin.}

"Aiatchkwot as kes wechpomazin o käch"kenohamakanon pachkishigon kes otach"penäu, kes anamihätawäı, kes nänich"towäu tä minin o kächkenohamakanon, “kes enäu :" “Otachpenamokon, mezekon "miniu mawau: Iom kä Neru." Somenapo "kes otachpenam, as atek anamihäu koa"penakone, kes anamiliätam, kes menäu tä 6'miniu o kächkenohamakanon, kes enäu : "Otachpenamokon, menäkon miniu ma- 
"wau: Iom kä Ne Mäclkom, kinua au io "sekechnä, mäse tä miniu pämatesitoa, "asau ponikitätakotoa Hawätokon päzi"tatoawin." (Matt. xxvi.)

Echpe Mächkotächkoniäu äspenachkin Käz Ostiwin, Jesus Weau, Hawätok nekoneu, nikitchkitach, nänino tä pakachsinon kichpaniach, misik tä kinau nänino änekochkitähäon etan :

\section{"Jesus, Täpänemeon, sawänemena."}

Misik tä echpe Mächkotäshkoniäu äspenachkin Käz O Mäshkom anamiläu koupenakone, nikitchkitach, nünino miniu pakachsinon kichpaniach, änekochkitähüon tä kinau etan nänino:

\section{"Jesus, Täpänemeon kischamawena ne päzetonon."}

Kaies ani espänamächkin Jesus Weau

o Mächkom tä miniu.

Jesus Nochne, kina äjeon käz Ostiwine, achpäniu mächkawänichtamihena as kes nदุ|näcltkä on anamihäwachtiko, as kes sekenamon mawau neu ki Mächkom nenach io, as kes pemazehat netätchiak, kakik isko- 


\section{2}

tia os pos kes tanächnasit. Änekochkitähäon ki mamiawamin; net aninichsim mawau neu eneko kaies päzctaion; wächzetau ne kesänechton kat misik asau päzetaion; sawänemena tä miniu sochkitälıäskitwena.

Hawätok Täpänemeon, wächzetau iochpe net aninichsim eneko kaiesis nächkehenon as kes esekeon. Iachpez neu ki käz achpechtänetakosim, iachpez tä miniu ki nähe sawänechzekäm, achpäniu ki. mächno totawin, ene wäsechkatamon mawau neu ne päzetonon.

Kätin sechkatänechtakoaton; kon käkoch ios achkihe net äsis sechkatonon, äsis sechkatomon mawau ne maze esetonon. Kinä, Täpänemeon, wä sechkatamon ne päzetonon, ächpez wieskeseon, ärhpez miniu nähe sawänemeon; soch tä ki maze echpänonin. kon wäwänin ki pächtonenon, ene tä wäch aninichsion, one wä koskänechtamon as kes päz esekeon. Sawäne"mena, kisehamawena, Jesus o wichswon io kisehamawena. Ne k:ı täu mächno kanawihisim, kat misik asau päzetaion. Sawä- 
nemena, wetochkawena; kon nachäs pos ne wichkichtonon kwaiachk asau esekeon. Sochkänichtamihena asau mächno kanawihision. Jesus o wichswon ki pakichsinemin asau meseon sochkänichtamowin, ki sawänechzekon tä miniu.

Jesus nähe sāwänechzekäon, sawänemena, änekochkitähärn asaụ onamichtamon. Ki tapanin, Jesus; sawänemrna, opäsik asau tapanenon. Ne iachpez koskänechton as kes päzetaion, ne nanakatawänechton kaiesis anemeseon ios achkihe, nenach io; ene tä wä koskäncchtamon. Sawänemena, kwaiachk asau esekeon. Ne mächkawänechton, Jesus, kaiesis, kotakechtawon as kes sachsakachkoahokäm anamihäwachtiko, as kes nächnekäon-tä miniu, as pemazehat netätchiak, ene wä rapanenon. Amen.

Ene iochpe as keketotamowazin Mächkotächkoniäu kisichnasoane kotakechtonik; keketotamawin tä miniı kinach ap..

Jesus Täjänemeon, sawänemin mawau neu kisichnasoane äpitoa; mämik ne 
nekehekok, ne nitchionechsok, net ünawemakanok tä miniu. Sawänemin tä miniu kaies mächno echpänasitoa, kaies tapasitoa tä miniu. Kitämakänemin tä miniu kaies sichkasiton, kaies maze echpänasitoa miniu. A kuachsemin äsis kotakänichtakoa; kakik asau wanachkitoa kesckoch asin. Amen.

\section{PATER NOSTER.}

"Nochnenau kesekoch äpeon."

Ene iochpe as anamihat Mächkotäch. koniäu, Hawätok asau miakonamona ma. wau neu mäte.

Hawätok Täpänemeon, zikakiach achpaketowena mawau neu mäte, kaiesis wichkoazichtoasion as kes päzetaion; mämik tä kanawehena kat asau päzetaion kakik iskotia kat asau achpakenekäon.

Jesus Täpänemeon, kaies enänechtamon, nianon neno asau enakanamekäon, as:au nächnekäon miniu anamihäwachtiko, asa u pemazehatoa pämatesitoa, enänemena asau kisehamakäon ne päzetonon.

Sawänemena asau kwaiachk esekeon 
nanas asau nipäon, ene tä kes nipäon kakik asau nämon kit okemawin keses koch. Amen.

\section{AGNUS DEI.}

Hawätok o manistanechseman, käsihamawatoa päzitatoa o maze isitanowawon, sawänemeach.

Hawätok o manistanechseman, käsiha* mawatoa päzitatoa o maze isitanowawon, sawänemeach!

Hawätok o manistanechseman, käsiha mawatoa päzitatoa o maze isitanowawon, wanachkiheiach!

Ene io pos ätamï näpikoa as ioch anamiha.

Hawätok o manistanechseman, käsihamawatoa päzitatoa o maze isitanowawon, asau wanachkitoa asiu!

Hawätok o manistanechseman, käsihamawatoa fäzitatoa o maze isitanowawon, asau wanachkitoa asin!

Hawätok o manistanechseman, käsiha mawatoa päzitatoa o maze isitanowawon, kukik wanachkiwene kesekoch asin! 


\section{DOMINE NON SUM DIGNUS.}

Täpänemeon, kon net achpechtänełakosenon, os pos pichtikäion netähe, nekotono tä eta, ene tä asau inänit netätchiak. (Pos eta nänino.)

Echpe Mächkotächkoniäu wätachpenachkin Jesus Weau O Mächkom miniu. Krspin anäch änamihatoa katüu otachpenachloa Käz Eucharistiwin, ene iochpe asau otachpenachkoa.

Jesus, sawänemena, pinichtowena netäh, wätachpenamonin Käz Eucharistiwin asau mächno otachpenamon. Sochkitähäskawena, kat nekotoch asau panazechtawon ki sawänechzekon, äsis meseon asau mächno otachpenamon Keau.

Jesus, Hawätok Wägisemekäon, ki kes pemazehawak pämatesitoa, tepenau ki pämatesin ki kes paketänechton, as kes papamächtawat Kochne Hawätok; änekochkitähäon ki pakichsenemin, asau pinichtoweon netäh tachnäno wätachpenamonin Keau aiachpez käz achpichtänichtakcı, asau mächno otachpenamon. 
Sochkänichtamihena miniu, achpäniu wäwänin asau kanawechtawon ki kikitwon nanas asau anihion ios achkihe.

Jesus Täpänemeon, sawänemena, kanawehena kat nekotoch asau maze otachpenamon Keau. Jachpez neu kätin käz maze otachpenach. Tepenau o panazechton mitchua, kespin maze otachpenach Keau. Sawänemena, Jesus, achpäniu wäwänin asau otachpenom.

Hawätok Täpänemeon, ki mamiawamin mawau neu kaiesis meseon; mämik ki mamiawamin as kes pis enäsehat Kikis Jesus ios achkihe as kes pis pemazeheamä, kes paketänechso, kes kotakechtau tä miniu, nenach io, asau akuachsemeamä kakik iskotia. Amen.

Echpe Mächkotächkoniäu naiasächkakin anamihäu masenachigon, asau anamihat.

Jesus Nochne, ki iachpez tepänemawak mawau neu pämatesitoa; ki täpänechton pematesin, nepän tä miniu iachpez tepänechton. Wäwänin ki mamiawamin as kes kitämakänematoa mawau pämate- 
sitoa; nenau akuachsemawak, nenau pemazehawak, as kes inänematoa. Kätin ki kes käz kotakechton as kes pis sawänemeach. Wäwänin ki mamiawamin as kes kipotchision Ki Mächkom. Nesechkaton mawau ne maze esekin. Wetochkawena, Jesus, kakik asau papamächtomon. Amen.

Nas tä kakik wanachkione kesekoch kat apewak mawau neu änamihatoa kaies mächno nepäkoa! Amen.

Nas tä Hawätok kat ki menekonau ot anamihäu sawänechzekon! 为 $\mathrm{O}$ Wichswone Wäwochneme misik Wägiseme misik Waieskesit Hawätok! Amen.

Ene iochpe as kakichkotakät Mächkotächkonï̈u kaiesis asachzekät käz Joan, Jesus o kächkenohamawekomon.

Jesus kaies akuachsemeon, kätin Hawätok kit Okisemik; ene neu äsis Hawätokeon, Wäwocheme äsis Hawätokewit, Waieskesit Hawätok miniu äsis Hawätokewit; ki kes pis mamazetawehesim tä, ki kes nächnekämtä miniu anamihäwachtiko, as kes pemazehat netätchiak; iochpe 
kakik ki wekemim Käz Eucharistiwine. Kätin ki käz tapasin. Sawänemena, änekochkitähäon asau tapanenon wäwänin tä asau anochkitonon au äz pemateseon. Amen.

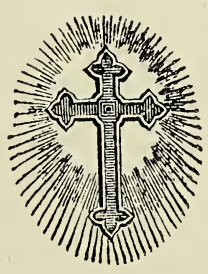




\section{KES ANI NAWACHKIKIN ANAMI- HAN. (VESPERS.)}

Kon ene isis täpechnänon, os pos ishiach as tase anamiha Anamihäkesekachkin Kaiäz Kesekachkin miniu. Kaies ani nawachkikin kinau pichtikem misik anamihäwigamiko, asau anamihaion, asau nikamion miniu. Wäwänin pächta tä miniu anamihäu kakichkotakan, opäsik asau nänochtamon Jesus ot anamihan.

Enechpech tä Mächkotächkoniäu äspenachikin Jesus Weau, iachpez neu änekochkitähäion menazehen Täpänechzekät enis pachkishigone otäsinakosin as apet, pakichsenemin tä miniu asau saweneme miniu. Ki käz mächno esetom asau akimatoa anamihämiguk ächpez nächka as nikami. Mämik nochpeme aianin, akemin anamihämiguk.

Pon tä isianon as tase menä, as tase atati, as tase nimiheti miniu. Mawau neu ene iachpez matät. Kon miniu pis ki pachpisinon as Anamiluäkesekach Käz Kesekach miniu; kon tä miniu kinau 
namäsächkänon enechpe, pon miniu papamochnenon.

Ene io näkami Anamihäkesekach kaies ani nawachkikin.

I.

Täpänemenach o kikitwon pächtamokon, käz achpichtänichtakwot ot aiakoamechnesenon; wäwänin pächtamokon.

Nekoneneu Mäz Hawätok; wächzetau kätin kinau menazehau : iachpez änekochkitähäon kinau tapanau.

Kon nasop Mäz Hawätok kinau wawechnanon; kon käkoch miniu nasop kinau wawechtanon.

Käz Kesegot kinau menazechton; Kaiäz Kesekachkin miniu kon käkoch kinau enanochkänon.

Kochne kikiach miniu kinau menatänenawak: kinis asau katäu pemateseon ios achkihe.

Kon weiak kinau nänanon: kon miniu weiak kinau sechkananon.

Pon pachpis maze esetach: maze inänichtamowin miniu zikakiach enäsønach. 
Pon woiak kimotemin : pon miniu weiak waiäsemin.

Kakik kit onamim käkoch äianin : kon tä miniu nekotoch weialk kinau patamo. non.

Pon mochnawenach maze wikichtwon: one nesik anamihäu nepowin.

Pon mochnawenamowin weiak käkoch ächtanachkin, asau katäu kimotemot.

Ene äz Täpänemenach; papamächtawake kakik kinau onanekose: ki nochtamake tä, kakik kinau sächsänänachsi.

Käz achpechtänetakosit Wäwochneme misik Wägiseme misik Waieskesit Hawätok.

Iachpez käz achpechtänetakosit käios iochpe miniu, achpäniu miniu, kakikächkamik. Amen.

\section{II.}

Täpänemeon, wänitchionechse achpäniu achpechtänemena: nanas asau nepäion.

Kakik neu täpänechtach netäh : ki täpänechtahin.

Ene kätin achkano: aiäz matä as pematescon. 
Iochpe tä ki pis nasächkon : pon paketänemena.

Mäz Hawätok Täpänemeon : ki paketänamon nitätchiak.

Ki kes akuasemèmepach : Mäz Hawätok kakik wänamion.

Mawau neu pämateseäk kä achpechtänemächkon Mäz Hawätok: käz achpechtänemächkon tachnäno waichsiäk.

Wenä kakik ki sawänemekonau: mawau tä miniu eneko äz, kakik onamimakat.

Käz achpechtänetakosit Wäwochneme, etc......

\section{III.}

Mäz Hawätok Täpänemeon, zenapamena: osam ne kätemakesim.

Käz manäton ne päzetonon: kon nekotoch pos ani akechzekatäwon.

Iachpez neu ne sechkänechton mächkawänechtamonin kaies pis esekeon: osam ni kes maze pematesim.

Ne kochkoatesemit kä kesekoch pos änapeon : ne kochkoatesemit kä mamazetawak pos waikimakoa, 
Anamachkiach achkano pos ni kes tanächnasim : kat kes suwänemeon.

Mitchineu tä ki mäzemänemim kit änänechtamowene: wächzetau ne sawänecbtakosim.

Mäz Hawätok Täpänemeon : wächzetau kätin net aninichsim.

Kitämakänemena, kinä iachpez nähe sawänechzekäon : sawänemena, Kikis Jesus 0 wichswon io sawänemena.

Mawau neu kesekoch ächtanachkiäk, otchichtchikoanekapawetochkon $\mathrm{Mäz} \mathrm{Ha-}$ wätok: wächzetau asau keketotamaweiek. - Käz achpechtänetakosit Wäwochneme, etc......

\section{IV.}

Iacbpez mamachkatänechtakoseu Mäz Hawätok: äsis sawänemazin kakik näpuachkanit.

Kon käkoch nan ot äsis, sawänemanon : au io piatätoa kakik täpesewene.

Pon mochnawenamowin maze äsechzekätoa o pämatesiniwau: pon iach anächkinon päzeton mihikone. 
Wepoz as nepopimatek asekon: ene äsikimakach päzitatoa.

Kinach tä Mäz Hawätok nesik achpäniminon: waieskewach nesik nanakatawänechtach.

Tapatach Mäz Hawätok o käshiatesin: au isis natawänichtamimakach kitäh, kinau isis ochtänamak.

Käkoch kätau esekeanin, pakichsenemin Mäz Hawätok: kinau wetochkawenemik.

Kinau wasinakichtamihek as kiskanitipächkiseon: tanachsok naiawachkikin kinau isis wasinatisim.

Mäz Hawätok ot änänichtamowin äne kächkenawatänechtach : pon nekotoch ani pakichsenemin.

Pon mochnawenamowin maze pämatesitoa ot onanekosiwiniwau: nemowach nesik onanekosewak.

Kitämakesiwak enänechtakosewak mäze äsetatoa: winua ächpez enänechtakosewak maiächno isikitoa.

Käz achpechtänetakosit Wäwochneme, etc...... 
166

\section{V.}

Täpänemeon, käz kitämakesewene ki ioch keketoton: Täpänemeon, sawänochtawena äsis pakichsenemenon.

Kechtawake ioch pächta: sawänechtach ne mamiachtozekan, ne jakichsenechtamowin.

Kesp̣in nanakatawänichtamawatoa pämatesitoa o maze pematesiniwau: 'Täpänemeon, hawänetowak pos äs mächno inapaminakisit?

Täpänemeon, kit oweawenotan sawänechzekan : as onamion tä miniu ki sawänechzekan ne ioch achpänemim keau.

Nitätchiak achpänemo Mäz Hawätokon ot aiakoamechnesine: nitätchiak achpänemo Täpänechzekänit.

Wepoz os pis wapach nanas as wanitipächkach : nitätchiak au achpäuemo Täpänechzekänit.

Iachpez sawänechzekäu otähe Mäz Hawätok: ki kes pemazehekonau as tanenach kächnap maze hawätok.

Opäsik wächzetau kat ki pakichsene- 
monau Mäz Hawätok : asau kisehamonach ki maze esekenenawon.

Käz achpechtänetakosit Wäwochneme, etc......

\section{Nekamon.}

Mawau neu tä nähechtawon,

Mawau kaies kesechtawon,

Mawau neu känawechtawon,

Mawau neu täpänechtawon.

Kinä wächtenamoweach,

Nekoch äsis manäseach;

Kinä kä känaweheach,

Ki nähe sawänemeach.

Kesik kaies kesechtawon,

Wanitipächkon tatepa, Achkäu tä mawau miniu, Kaies osechton nenach io.

Achpäniu nochtaweach, Kaieketotonanin kä : Nekoch äsis manäseach, As paketenamaweach. 
Tachnäno käkoch matächkin

Kätau nochkehekoiachkin,

Kinach kinuaweiamä, Asau asiänechtamach.

Enech au echpänaseach,

Näniu Nekot Aweion :

Wäwochneon, Wäkiseon

Hawätok tä Mächnoion.Amen。

Neanektochkin aiom nekamon.

Jesus mächnoion,

Käz wäkiseon tä

Käz onät Marion,

Kakik wäne Kekach。

Wieskeseon Marie,

Kesik pachkenamon,

Keketotama kä,

Näwake as kesik.

Ewa, nätom wewon,

Kes päzeton iachpez ${ }_{s}$

Kinach nap nekotoch

Kon päzetoninon。 
Marie käz Nikia, Kekach peneseon,

Pächkonin achpäniu

Ne päzetanenon.

Kit ochtateseu tä, Åsis Kekach wäne, Kesik Mäz Okemau, J esus tä tepenau.

Ki tapanik Jesus, Käshiateseon, Kinach pächtak Wenach, Kakik Mäz Hawätok.

Kinach, Kekachkoion, Pachkinamowatoa Mawau kekachkok neu, Käshiateseon.

As pemateseach

Kakik kesekoch t艹̈,

Näwake as Jestü,

Keketotama kï. 


\section{Käz Näniu Nekot tä,}

Käz tapananonau,

Jesus Okiän kä

- Marie käz tapanau. Amen.

Kaiekachkoit käz Marie o näkamon.

\section{MAGNIFICAT.}

Nitätchiak: käz achpechtänemäu Täpänechzekänit.

Wenach nap nitätchiak onanikınichton: Mäz Hawätok as ioch pematesit.

Kes kanapamäu kä änochkitakot: täpachnenichsinit.

Ene tä sawänechtakosit au äz esekit: mawau neu au enim manazetawitoa.

Iachpez neu ni kes käz totak: mesas käkoch nähechtok tä miniu kaiäz achpechtänctakosit。.

O sawänechzekan au otächnekok kochnatoa: achpäniu asau aıatä pämatesitoa.

Kes wapachtäsewäı äsis tatachkesimakach onä: kes säsiwäpenäu maiamenatesinit otähe.

Kaiäz achpichtänichsinit kes nakichka- 
wänechtamihäu : täpachnenichsinit tä kes käz achpechtänetakosehäu.

Pächkatänit kes achsamäu waieskewach : kaiäz tanamänit tä kes mewenäsehäu, kon käkoch o kes menanon.

Kes mächno otachpenäu Israel änochkitakot: kes mächkawänechton o sawänechzekan.

Kaiesis wawechtamowazin ki mächsomächsinau: Abraham, mawau minıu onitchionächson, ochsechsächson, ot aiatä ochsechsächson mmiu.

Käz achpechtänetakosit Wäwochneme, etc......

Ene iochpe as anamihat Mächkotächko. niäu, nawenau tä miniu ixpänom Jesus Weau. Anamihan ätamä enechpe ïspenachkin Jesus Weau.

Jesus Mäz Hawätok, kinä kaies meseach käz mamachkatänechtakoseu Eucharistiwin, ach్äniu asau mächkawänechtamach kaiesis kotakechtawon nenach io: ki pakichsenemekem asau isis menazechtawach Keau tä ki Mächkom miniu käz Eucha* 
ristiwine ätäk, achpäniu asau mosechtawach nitähinoa kaiesis mächno totaweach as kes akuachsemeach. Amen.

Hawätok Nochne, ne koskänechton, ne käz koskänechton ne päzetonon io; mesem nach tä miniu ki sawänechzekan kesekoch wächpimakach. Kätin ki tapanin, ki katäu tapanin tä kakik miniu. Ki pakitänamon mawau net änänechtamowin, mawau ni kikitwon, mawau tä miniu net äsechzekon. Åsis mininichtamon kä ne katäu esekin. Amen.

Ostiwine sikihenach

Paiachkinamon kesokoch.

$\mathrm{Ne}$ naniwehekomenau,

Sochkitähäskaweamä.

Hawätok käshiateseu

Nekoneneu menazehau,

Tanachkiamake kesik,

Enis asau mininechtach. „Amen。 
KAT KIMÄNAZEHONAU TÄPÄNECHZEKÄГ KÄZ EUCHA. RISTIWINE ÄPIT.

Näniwanäkin kaies ioch osechtok Jesus Käz Ostiwin. Ene kä :

1. Asau pakitänichsit, tachnäno Mäz Anamihar, kaiesis pakitänichsit kinach io as kes akoze anamihäwachtiko.

2. Asau achzamenach Weau misik tä $O$ Mächkom Käz Sawänechtakosine.

3. Achpäniu asau wekemenach anamihäwigamiko Käz Ostiwine.

Kisähächkoa, tipähächkoa tä miniu Jesus asiu anamihäwigamiko; ene tä enoch wä tasit asau ki nasächkawonau, asau ki keketotawonau, asau ki natozikä kwaiachk pämateșin o sawänechzekon miniu.

Saiochkitähäskawatoa wawepoz pis nasächkawäwak Täpänechzekät, kinis miniu anamihäwak. Kaiäz achpichtänichtakositoa iochpe kesekoch äpitoa, ächtasitoa as kes pämatesitoa omos achkihe, neanekotochkin tipähächkoa kes anamihäwak, menazechtawak Jesuson, tapanäwak miniu. 
Kinä miniu wawepoz pis nasächkawin Täpänemenach, mämik Anamihäkesekachkin, neanekotochkin miniu änochkıkesekachkin, Jesus tä kinau käz mächno totak nekoch kesekoch. Iachpez weskiwach asau pichtikäon anamihäwigamiko kaies ani nächkan mitchichsi. Kaies pichtikäon tä anamihäwigamiko, otachpenach akamiha- n wapo, anamihäwachtikonikenon, otchichtchikoanekopa, änekochkitähäon miniu anamihanon.

Menazehin Täpänechzekät Käz Ostiwine äpit, mamiawamin as kes natomik asau isitawat, tapasin, mesin kitäh, natotamawin tapachnenichswon, kwaiachk esekin, misik tä Hawätok o sawänechzekon. Keketotamawin pämatesitoa näpikoa miniu, mämik kinekchekok, kinitchionechsok, mawau miniu paiäz isikitoa, kätau anich pämatesitoa miniu.

Tapatach menazechtoch miniu Jesus Otäh, pakichsenemin tä miniu Hawätok Jesus Otäh asau io sawäneme, asau sawänemazin tä miniu mawau kaikitotamawatoa. 
Aiatchkwot tä keketotamawin käz Marie, käz Joseph, käz Mishäel tä miniu.

Anamihan ätamä weiak asau isitawazin Täpänechzekänit anamihäwigamiko $K \ddot{a} z$ Ostiwine äpenit.

Jesus Täpänemeon äpeon Käz Ostiwine n̉enach io, wäwänin kit änin as kes sawänemeon asau isitonon ios anamihäwigamiko. Sawänemena iachpez änekochkitähäon asau anamihaion.

Ki mamachkatänemin äsis tapaseach as päz esekeach. Achpäniu kisähächkoa, tipähächkoa tä miniu kitim ios anamihäwigamiko, asau nasächkakäon, asau pachkinamakäiach netähenawon, asau wechtamakäon tä miniu äsis päzetaiach, äsis kitämakeseach miniu, ene asau sawänemeach.

Jesus Täpänemeon, änekochkitähäion ki tächtanänemin ios $\mathrm{Käz}$ Ostiwine. Ene omos as äpeon äsis Mäz Hawätokeon misik tä äsis mamazetaweon. Ene ios as atäk kaiäz Keau miniu kaiäz Ki Mächkom; pachkishigon äsinakosit ki kiasim, 
Jesus käshiateseon. Ki kes etan kä: "Pachkishigon, au mikion, ene ne metche"mechsim au ioch pematesitoa mamaze"tawak." Aiatchkwot tä as kes wechponuazin o kächkenohamawekonon ki kes etan: "Otachpenamokon, mezekon tä miniu, Io kä Neau. Otachpenamokon, menäkon tä miniu, kinua mawau, Io kä Ne Mächkom.” Kätin ki pächton eneko äion, Jesus Nochne; sawänemena, opäsik asau papamächtamon.

Ki mänazehin, Jesus Täpänemeon, kit anamihäton miniu. Ki mänazehekäm achpäniu miniu ki käz achpechtänemekok käz achpechtänetakositoa kesekoch äpitoa, ios achkihe tä käz mäse saiochkanamihatoa ki mänazehekok Käz Ostiwine äpeon. Nastä kat ni wichkichton asau isis menazehenon, winua äsis menazehächkoa! Ki mänazehin, Jesus Täpänemeon, ki käz achpechtänemin miniu. As kes akozenon unamihäwachtiko, ki kes kiaton ki Mäz Hawätok, ios tä Anamihau Atuchpone ki kiaton miniu ki mamazeta- 
win; wächzetau tä ki tächtahänimin ios Käz Ostiwine, ki mänazehin, kit anamihäton miniu.

Ki tapanin, Jesus käshiateseon, ki menin tä miniu netäh. Nenä kaies io nepäion anamihäwachtiko asau akuachsemeon, nenä io asion Käz Ostiwine, wawepoz tä miniu kit achsamim tepenau Keau, ächpez tapaseon. Kätin ki tapanin, Jesus käshiateseon, ki käz tupanin, änekochkitähäon ki tapanin, achpäniu tä miniu ki katäu tapanin. Ki menin netäh; sakächnasach netäh; opäsik opäsik asau tapanenon. Amen.

Ene iochpe pos waiechtamä anamihanon misik napan.

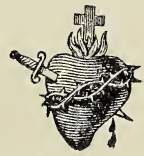


178

\section{KÄZ OTÄH JESUS.}

Kat ki mänazechton Käz Otäh Jesus, kat ki pakichsenemonau miniu Täpänechzekät asau sawänemenach mawau kätau anich pematesinit.
Kakichkotakan.

Käz Otäh Jesus ki käz tapanekonau. Mämik iachpez tapanäú päzetonit kwaiachk aianinichsinit, kätau iachzichtokoa miniu o maze pematesinowau. Käz Otäh Jesus käz kitämakänemäu mawau anino kätau anich pematesinit; naszeau asau aninichsinit, wäwänin miniu paketchekenit, ene tä asau mächno nipäkoa, inänemekok. Ene kätau io tapatamon achpäniu Käz Otäh Jesus, ene kätau io menazechtawon miniu iachpez änekochkitahäion wäwänin. Tachnäno kesekach pos ki pakichsenemäu Jesus, äsis sawänechtach asau sawäneme mawau päzetonit iochpe as kesekach kätau anich pematesinit, asau aninichsinit, wäwänin miniu asau paketchekenit, ene tä asau mächno näpenik, kesekoch miniu asau isianit. Ene kätau iọ ean tachnäno 


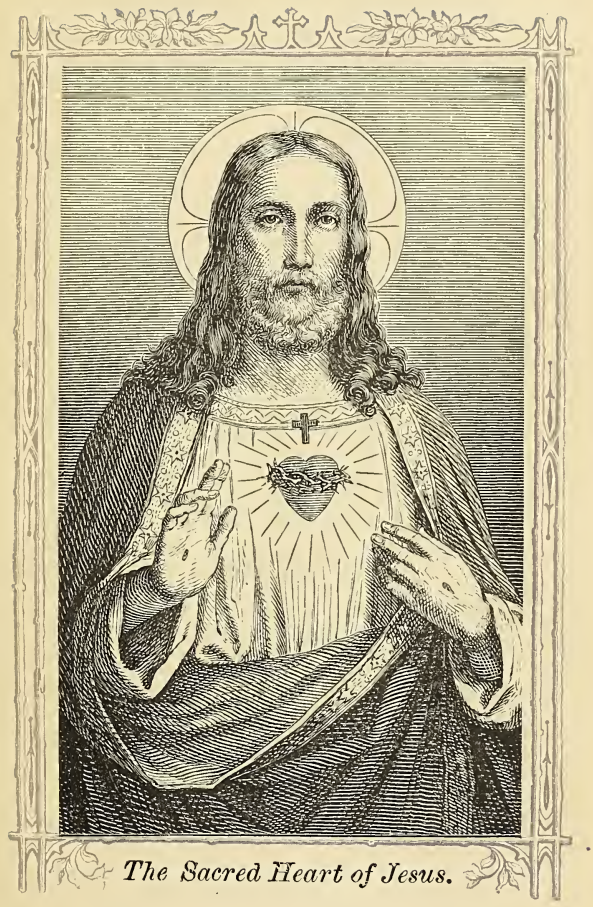



kesekach nekotono: "Nochnenau"...... "Kit anomächkon"..... asau menazechtawon Käz Otäh Jesus, asau sawänimikitoa mawau päzitatoa kätau anich pematesitoa änekochkoa achkảu.

Kätin manäwak iochpe as kesekach kätau anich pematesitoa achkihe. Shuasik metatach tachnäno metatach noachk nepok tachnäno kesekach!

Shuasik metatach tachnäno metatach noachk tepachkonawak!...... Manätoa tä akiko anamachkiach kakik iskotia kätau achpakinatoa. Kätin iachpez manätoa akıko käkiskachkoa käz päzetun; kon aianinichsitoa kätau anich pematesitoa, kakik iskotia au isiwak. Ki pakichsenemekem Käz Otäh Jesus asau sawänimatoa, asau keketotamowatoa miniu päzitatoa tachnäno kesekach kätau nipäkoa. Kespin kwaiachk aianinichsitoa, paketcheketoa miniu äsis wichkichtokoa, au kisehamowawak o päzetonowawon, kes nipäkoa tä kon onau näminowawon kakik iskotia anamachkiach. Ene kätau io pakichsenemot Jesus, ächpez sawäneme asau katäu 
menat anino kätau anich pematesinit waieskewach aninicbswon, ene tä asau mächno näpenik.

Kespin wäwänin keketotamowatoa kätau nipäkoa misik kinach kinau keketotamakok kez pematesewak katäu anich pemateseon, asau mächno nepäion kesekoch miniu asau ision. Kätin manäwak kätau inänikatoa, kälau pichtikanatoa miniu kesekoch, kespin wäwänin anamihätawatoa. Konemau nekot kätau inänihat tachnäno kesckach wäwänin anamihaion, konemau nish, konemau opäsik mäse. Kon ki kächkenanon iochpe, ane enekoch au piatäwihatoa enis kesekoch, echpe tä piatäion Hawätok ot okemawine, kinau kächkenan ene, kakik miniu kinau käz ioch onanikinechton.

Anamihan kätau anich pämatesitoa io.

Jesus käshiateseon, ki käz tapanawak nitätchiakonawak, ene wä pakichsenemenon, Käz Kitäh as wechsakänechtach ki io pakichsenemin, käz Ki Mächkom asau io kishiapawaratoa mawau neu paiäz 
isikitoa mesas änekochkoa achkäu, eneko katau anich pematesitoa iochpe as kesekach.

Käz Kikia miniu, päzeton kat as kes kikehochtatesit, kaies käz wechsakänechtach otähe, ki io pakichsenemin asau sawänematoa käıau nipäkoa.

Jesus Täpänemeon, kaies käz wechsakänechtomon Kitähe as kes iskoanameon anamibäwachtiko, ki pakichsenemin asau sawänematoa kätau iskoanamitoa.

Käz Marie, Wäkiachsemø Hawätok, wächzetau ki mamiachtomin asau k.ketolamawatoa mawau iochpe as kesekach au nepäkoa achkihe.

Nas tä kat keketotamawawak tä miniu kinach, käz Joseph. Amen. 
182

\section{VI. \\ ANAMIHÄU PEME. \\ Kakichkotakan.}

Aiäz nianonik Käz Sawänechtakosinon one kä dnamitıäu Peme. Kat ki nanakatawänechta iochpe äsikimakach ene Kaiäz A chpechtänetakosin.

Kon mawan änamihatoa ot otachpenanowawon ene, one nesik waisakesitoa wätachpinachkoa; kon miniu mawau waisakesitoa ot otachpenanowawon, ene nesik kaiäz wechsakesitoa, nainamesitoa asau nepäkna.

Hawätok o masenaehigone asachzckatäll,asau otachpınachkoa ene waiesakesitoa. Iom kä etan enis: "Wesakesea. weiak ächtaseäk? Naskat au natomäu Mächkotächkoniäwon; wenach tä Mächkolächkoniäu au keketotamowäu waiesakesinit, au anamibäu nomineu miniu, Täpänemenach o wichswone. Ene tä onamuon anamihan ene an io inänit waiesakesi1," (kespin Hawätok enänechtach). (Jacobus v. 1f, 15.) 
Ano kon papik eno waiesakesit onau otachpenamon Anamihäu Peme; koneu nesik käz wesakesit, enechpe au otachpenach. Kon tä weskiwaton osam nauä asau otachpenach; saiä sakanach katäu nepäk. As mäk kä mitchiniu wäwänin kächkenach, au otachjenam. Pon tä weiak kat inänechtam: Kespin otachpenamon Anamihäu Peme, ene kütin asau nepäion, kon nenau inäninon. Pon werak ene kat enänechtom, ene tä asau io sakinimit, wepoz asau otachpenach Anamihäu Peme. Kon weiak o io nepänon, as otachpenach ene kaiäz achpichtänichtakoa Saw ̈̈nechtakosin; pochz naianekotorhkin weiak ioeh inäniu, kespin kä enänechtach Täpänemenach.

Wäki tä maienekosit waiesakesit Anamihäu Peme?

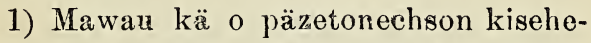
katäwon, mawau miniu enekoch o päzeton] non kaies wa

2) Hawätok o sawänechzekon miniu menekoseu waiesakesit, sinawaneu asau kotakänechtach ächpe» wesakesit. 


\section{4}

3) Sochkitähäskawau miniu waiesakesit, asau sakozehekot maze hawätokon.

4) Naianekotochkin miniu waiesakesit ioch inäniu Anamihäu Peme, kespin Hawätok enänechtach.

Ane tä au esetot weiak asau mächno otachpenach Anamihäu Peme?

Wäwänin kä au paketchekäu, achpäniu miniu Hawätokon au achpänemo.

Wäki tä Mächkotächkoniäu wä nominazin waierkesinit oskechsikoch, ochtawake, otchiase, otone, onächke, osete miniu?

Kit ochkä kiskesikonawon, kichtawakenowon, kitonenawon, kitchiasonowon, mawau kä keowawon as päzetaiach, ene tä Mächkotächkoniäu wä anamihätawazin waiesakesinit, anamihäı nomineu, asau sawänemekot Hawätokon, asan kisehamakonit mawan kaiesis päz esekit as kes maze ok oskechsekon, ochtawakon, mawau miniu weau.

Tane tachnäno weiak pos waichkichtok asau anamihäı nomine? 
Nekotono kä nesik waiesakesit weiak, ene nesik nekotono asau anamihäu nomine. Inänit tä, misik tä wesakesit, ene tä misik asau wichkichtok asau anamibäu nomine.

Kakichkotakan Waiesakesitoa 10.

Wäwänin otachpenach ki wechsakesin, Kochne aiachpez käshiatesit kesekoch äpit kit anänemik newemach asau wesakeseon. Kespin sasipänerhtamon, kinau menik kakik onanekosin kesekoch. Ki wechsakesin wepoz au ani takimakat, sawänechtakosin tä kesekoch ätek kon nekotoch au ani atäwon.

Mächkawänemin kesekoch iochpe ächtanesitoa äsis sawänechtakositoa! Nekot nitchior. kes£koch äpit opäsik sawänechtakoseu, mawan tä pämatesitoa achkihe äsis sawänechtakositoa. Kinau menekem tä niniu ene käz sawäkechtakosin, kespin tro wäwänin kotakechtawon nemewach omos achkihe.

Mächkawänechtach kakik kotakechton anamachkiach! Konopoz ki kes wichkoazichtoasimepach, kakık iskotäu asau ta- 
nächnasion. Ki sawänemik tä Täpänech. zekät; kon ki kes achuakenonon kakik iskotia; kit änänemik tä mänawaz nesik asau wesakeseon, kes nepäion tä ki katäu otachpenik kesekoch. Kätin iachpez kishialeseu! Wänitchionächse kit ächpenanik, wänitchionächse kit äsis tapanik.

Mächkawänechtach miniu äsikimakach kishichnasoane. Kätin iachpez wesakä nechtamuk enis aitoa. As kes päzitatoa ano mänawaz, ene wä kotakänechtachkoa. Konopoz mäse ki kes kikäskamuk päzetonechson; kes nekatamon achkäu as mäk kikäskamon päzetonechson, kinis kone-mau poz ki wechsakänechton. Wepoz tä kesekoch asau pichtikäon kes anich pemateseon achkihe, ene wä sächsewaho iochpe Täpänechzekät, ki päzetonon ke io sächsewahok. Kätin ki sawänemik! Pon tä inänechtach: Ne nächkenemik Hawätok; pon ene inänechtach; enänechtach tä: $\mathrm{Ne}$ tapanik, wänitchionächse ne totak Nochne kesekoch äpit; wepoz ne katäu otachpenik kesekoch ene wä säsewahit mänawaz iochpe. 


\section{7}

Mächkawänechtach Jesus o kotakechton. Zenapamin anamihäwachtik! Näniu tepachikon kes käz wechsakänechtam Täpänechzekät, mawau weio kes mächkoweu, nianon tachnäno kes enakanamau, anamihäwachtiko kes tachpänäu. Zenapamin anamihäwachtik! Ki Hawätok täpane kes käz anemeseu, kon pochz mï̈nawaz ki katäu anemesenon? Wenach kon nekotoch o kes päzetonon, kinach tä konopoz wawepoz ki kes päz enänechtom, konopoz wawepoz ki kes jäzetom, konopoz wawepoz ki kes päz esetom. Ene kätau io otachpenamon ki wechsakesin mawau anemesin eneko maiene Täpänechzekät.

Waiesakeseonin kene, mächkawänechtach kaiesis kotakechtok Jesus wene, as kes pachsäkipene minichsakächsin. Kes nepako tä mächkawänemin Täpäneme, kaiesis nepakit as mäk akoze anamihäwachtiko, kes keketo: "Ne näpakim." Kon tä nepeu kes menanon, sewapo kes menabäu. Kespin tä naianekotochkin tä- 
$18 x$

wächkion, täwisitäon miniu, mächkawänechtach kaiesis wesakänechtach Täpänechzekät, anamihäwachtiko as kes sachsakachkoho onächke osete miniu. Kespin mächkawänechtamon achpäniu Jesus o kotakechton, opäsik wäwänin kinau sasi* pänechton waiesakeseonin. Achpäniu pa. ketenamowin Kochne käshiatesit kese. koch mawau eneko kotakechtawon, asau katäu kotakechtawon, Jesus kaiesis kotakechtok. Kätin kinau käz mächno esech. tom, Hawätok tä kinau käz mächno ech. pänonik kesekoch. Ene tä au esetaion, ächpez wesakeseon; kächkenawapomin Jesus, kaiesis kotakechtok, kotakechtach miniu kinach; kaiesis sasipänechtach, sasi pänechtach miniu kinach ap.

Iachpez änekochkitähäon onamichtach Hawätok o kikitoan. Kon nekotoch nasop o kikitinon, ene au io onamichtawat eneko kaikitit. Mämik onamichtach eneko waiechtamoch iochpe, ene kä :

1.) Tanäseu Mäz Hawätok nekot; iachpez käz mächno esekeu, iachpez käz kishiatescu miniu. 
2.) Näniu Nekot Aweu Mäz Hawätok, Wäwochneme, Wäkiseme, Waieskesit Hawätok. Mäz Hawätok aweu Wäwochneme, Mäz Hawätok aweu Wäkiseme, Mäz Hawätok aweu Waieskesit Hawätok. Kon tä o näninon Mäz Hawätokok, nekoneneu nesik, Näniu Nekot Aweu Mäz Hawätok.

3.) Kwaiachk tepachkonikäu Hawätok, maiächno esekenit au pichtikanäu kesekoch, kakik enis asau sawänechtakosinit; mäze esekenit tä au achpakenäu anamachkiach, kakik iskotia asau winosinit.

4.) Kwaiachk Mäz Hawätok aweu Jesus Krist, Mäz Hawätok Okison. Kakik Mäz Hawätok aweu; asau inänihazin kitätchiakonawon, ene kätau io nıamazitawihäsit, ene kaies io kotakechtok, ene kaies io tachpänät anamihäwachtiko.

5.) Kakik au pematesewak kitätchiakonawak, kon nekotoch onau nepänowawon. Mächno esekewak kakik au mächno tanäsewak kesekoch; mäze isikitoa tä kakik anamachkiach au wechsakänechtamuk.

6.) Ene nesik Hawätok o sawänechzekon wä wichkichtawon käkoch asau 
mächno esetaion, kätan io wichkichtoaseon kakik pematesin kesekoch. Ene kätau io pakichsenemot achpäniu Hawätok asau sawäneme, asau mene o sawänechzekon, asau wasinakichtok, asau moskawänichtamihe miniu, wäwänin asau anochkitawat Täpänechzekät, ene tä kakik asau sawänechtakoseon kesekoch.

7.) Nekoneneu nesik Kwaiachkichnin Anamihan, ene kä, Katolik Anamihan; Jesus tä kes osechtau ene Anamiban; ene kätau io onamichtamon anamihäu kakichkotakan eneko kakichkeme Mächkotächkoniäu.

8.) Täpänechzekät Jesus kes esechtau nohekon käz Sawänechtakosinon, ene kä : Sikahapuanitwon, Sochkitähäskatwon, $\mathrm{Pa}$ ketchekon, Käz Ostiwin, Anamihä u Peme, Mächkotächkoniäwiwin, Anamihäu Wikichtwon.

9.) Käz Ostiwine, kwaiachk tanäseu Jesus äsis Mäz Hawätok awit, äsis mamazetawit miniu pachkishigon somenapo miniu ot äsinakosine. 
10.) Mäz Hawätokon kes menik Mächkotächkoniäu ene tatachkesin, asau wichkichtok, asau kisihamawikit päzetonon, kespin päzetot kwaiachk aninichsit, paketcheket miniu wäwänin.

11.) Jesus kes osechtau Anamihäu Peme, waiesakesit asau io inänit; kespin tä Hawätok inänemekot asau nepäk waiesakesit, Anamihäu Peme au io wetochkawau, asau mächno nepäk, ene tä kesekoch asau isiat.

Ene kätau mächkawänechtamon, kätau onamichtamon miniu.

Pos kit achpänimin miniu Hawätok. As kes päz esekeon; ene kätin; Jesus tä ki kes nipotak, Hawätok asau ponikitätoch eneko kaies maze esetaion; kes nipoa Jesus asau inänion kitätchiakon; kes nipoa, kakik iskotia asau akuachseme. Ene kätau io achpänimion Hawätok, iachpez sochkon achpäniminon. Jesus o kotakechton io kinau ponikitätok, kes nepäion achkihe, kinau otachpenik kesekoch.

Tapasin Hawätok; kätin käz achpechtänetakoseu! Iachpez mächno esekeu, 


\section{2}

iachpez kishiateseu miniu. Wänitchionechson ki totak, kit äsis tapanik miniu. Jesus tä kes paketänechtom o pematesin anamihäwachtiko misik tä kes tachpanäu, kaies iachpez sawäneme, as kes iachpez tapane. Tapasin Hawätok, achpäniu tapasin, iachpez änekochkitähäon tapasin.

Kinau tapanik miniu kez pämatesewak, Hawätok tä ene au io tapanatoa. Ponikitätawin tä kaies maze tasimächkoa, kaies maze echpänonachkoa miniu. Pon weiak sechkasin, pochz saiechkane ponıktätawin, Jesus kaiesis ponikitätawazin kaies sechkanekot. Kespin tä käkoch kes kimotemot weiak, iach mesin, tepahamowin miniu ki masenachegon äsis wichkichtawon.

Aninichsinon kitähe; ki päzetonon io koskänechtach monon miniu. Kespin kon aninichsion, kon nekotoch kinau kisehamakänon Täpänechzekät; kespin kon kisehamakäon asau konomächtchiu anich pemateseon achkihe, kakik kinau käz wechsakänechton anamachkiach! Kätin 


\section{3}

kotamikwot ene wechsakänechtamoan anamachkiach! Ene kätau ioch aninichsion, iachpez änekochkitähäion aninichsinon. Wawepoz natotamawin Hawätok waieskewach aninichswon. Koskänechtach kitähe as kes päzetaion, as kes nächkehat Hawätok; iachpez mächno esekeu, iachpez tapanckoseu, kishiateseu miniu, wenach tä eno kaies rächkehat, kaies maze echpänanat. Mächkawänemin Täpänechzekät kaies tapane, kaies sawäneme miniu as kes nepäk anamihäwachtiko kinach io. Wawepoz wechtach wäwänin ene anamihan, aninichswon as wechtek.

Kotakiheswone otachpenach ki wechsakesin mawau miniu eneko kotakechtawon. Pon nächkosenon, pon miniu nächki keketotawin weiak. Mamiawamin achpäniu känawehe, achkosik, ächsame, mänahe. Wawepoz anamihanon, mämik -anich pemateseon. Onamichtawin Hawätok, achpäniminon, tapasin, aninichsinon. Wechtach wawepoz anino niu anami- 


\section{4}

hanon, ene kä: Onamichtamowin, Pakichsänichtamowin, Tapanitwon, Aninichswon. Paketenamowin Hawätok keau, kitätchiakon, ki pematesin miniu. Zenapamin wawepoz anamihäwachtik, otchimin miniu, mächkawänemin tä Jesus kaiesis käz anemesit kinach io. Iachpez wawepoz akemin anamihämiguk asau menazehat kaiekachkoit käz Marie; pakichsenemin tä, Jesus, o kotakechton, Jesus käz O Mächkom io pakichsenemin asau keketotamo achpäniu, mämik echpe katäu nepäion. Mamiachtomin miniu käz Joseph asau kitämakäneme, asau wichkichtoa miniu waieskewach nepäm.

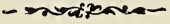

Anamihan wawepoz ätamä ächpez wechsakesit.

Hawätok Nochne, sawänemena asau mächno otachpenamon ne wechsakesin. Kon nenach änänechtamon pos esekenon; kinach kä, Nochne, kinach änänechtamon pos esekin. 
Nas tä kat ne sasipänechton wäwänin ächpez wechsakeseon. Net anemesim kätin, ni wichkoazichtoasim tä opäsik asau anemeseon.

Täpänemeon, ki pakitänamon mawau eneko kotakechtawon. Kaiesis kotakechtawon nenach io, ene kätau kotakechtawon kinach io.

Ki käz tapanin, kakik iskotia miniu ki kes akuachsemim; nenä kaies io sekenamon Ki Mächkom, ene wä tapanenon; sawänemena opäsik asau tapanenon.

Wäwänin kit änin, Täpänechzekäon, kaiesis tapaseon, kaiesis mächno totaweon nanas iochpe. Ne mamiachtomawak mawau käz achpechtänetakositoa kesekoch äpitoa asau mamiawamächkoa, kaiesis mächno totaweon. Mämik wäwänin as kes sawänemeon asau anamihaion, asau sikahapuanekäion miniu. Wäwänin as kes achsameon wawepoz Keau Käz Ostiwine, ki käsehamawim tä ne päzetonon. Nas tä wepoz kat kinion kesekoch, kakik asau pos mamiawaminon, kaiesis sawänemeon nanas iochpe. 
196

Hawätok Nochne, mawau neu kaiesis kächkenohamaweon äsis anamihaion, kinä kaies ean ene mawau wä onamichtamon.

Hawätok Täpänemeon, wächzetau kätin net achpänimim Keau, Jesus o kotakechton io asau kisehamaweon ne päzetonon, asau wetochkaweon miniu wäwänin asau sasipänechtamon nekoch au äz wesakeseon, kes nepäion tä, kakik asau onanekoseon kesekoch.

Hawätok Täpänemeon, wächzetau kätin ki käz achpechtänetakosim, wächzetau miniu ki tapanekem; ene wä tapanenon opäsik mawau tä äsikimakach. Ne tapanawak miniu mawau mamazetawak, kinä wä tapanakoa; ächpez tapanision, ene ächpez tapanakoa.

Sawänemena, Hawätok Nochne, ne päz esekim kätin. Kanawehena, sawänochtawena, Jesus, Hawätok Wägiseme, sawänemena, ki kotakechton io ponikitätawena.

Käz Marie, achpäniu ki keketotamawawak paiäz isikitoa naiatenesemächkoa ; nenach nap tä, ano as päzetaion, ki pis 
natenesemin as koskänechtamon as kes päzetaion. Keketotamawena asau kisehamawit Kikis Jesus. Mawan miniu kesekoch äpitoa, keketotamawekan mämik echpe nepäion.

$\mathrm{Ne}$ paketänemau maze hawätok, ne pakitänechton mawau ot änanochton, mawau miniu o maminatesin ne pakitänechton. Kinuawin enoch maze hawätok kon käkoch asau echpänasit katäu anich pemateseon.

Kon net achpechtänetakosenon os pos pichtikäon kesekoch, osam ne maze esekin. Net achpänimin tä Kikis Jesus. Nenä kaies io tachpänät anamihäwachtiko, ene wä achpänimion Keau, Jesus o kotakechton asau io kisehamaweon ne maze esetonon.

As kes päzitatoa nätom mamazetawak, ene kries io inänemeach asau nepäiach. Kinach änänechtamon, Hawätok Nochne, naskat au esekemakat. Ne mäniriechtor asau nepäion asau papamächtonon; ne mäninechton asau nepäion asau kisehama- 


\section{8}

weon eneko kaies nächkehenon; ne mäninechton asau nepäion kakik asau nämon asau tapanenon miniu kesekoch.

Nas tä wepoz kat ne nämin kesekoch, Nochne käshiateseon! Nas tä kat ne näwawak käz achpechtänetakositoa kit okemawine aitoa. Kätin mininechtakwot kesekoch. Kon nekotoch pos pachkatänon, kon nekotoch pos wesakesinon: kon käkoch sänakach pos takon kesekoch, asit tä kakik onanekosin au takoa. Hawätok Nochne, miakonena achkihe, kesekoch tä pichtikasena! Kätin mäse mäte takoa ios achkihe, enis tä kit okemawine kon käkoch mäte takon. Sawänemena, zenapamena, kit okemawine tä otachpenena.

Ki pächton, net achpänimim Keau, ki tapanin, kinä wä sechkatamon ne maze esekin, ächpez wieskeseon, ächpez tä miniu tapanckäon. Sawänemena, kisehamawena, Jesus o kotakechton io kisehamawena.

Käz Marie, Wäkiachsemenon, ki kes näwau Kikis Jesus as tachpänät anami- 
häwachtiko; keketotamawena echpe katäu nepäion.

Käz Mishän, kaiäz Asäniwion anamihätawena.

Ne mächno asänim, kil anonik Hawätok asau kanaweheon, kanawehena, keketotamawena miniu katäu anich pemateseon.

Kinuawin saiechkasit, kon käkoch asau echpänasit. Kakik kesekoch apeiek, anamihätawekon, asau nianakok, asau wez tanachkiamenakok kakik kesekoch. Amen.

Jesus, Marie, Joseph,

Ki pakitänamon

Neau, netäh, netätchiak,

Ne pematesin miniu.

Jesus, Marie, Joseph,

Mächno kanawehekon

Katäu iskoanamion.

Jesus, Marie, Joseph,

Ishiasekon, asau

Wanachkion, as kes

Nepäion achkihe. Amen. 


\section{Kakichkotakan}

asau kächkenachkoa au isichziketox akiko

känawehatoa waieskesinit.

$\mathrm{Ki}$ käz mächno esetam wäwänin as kanawehat kez pemateseu waiesakesit. "Eneko kaies echpänanekoa nematak opäsik päpiatesitoa, nenach neu ki kes esetam," ene $\mathrm{au}$ eta Täpänechzekät Tepachkonikä kesekach. Waiesakesinit weiak, naskat as nasächkawazin, mächno keketotawin sochkitähäskawin miniu.

Ene tä au enat waiesakesit: Kit änänemik Mäz Hawätok asau wechsakeseon; wäwänin otachpenach ki wechsakesin, sasipänechtach miniu. J esus käz wechsakänechtamopach, as kes pis pemazehenach kitätchiakonawon. Kächkenawapamin Jesus; kaiesis nawaneu esekit, nawaneu esekinon. Pachkichsenemin Hawätok, misik asau inänion; pon tä osam monawena kinis asau pemateseon ios achkihe. Enänechtach tä: kon nenäch änänechtonon pos esekinon, äsis mininechtach kä Täpänechzekät, naskat au esekemakot. Anamiha- 
non wawepoz, mächkawänemin Hawätok, paketenamowin tä mawau eneko kotakechtawon.

Neanekotochkin wapachtach anamihäu masenachigon, akechtach tä anamihanon waiesakesitoa kaies io asachzekatäk. Sinawaneu akechtach, pon tä osam kinis anamihanon, pon tä miniu osam mäse akechtach anamihanon, au wesakeseu kä waiesakesit. Neanekolochkin nekaminon tä miniu anamihäu nekamonon. Atuchpone kächze nepakone ato anamihawapo, waiesakesit asau ok wawepoz asau anamihäwachtikonikät. Anamihäwachtik tä asin atuchpone, asau zenapamazin asau otchimazin tä miniu, ene tä asau mächkawänemazin, Jesuson kaiesis kotakechtonik.

Kaiäz wesakesit tä weiak, awech natomin Mächkotächkoniäu, asau paketcheket waiesakesit, asau otachpenach Jesus Weau, asau anamihäu nomine tä miniu, ene tä nätom as keoseach asau is nepäk. Ponr, pon nekotoch paketenin weiak asau ne- 


\section{päk, konomächtchiu asau niakot Mächko.} tächkoniäwon. Konopoz käz päzeton zekekäskom waiesakesit, kespin tä anich pematesit konomächtchiu asau aninichsit, kakik iskotia au achpakenau.

Nohekon ene peponakesit nitchion wesakesit, awech nasin Mächkotächkoniäu, asau paketcheket nitchion, asau anamihäu nomine miniu. Poz käz wanau it Mächkotächkoniäu, poz käz käsik, konopoz kimewan, naskat awech natomin Mächkotächkoniäu, Hawätok tä kinau mächno totak nekotäs kesekoch.

$\mathrm{Au}$ paketcheket tä waiesakesit, mawau neu asan kisehamo eneko kaies maze esetot, konomächtchiu asau nasächkawazin anino au tepachkonikät.

Au otachpenom tä miniu Jesus Weau; "Viaticum" ene tä as wechtek, enechpe Käz Ostiwin, ene tä, "Käz Niman." Iskoaz tä au otachpenamon waiesakesit Anaınihäu Peme, misik tä asau mächnoit; enänemekot tä Täpänechzekäıit asau anich pematesit, au wetochkak Anamihäu 
Peme asau mächno nepäk, ene tä kesekoch asau pichtiket.

Konomächtchiu asau pis ishiat Mächkotächkoniäu, päz ozechtok asau apet as wekit waiesakesit, Täpänechzekät $k \ddot{a}$ au pichtikäu. Ene au io zekatachamon asau apet, kisechnito tä miniu. Au kisesitä miniu waiesakesit, mämik tä pos kisechninächso, pos kisechnisitäu tä miniu.

Atuchpone ato kächze nepakone, ene 1ä wachkez atuchpone asau atawon wapiskikon, anamihawapo miniu, mänawaz miniu tachkikamiu ämiskoachsa miniu, nekoz nekot wasachkoneganapä, nekot tä miniu amo peme wasachkonegon, mänawaz miniu papakeweian. Anamihäwachtik miniu asin waiesakesit asau zenapamazin. Nekot miniu ato kishinachkon onagon.

Pichtiket tä Mächkotächkoniäu, papik otchichtchikoanetawin, asau menazehat Täpänechzekät Käz Ostiwine kaiasit. Asau paketcheket tä waiesakesit, sakisinon tä nemewach, kes ponetot tä, naskat pichtikenon, kinach mawau miniu ächta- 
204

seäk. Iachpez änekochkitähäon anamihanon; keketotamawin waiesakesit asau misik mächnoit; enänemekot tä Hawätok asau nepäk, keketotamawin waieskewach nepän asau menekot Täpänechzekänit.

Anamihan ätamä konomächtchiu asau otach-

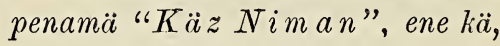

\section{JESUS WEAU.}

Jesus Täpänemeon, ki katäu pichtikäon netähe. Konemau tä ene aiatchkwot, asau otachpenamon Keau. Konopoz wepoz kinau natomin, as tepachkoneon. Ene wä ki pakichsenemin asau sawänemeon asau mächno otachpenenon. Mesenach Käz Niman, asau io pematesit nitätchiak. Ano kon ki nianonenon; Jesus, ki tächtanänemin tä Käz Ostiwine. "Pachkishigon ot äsinakosine kikiasim, Mäz Hawetokeon! Iachpez tapaseon, ene wä kiaseon, kon asau säkiseon, katäu otachpenenon. Kätin ki pächton, eneko äion. Nas tä kat ki mesim opäsik mäse kwaiachk onamichtamoan. 
Jesus Täpänemeon, ki käz kitämakänemawak päzitatoa aianinichsitoa, ene wä achpänemeon Keau, asau katäu kisehamaweon miniu, ne päzetanon.

Sawänemena, kisehamawena, ki wichswon io kisehamawena. Ki kes paketänechton ki pämatesin, ki kes sekenon miniu mawau ki mächkom nenach io, iochpe tä ki katäu pichtikom netähe, ki katäu achsamim Keau ächpez tapaseon. Ene wä achpänimion Keau, Jesus käshiateseon, asau ponikitätaweon mawau eneko kaies maze esetom, kesekoch miniu asau otachpenamon kes nipäon achkihe.

Ki tapanin, Jesus käshiateseon, osam mänawaz tä ki tapanin. Ene wä päkichsenemin asau wetochkaweon opäsik opäsik asau tapanenon. Nas tä as wichkichton achpäniu asau isis tapanenon, äsis tapanachkoa käz achpechtänetakositoa kesekoch äpitoa. Sakächnachsa netäh, asau käz tapanenon Jesus Nochne. Nas tä wepoz nikatamon achkäu, kakik asau tapanenon kesekoch. 
Jesus Täpänemeon, kon net achpechtänetakosinon asau pichtikäon netähe, osam ne päz esekin, wáwepoz ki kes nächkehin, opäsik ni kes achpechtänechton maze onanekosinon, kinä achpechtänemenon. Kätin kon net achpechtänechtakosinon asau pemateseon wachkitachkamik, ne wichkoazichtoasim kakik iskotia asau tanäseon. Sawänemena, zenapamena tä, Jesus nähe sawänechzekäon, pon totawena, kaiesis wichkoazichtoaseon as kes päzetaion. Sawänemena, kisehamawena, ki kotakechton, ki näpin io kisehamawena. Ne käz koskänechton as kes nächkehenon. Nas tä nepäion konomächtchiu as kes nächkehenon! Iochpe tä ki tapanin, ächpez änekochkitähäon ki tapanin, ene wä aninichsion, ene wä koskänechtamon as kes päzetaion. Iachpez tapanenon, ene wä koskänechtamon as kes nächkehenon. Sawänemena, ponikitätawena kaiesis maze esetaion nanas iochpe.

Jesus kinau pichtikäon iochpe netähe. Kätin ne käz mächkawänechton asau 
otachpenenon. Pianon, Jesus pianon, pis tepänechtach netäh, penechtoch netäh, sakächnachsa netäh, ki natawänimikäm, pichtikawena. Nas tä wepoz wapamenon kesekoch! Amen.

Anamihan ätamä kes ani otachpenamäkin

\section{KÄZ NIMAN.}

Jesus apeon netäh, ene asit, kätin ne sawänechtakosim. Pon kaiänit inänechtach, netäh, ene nesik Jesus pos mächkawänemit iochpe. Jesus käshiateseon, wäwänin as kes pichtikaweon netäh. Nas tä wichkichtoeon asau täp mamiawamenon, kaiesis mächno totaweon iochpe! Osam mänawaz ne mamiawamon, ene wä natomenon Wäkiachsemot käz Marie, mawau miniu kesekoch äpitoa, ene wä naiachtomakoa, asau mamiawamakoa kaiesis mächno totaweon as kes pichtikaweon iochpe.

Kätin ne käz sawänechtakosim ki käskawau. Jesus Nochne. Pachkishigon ot äsinakosine ni kes otachpenom Keau, kitä- 
tchiak, kina Mäz Hawätok miniu. Wäwänin, Jesus, iachpez wäwänin!

Ki mänazehin, kit achpechtänetakosin miniu, Jesus Mäz Hawätokeon, ki menazechtakäm miniu mawau achpichtisitoa asäniwak miniu kesekoch.

Ki tapanın Jesus Täpänemeon, ächpez änekochkitäbäion ki tapanin, sawänemena opäsik asau tapaseon. Ki menin netäh, ki tapanekem netäh, osam mänawaz tä ki tapanin, ene wä pakichsenemin wepoz asau otachpenamon kesekoch, kakik asau käz tapanenon kit okemawine. Iachpez tapanenon tä, ene wä koskänechtamon as kes päzetaion. Ki mamiachtomin tä, Jesus nähe sawänechzekäion, asau kisehamaweon ne päzetonon. Säsewahena omos achkihe, pon tä achpakesenon kakik iskotia, kaies wichkoazichtoasion as kes päzetaion. Mächkawänechtach kaiesis wechsakänechtamon anamihäwachtiko asau inänihat netätchiak. Ki kıtakechton, käz ki mächkom io kisehamawena. Net änänechton kon nekotoch katäu päzetaion. 
Kotakihiswone ne katäu otachpenom wechsakesin, anemesin, nepän. Kinach miniu, Nikia, käz Marie, kakik kaiekachkoit, keketotamaweach; Nikia kit äsis tapanin, ki pakichsenemin miniu ki nitchionächsä asau äsis tapaseon. Pakichsemin Kikis Jesus asau sawänemit, asau kisehamawit mawau ne maze esekinon, kes nepäion tä achkihe asau otachpenit kesekoch. Amen.

Anamihan ätamä konomächtchiu asau otach-

\section{penam $\ddot{a}$ \\ ANAMIHÄU PEME.}

Jesus Täpänemeon, ki kes etan: "Wesakeseu weiak ächtaseäk? Naskat au natomäu Mächkotächkoniäwon; wenach tä Mächkotächkoniäu au keketotamowäu waiesakesinit, au anamihäu nomineu, Täpänemeach o wichswone. Ene tä onamuon anamihan ene au io inänit waiesakesit, kespin tä maze esetot, au kisehamawäu Täpänechzekät." Kätin Kikis Jesus kes osechtau Anamihäu Peme asau io wetochkawatoa änamihatoa waiesakesitoa, asau ioch inänihatoa miniu. 
Ene wä pakichseneminon, Täpänemeon, asau sawänemeon wäwänin asau otachpenamon ene Käz Sawänechtakosin.

Inänichtach neau, kesppin mächno eion au io wetochkaweon, mitchiniu wäwänin asau anochkitonon. Enänemeon tä asau nepäion, sawänemena asau mächno nepäion. Kisehamawena mawau ne päzetonon; kätin net aninichsim as kes nächkehenon; ächpez tapanenon, ene wä koskänechtamon as kes päzetaion, kon nekotoch kinau nächkehenon. Sawänemena achpäniu asau sasipänechtamon au ächpez wechsakeseon. Moskawänichtamihena miniu achpäniu asau mekanok asau nochpehak maze hawätok ; sochkitähäskawena kakik asau paketamowak mawau o maze hawätok enänechtonon. Ene wä io otachpenamon Ananamihäu Peme iochpe.

Wetochkawena asau mächno otachpenamon ene Käz Sawänechtakosin. Amen. Anamihan ätamä kes ani otachpenamächkin ANAMIHÄU PEME.

Wäwänin kit änänemin, Jesus Täpänemeon, as kes ki sawänemeon, asau ana- 
mihäu nominit ki Mächkotächkoniäu. Net änänechton tä iochpe: nas tä Anamihäu Peme io inänihion! Enänechtamon tä kinä, Nochne, nas tä esekemakot! Ki paketänamon neau netätchiak miniu! Kon nenach änänechtamon pos esekemakonon, kinach änänechtamon naskat au esekemakot. Sawänemena sinawaneu asau kotakänechtamon, kinach, Jesus, kaiesis kotakechtawon as kes akozenon anamihäwachtiko; ki katäu kächkenohawapamin, sawänemena!

Ki pächton, net achpänemim Keau, ki tapanin, ne sechkaton ne maze pematesin. Ne paketänemau maze hawätok, mawau miniu ot änochkiwinon ne paketänechton. Sawänemena kon nekotoch asau otachpenamon maze änänechtonon kinach tä asau ächpez tapanenon.

Nikia käz Marie, ki nitchionächsä kanawehena, kon asau pächtawazin maze hawätok. Mämik tä anamihätawenach katäu anich pemateseon Mäz Hawätok o sawänechtakosin as tase nepäion, ene tä kakik 
asau onanekosion kesekoch. Käz Joseph, kinach kaies pemekehat Jesus misik tä Marie ios achkihe, keketotamawena katäu anich pemateseon. Net asänim, kanawehena, asau matchiat maze hawätok. Mawau kesekoch äpiäk, wetochkawakichtoa asau mächno nepäion, ene tä kakik asau witchianakok käz onät. Amen.

Kakichkotakon katäu anich pematesitoa io.

Echpe katäu anich pematesit waiesakesit, naskat au anamihat wäwepoz eneko äsis wichkichtok.

Kon tä osam kinis pos anamihanon otone, otähe tä pos anamihat. Poz onamichtamo, pos ächpänirno Mäz Hawätok, pos tapanäu uniniu, pos aninichso miniu änekochkitähäion. Pos neanekotochkin zenopomäu anamihäwachtiko, pos otchimäu miniu, konau tä pos mächkawänechtomäu Jesuson, kaiesis kotakechtonik. Pos pakichsenemäu tä Mäz Hawätok Jesus o kotakechton asau io kisehamakit mawau päzetonon. Kätin Jesus o kotakechton au io kisehamawau, kespın kwaiachk aninichsit, 
konau tä apänimot, tapanazin miniu Mäz Hawätokon.

Kespin tä maze hawätok menekot maze änänechtonon, naskat anamihat papik neu wechsakesit, au pakichsenemäu tä Mäz Hawätok, asau moskawänechtamehekot, wepoz asau nochkehazin mawau maze enänechtanon.

Misik tä pos anamihäu waiesakesit Jesus Okiän, Käz Marie, asau keketotamakot, asau wetochkakat miniu.

Waiesakesit pos mamiachtäu miniu käz Joseph, kaies sochkanemekot Jesuson, mawau miniu käz asäniwak pos natomäwäk, mämik tä käz Mishän kaies okemawit asäniwon. Käz arhpichtisitoa kesekorh äjitoa pos anamihäwak waiesakesit asau mächno nepäk. Wawepoz naskat anamihat wechsakesit:

"Jesus, Marie, Joseph, ne päkitänamon neau, netähe, nitätchiak, ne pämatesin." Ne pakitänamon, Hawätok Nochne, ne kotakechton kaiesis kotakechtok Jesus anamihäwachtiko.

Mawau enotäweseu tä enis ait waiesa- 


\section{4}

kesit pos anamihawak neanekotochkin. Kon tä onau nekaminowanon echpe katäu nepäk wechsakesit.

Mawau tä pos käz anamihäwak, Hawätok asau sawänemazin waiesakesit, asau kisehamawakit o päzetonon, asau menazin tä mächno nepän.

Kon tä osam kinis pos anamihau; wawepez pos säsopinamawau wechsakesit anamihäwapo, maze hawätok asau matchiat. Wesakesit weke äpitoa neanekotochkin pos wapachtomuk anamihäwachtikon, asau zenapamazin otchimazin miniu.

Katäu anich pematesit tä wechsakesit, pos menäu nokot amo peme wasachkonenegon asau kanawehazin nanas as kes nepäk, konau tä au anamihau äsis wichkichtok ächpez änekochkitähät.

Ene wasachkonenegon pos sikahapuane katäu.

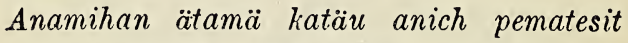
waiesakesit. (Sinawaneu wechtach.

Hawätok Nochne, ki pächton, net achpänemim Keau, ki tapanin. 
Iachpez änekochkitähäon ki tapanin, sawänemena opäsik os pos tapanenon.

Iachpez tapanenon, ene wä koskänechtamon as kes nächkehenon.

Naskat nepäion konomächtchiu asau misik nächkehenon.

Sawänemena, kisehamawena, Jesus o kotakechton io kisehamawena.

Jesus nähe sawänechzekäion, ki menin netäh, netätchiak miniu.

Ki kes tachpänä anamihäwachtiko, asau akuachsemeon. Ene wä achpänimion - Keau, asau katäu ponikitätaweon, eneko kaies maze totaweon.

$\mathrm{Ne}$ käz aninichsim kon as kes tapanonon, kon as kes anochkitonon.

Iachpez tä tapanenon iochpe, ene wä koskänechtomon, ene wä käz aninichsion netähe as kes päzetaion.

Kisehamawena, Jesus, kisehamawena ne päzetonon.

Nikia, käz Marie, anamihätawena;, natomin Kikis Jesus asau sawänemit, kischamawit miniu. 
Käz Joseph ki sochkänemekem Jesus, keketotamawena.

Ne mächno asänim, wetochkawena, asau matchıt maze hawätok.

Mawau kesekoch äpiek, anamihätawena.

Käz Otäh Jesus iachpez sawänemä. käon!

Jesus kaies käz wechsakänechtamon nenach io, sawänemena.

Jesus kaies tachpänäion anamihäwachtiko nenach io, sawänemena.

Jesus kaies tachpänäion anamihäwachtiko nenach io, sawänemena, kiseha: mawena.

Jesus, Marie, Joseph,

Ki pakitänamon

Neall, netäh, netätchiak.

Ne pämatesin miniu.

Jesus, Maric, Joseph,

Mächno kanawchekor.

Katäu iskoanamion.

Jesus, Marie, Joseph,

Ishiasekon, asau

Wanachkion, as kes

Nepäion achkihe. Amen. 


\section{MAMTACHTOZEKAN}

mawau käz achpechtänetakositoa kesekoch i $7 \quad$ äp toa asau keketotamawakichtoa waiesakesit io.

Täpänemeach, sawänemeach! Jesus Christ, sawänemeach! 'Täpänemeach, sawänemeach! Käz Marie, keketotamowin! Mawa! käz achpechı̈̈netakosiäk asäniwiäk, käz asäniwiäk, keketotamochkon!

Käz Abel, keketotamowin!

Mawau käzachpechtänetakosiäk; keketotamochkon!

Käz Abraham, keketotamowin!

Käz Joan Baptist, keketotamowin!

Käz Ioseph, keketotamowin!

Mawau käz achpechtänetakosiek käios kaies pis sochkanamihaiäk, keketotamochkon!

Käz Pien, keketotamowin!

Käz Paul, keketotamowin!

Käz Andre, keketotamowin!

Käz Joan, keketotamowin!

Mawau käz achpechtänetakosiäk nätom 
kaies pis Mächkotächkoniäwäk, kaies äsichzikäiäk miniu mächno azimon, keketotamochkon!

Mawau käz achpechtänetakosiäk Täpänechzekät kaies kächkenohamonäk, keketotamochkon!

Mawau käz achpechtänetakosiäk nitchionächsok Täpänechzєkät kaies io nänichkäiek, keketotamochkon!

Käz Stewan, keketotamowin!

Käz Laurent, keketotamowin!

Mawau käz achpechtänetakosiäk anamihan kaies io nänichkäiek, keketotamochkon!

Käz Silvester, keketotamowin!

Käz Gregor, keketotamowin!

Käz Augustin, keketotamowin!

Mawau käz achpechtänetakosiäk, kaies käz Mächkotächkoniäwäk kaies sochkanamihaiäk miniu, keketotamochkon!

Käz Benedikt, keketotamowin!

Käz Franzis, keketotamowin!

Mawau käz achpechtänetakosiäk, kaies mamawau pematesiäk, kaies pikoatachkamik miniu kaies pemateseiäk, keketotamochkon. 
Käz Marie Magdalen, keketotamowin! Käz Lucia, keketotamowin!

Mauwau käz achpechtänetakosiäk, kaies kekachkoiäk, kaies sekawäiäk miniu, keketotamochkon!

Mawau käz achpechtänetakosiäk kesekoch äpiäk, keketotamochkon!

Kitämakänemeach; kon käkoch, echpänasin, Täpänemeach !

Kitämakänemeach, miakonamoin, Tapänemeach!

Kitämakänemeach ; otachpenin, Täpänemeach! -

Ki nächkosin; miakonamowin, Täpänemeach!

Maze nepän; miakonamowin, Täpänemeach!

Kakik werhsakänechton anamachkiach; miakonamowin, Täpänemeach !

Kàik mätä; miakonamowin, Täpänemeach!

Maze hawätok tatachkesin; miakonamowin, Täpänemeach !

Kaies pis ochtateseon; pemazehin, Tä. pänemeach! 
Kaies sachsakachkohokäon anamihäwachtiko, as kes käz kotakechtawon miniu; pemazehin Täpänemeach !

Kaies nepäion, kaies nekuahokäion miniu; pemazehin Täpänemeach!

Kaies käz apiskaion; pemazehin Täpänemeach!

Kaies mamachkatänechtakoseon, kesekoch as kes ision; pemazehin 'Täpänemeach!

Kaies pis enäsehat Waieskesit Hawätok, asau sochkiţähäskaweamä; pemazehin Täpänemeach!

Tepachkonikäu kesekach; pemazehin Täpänemeach!

Päzetatoa; - Ki pakichsenemekem asau pächtaweach!

Kon kotäu asau echpänasit; - Ki pa. kichsenemekem, asau pächtaweach!

Täpänemeon; sawänemeach!

Jesus Christ; sawänemeach!

Täpänemeon; sawänemeach !

Matchiach omos achkihe, kinach änamihaion, o wichswone Hawätok Wäwochneme, mesas käkoch nähechtok, kaies osehe; 
misiktä o wichswone Jesus Christ, pematesit Hawätok Wägiseon, kinach kaies io kotakechtok; o wichswone Waieskesit Hawätok, kaies käz achpechtänetakosehe o wichswonowa miniu asäniwitoa, käz asäniwitoa miniu; o wichswonowa miniu mawau käz achpechtänetakositois. Nas tä iochpe as kesekach pichtikaweon kakik onanekosine.

Hawätok nähe sawänechzekäion, Hawätok käshiateseon, kinach kisehamawatoa kwaiachk aianinichsitoa, ki pakichsenemekok asau sawänemochtawatoa eno katäu anich nächnit, asau kisehamawat miniu mawau o päzetonon.

Ne pakitänemon Hawätok Keau, achkihe kaies io osechzekatäk, kes nepäon achkihe asau nasächkawazin Hawätok kaies osehe. Nas tä kat ki miachkiakok käz asäniwak kitätchiakon! Nas tä kat käz kächkenohamakanok ki miachkiakok! Nas tä anich witchiwatoa käz achpechtänetakositoa anamihan kaies io nänichtakoa! Nas tä kat kit äshianekok kaiäz kekachkowitoa! Nas tä kat ki wanachkim 
enis asitoa käios kảes pis sochkinamihatoa! Nas tä Jesus Christ kat ki somichkitak; nas tä miniu kat ki konikapawehek enis asitoa käz achpechtänetakositoa! Nas tä kon kat ki witchiwawak kakik kiskanitipächkato äsiatoa, kakik iskotia ächtanächnäsitoa! Nas tä Mäz Hawätok kat ki mowinesehamak maze hawätokon! Kachpe anipiatäion, nas tä kat osemowak maze hawätok isiatoa kakik wanitipächkato. Nas tä ki Mäz Hawätok kat nipitau asau mewenesehazin saichkanächkoa! Naskat au osemowak saichkanatoa Mäz Hawätokon! Winua tä maiächno isikitoa au onanikinichtamukänanametotHawätok. Nas tä mawau maze hawätokok kat tikisewak, nas tä miniu Mäz Hawätok kat kinuakok kon kotäu asau echpänanächkoa kesekoch echpe ishion! Nas tä Jesus Christ, kaies sachsakachkoho kinach io, kat ki miakonamak wechsakänechtamowin, nas tä miniu ki miakonamak kakik nepän enoch kaies io nepäion! Nas tä Jesus Christ, pematesit Mäz Hawätok Wägiseme, kat kit anik kesekoch! Nas tä kat ki miako- 
namak ki päzetonon kat kit anik tä otäniniane maiakonapamichtoa! Nas tä ki sawänechtakosim näwat kaies akuachseme, kakikächkamik miniu asau weg asion! Nas tä kat ki sawänechtakosim kakik kesekoch asitoa sawänechtakositoa mächno hawätok.

Ki pakichsenemekem, Täpänemeach, kat asau sawänemat eno kätau anich pematesit kaies anochkitok asau achnot enis au tasi achpänemit kesekoch asau isiat. Amen.

Ki pakichsenemekem, 'Täpänemeach, asau sawänemat otätchiakon anamachkiach kat asau isianit. Amen.

Ki pakichsenemekem, Täpänemeach, asau osimihat otätchiakon, kaiesis osimihatoa Enoch misik tä Elias enis nepäne. Amen.

Ki pakichsenemekem, Täpänemeach, asau osimihat otätchiakon, kaiesis osimihat Noä, as kes moskenä. Amen.

Ki prkichsenemekem, Täpänemeach, asau osimihat otätchiakon, kaiesis osimihat otätchiakon, kalesis osimihat Abraham as mäk Kaldäu inäniwak. Amen. 
Ki pakichsenemekem, Täpänemeach, asau osimihat otätchiakon, kaiesis osimihat Job mawau wechsakänechtamowine, mawau miniu sänakach. Amen.

Ki pakichsenemekem, Täpänemeach, asau sawänemot otätchiakon, kaiesis sawänemot otätchiakon, kaiesis sawänemot Isaak, kat as kes paketenatoa ochnon os pos nänekot paketenekan io. Amen.

$\mathrm{Ki}$ pakichsenemekem, Täpänemeach, asau osimihat. otätchiakon, kaiesis osimibat Lot iskotia, as kes wenotek Sodom. Amen.

Ki pakichsenemekem, Täpänemeach, asau osimihat otätchiakon, kaiesis osimihat Moses, Pharonon as katäu maze echpänanekot. Amen.

Ki pakichsenemekem, Täpänemeach, asau sawänemot otätchiakon, kaiesis sawänematoa näniu oske inäniwak, kishiopächkishigone kat asau winositoa. Amen.

$\mathrm{Ki}$ pakichsenemekem, Täpänemeach, asau osimihat otätchiakon, kaiesis osimihat Susana, as kes penächseme. Amen.

Ki pakichsenemekem, Täpänemeach, 
asau osimihat otätchiakon, kaiesis osimihat David, as katäu maze echpänanekot Saulon misik tä Goliaton. Amen.

Ki pakichsenemekem, Täpänemeach, asau osimihat otätchiakon, kaiesis osimihat Pienon misik tä Paulon kipachkoahotiwikamiko. Amen.

Kaiesis osimihat kaiekachkoit käz Tekla anamihan kaies ochzeane wechsakänechtamowene, ene tepach au isis sawänemot eno kätau anich pematesit, kakik sawänechtakosine kesekoch tä asin. Amen. Kaies nepäk tä enoch kaies wechsakesit, ene iom pos ätamä papik neu.

Pis wetochkohokon mawau asäniwiek! Pis nachkiskochkon, mawau asäniwiek! Otachpenächkon otätchiakon miniu paketenamochkon Täpänechzekät. Nas tä kat otachpenik Jesuson, nas tä miniu asäniwon kat isianik enis as tase wanachkit! Nas tä kat otachpenäwak otätchiakon asau wanatoa änanametot Täpänechzekänit.

Kakik wanachkione asin, Täpänechzekäon,

Kakikächkamik miniu wanenamowin. 
Nas tä kat anäwak asäniwak änanametot Täpänechzekänit.

Täpänemeon, sawänemeach. Jesus Christ, sawänemeach. Täpänemeach, sawänemeach. "Nochnenau kesekoch äpeon". Kit anomächkon, Marie".

Ki pakitänamakäm, Täpänemeach, otätchiakon eno kaies nepäk, asau wez pematesit. Ki pakichsenemekem miniu, Nochno nähe sawänechzekäion, Kikis Jesus ki io pakichsenekem asau kisehamawat mawau o päzetonon, kakik wanachkione asau wanot. Amen.

Kakichkotakan asau kächkinachkoa änamihatoa au isichziketoa, wäwänin asau sawä\#ematoa anino kaies näpikoa.

Kes ani nepäk weiak pos äkit posächkotänau weau, pos pechnahau tchepai mächkachko äsis wichkichtok wepoz. Kon pos kanawehanon kinis näniu konakach. Kespin maze esetot nanas nepäk, kespin miniu kon aianinichsit, kespin kon nekoz nekotono paketcheket tachnäno 
pepon Pachk as otächzechne, pon paketänemächkon weau enis Katolik änamihatoa. Echpo otätchiak winosit kakik anamachkiach weau maze pematesit kon enis pos atawon mamawau änamihatoa, kes hewak Käz Mäz Mächkotächkoniäwak Mäz Hawätok o wichswone. Enechpe weau tasit weke, nekot amo peme wasachkonenegon sikahapuatäu pos sakächnatäu achpäniu kächze weo. Ene tä pichtiketoa pos papik otchichtchikoanetawak, pos anamihätawäwak miniu, Hawätok asau sawänemazin otätchiakon. Ene tä kon kinis pos ot apinowawon, pos kanaponäwak. Kon weiak metämoch pos otinon wanitipächka. Ene nesik nish konopoz näniu inäniwak, mächno änamihatoa, pos tanäsewak. Akiko inäniwak kon poz o käz kikitinowawon, kon miniu ot aiäninowawon. Konopoz enoch näpik käz kotakechtau kishi chnasoane. Neanekotochkin pos anamihäwak otätchiakon io asau wepoz onanekosit kesekoch Hawätok weke.

Mawau pos witchiwawak äsis wichkich- 
tokoa echpe änamihatoa pitokoa weau anamihäwigamiko.

$\mathrm{Ki}$ käz mächno esetom as kos keketotamowatoa kaies nepäkoa, Hawätokon asau sawänemekotoa, wepoz miniu asau otachpinikotoa kesekoch. Nas tä käz kon asau wesakänechtachkoa kiskichnasoane, ene au is enänematoa wawepoz. Kätin iachpez wesakänechtamuk kishichnasoane äpitoa, anech tä opäsik nemewach, anech tä opäsik mänawaz. Kon weiak pematesit achkihe pos o wichkichtonon asau kächkenach äsis wesakänechtachkoa kishichnasoane äpitoa. Kon wesakänechtamoan atäwon omos achkihe, kishichnasoane $\ddot{a} \vee$ is takik. Kinis miniu wesakänechtamuk $w$ anech, nemexkach tachnäno pepon kotakechtawak. As mäk käz wesakänechtachkoa, änєkochkitähätoa ta panäwak Hawätokon, wenach änänechtach naskat au esekit, achpäniu enänechtomuk. Iachpez sasipänochtamuk ächpez wesakänechtachkoa. Nekotäs nenau pichtikem kesekoch, ene änänichsitoa; nas tä wepoz kat nenäwau Hawätok täpanak, achpäniu enänech- 
tamuk, ene tă ene wä kotakänichtachkoa kon as wichkichtokoa asau näwatoa Hawätokon au ächpez tanäsitoa kishichnasoane.

Kätin iachpez kwaiachk tepachkonikäu Hawätok, au käz kotakehätr miniu anino käkiskamenik päzetonechson, kes ani pematesenit achkihe. Eno opäsik mäse käkiskach päzetonechson, opäsik miniu au kotakehau; eno tä opäsik mänawaz kakiskach,opäsik mänawaz miniu pos kotakehau Ene kätau io maze esetaion mänawaz. Poz päzetonechoon käz sechkatonon $\mathrm{Ha}$ wätok, kinau io kotakehekonau miniu.

Tachnäno kesekach keketotamowin kishichnasoane kotakichtokoa. Konopoz kotakänechtomuk kenepopanik. Keketotamowın, pon miniu nekotoch wanänemin. Ki kes pemehekok, ki kes kanawehekok miniu, ene au io keketotamowatoa achpäniu, Hawätok asau sawänemekotoa, wepoz miniu asau otachpinikotoa kesekoch. Eneko au keketotamowawak ki nekehekonawak, kinitchionechsenawak, kit änawämakanerawak, mawau miniu näpikoa. Jach- 


\section{0}

pez peponächkuchkin pos ki io akemawak anamihämiguk, kinach miniu ächtaseäk koko. Wäwänin asau wetochkawatoa,

$m$ akemin anamihämiguk tach ${ }_{n}$ äno kesekach äsis wichkichtawon eneko kätau pemateseon, mämik ächpez Käz Paketenekon, misik tä pemochne kotakihiswon mihikon, konau enänechtach: naszeau Hawätok sawänemäu kishichnasoane kotakechtonik, mämik tä ne nekehekok, näuächsok, nächsemächsok miniu. Neanekotochkin anosewänon Käz Paketenekon winua io. Ano pos nekami näpikoa wä Käz Paketene.

kon, konopoz miniu echpe Mächkotächkoniäu as paketänemazin.

Näpikoa sawänenıin, Jesus, kesekoch asau onanekositoa isis paketenamowin, kinach Jesus käshiateseon!

$\mathrm{Ki}$ papamächtakäm, ki tapanakäm, ki mänazehekäm, ki mamiawamekäm.

Nas tä kat ki mächno nochtawemenau, näpikoa tepänemin!

\section{Libera.}

Täpänemeon, pon achpakesena kakik iskotia, käz säkeseu kesekach. 
Enechpe kesik au nänächkiskak, achkäu miniu asau nänächkiskamakach.

Enechpe pis tepachkonatoa mawau pämatesitoa, iskotäu asau atawon.

Iachpez neu kätin ne nänächkiskam, ne säkisem miniu, echpe asau otächzechne tepachkonikan, Mäz Hawätok miniu o nächkosin.

Enechpe kesik nänächkiskak, achkäu miniu asau nänächkiskamakach.

Enechpo au otächzechne tepachkonikan, Mäz Hawätok au käz säkeseu kaies maze esekesinit tä au käz anemesewak; au käz säkeseu kesegct, au käz wesakänechtamowin kesegot miniu!

Enechpe pis tepachkonatoa mawau pämatesitoa, iskotäu asau atawon.

Täpänemeon, kakik wanachkione kishichnasoane äpitoa; kakik wasinakwot kesekoch.

Täpänemeon, pon achpakesena kakik iskotia, käz säkiseu kesekach.

Enechpe kesik au nänächkiskack, achkäu miniu asau nänächkiskamakach.

Enechpe au pis tepachkonatoa mawau pämatesitoa, iskotäu asau atawon. 
Jesus Täpänemeach, nähe sawänechzekäion, mawau kishichnasoane äpitoa kakik wanachkione asin. Amen.

\section{Dies Irae.}

Au nächkosiu kesegot ani achkewik, au anemesimakot; achkäu au wenotäu.

Iachpez neu au käz säkisewak mawau pämatesitoa echpe au näwatoa Jesuson asau pianit asau pis tepachkonenach.

Pipikoan au kaketowäu, mawau näpikoa au nochtamok: ene tä mawau asau ishiatoa asau tepachkonichtoa.

Maฬ̂au näpikoa au mamachkatänechtomuk echpe au apiskatoa: asau nachkomatoa an topachkonikotoa.

Masenachigon au pichtikatäu, mawau asasatäk: au ochtänikatäk asau tepachkonikäiach.

Wnis au mesächse uu tepachkonikät, mawau kaies kiazekatäk au kächkinakoatät: ene tä Hawätok asau wasechtawahazin mawau kaies maze esekesinit.

Ta tä au isis nachkomake nenach kaies käz päzetaion? Hawäne enoch au keketo- 
tamo? Pochz kwaiachk kaies pematesitoa au käz säkisewak.

Jesus, kätin ki kohnin; ano tä ki sawänemim: kit änänemim asau pemazeheion, nähe sawänechzekäion!

Mächkawänechtach, Jesus, nähe sawänechzekäion! ki kes pis io ishiom ios achkihe, usau pemazeheion: pon achpakesena kakik iskotia echpe au tepachkoneion.

Ki kes käz kotakechton achkibe as kes pemazeheon; ki kes sacbsakachkohokäm anamihäwachtiko nenach io: nas tä nap kat ne mächno enapazechton kaiesis käz kotakechtawon.

Jesus kwaiachk au isis tepachkoneion, kisehamawena onekoch kaiesis jäzetaion: konomächtchiu asau otächzechne echpe au tepachkoneion.

Ne käz koskänechton as kes päzetaion; iachpez neu net äkisim mächkawänechta monin no päzetonon: Hawätok ki mamiachtumin asau ponikitätaweon.

Ki kes ponikitätawau Marie Matenos, ki kes ponikitätawa kaies käz kimotäch- 


\section{4}

kit: kit äsis pakichsenemin tä, misik tä nenach asau ponikitätaweon.

Kon ne täp achpechtänetakosenon os pos ponikitätaweion: kinä tä, nähe sawänechzekäon, kit äsis mamiachtomin, pon kakik iskotia achpakesena.

Pon ki nämachtchione asina, asan wanatoa kries maze äsikitoa: kit äninionech tä asina, asøu wanatoa kaies mächno äsitatoa.

Echpe au mewinesihatoa kaies maze äsikitoa kakik iskotia: nenach tä natomena as dase natomazin Kochne Hawätok täpanazin.

Ki mamiachtomir as koskänechtamon, as aninichsion änekochkitähäion: echpe au nepäion sawänemeach.

Echpech au otächzechne asau apiskatoa mawau pämatesitor, mawau au käz mawak: Hawätok au tepachkonäu kaies nëchkehekot. Nasotä kat ponikitätawa. wak.

Jesus Täpänemeach, nähe sawänechzekäion: mawau kishichnasoane äpitoa kakik wanachkione asin. 


\section{Miserere.}

Sawänemenena, Hawätok: kinä näbe sawänechzoliäon!

Kinä kaiäz nähe sawänechzekäion : ki sehamawena no maze esekesin.

Ne maze esekin achpäniu kisechneiapawatowena; kisehamawena tä miniu ne päzetonon.

No kächkenơ⿱ ne maze esekin, ne päzeton miniu kakik ne nämin.

Kinä nesik kaies nächkehenon, ki kes m näxim kaiesis päzetaion : sawänemena asau papamächtakäion: kinau nochkehawak änochtachkoa.

$\mathrm{Ki}$ nä kächkenamon, päzeton ne kes nanawehekom: konomächtchiu as mamazetaweion.

Ki mäninichton mächno esekeseu ona. muon miniu: ki kes kächkenohamawin, asau nipuachkäion.

Säsopinama sikahapuasena nenau penesim : kisechneiapawasena, opäsik tä kohn äsis wapiskenit, nenau isis wapiskinim.

Wechtamawena asau sawänemeon, iach. 


\section{6}

pez tä au mächnochtakwoton nechtawakon: nechkanon miniu au onanekosimakaton.

Pon zenopachtach ne päzctonon: mawau miniu kisehamawena ne maze esetonon.

Iazichtowena netäh asau mächno esetahäion: sawänemenach tä miniu asau nipuachkäion.

Pon pakesena änanametaion, pon miniu miakonama ki sawänechzekan.

Pemazehena, nenau onanekosim kinach io : sochkänichtamihena, mesena ki sawä. nechzekon äsis tatachkesimakach.

Nenau kächkenohamawak päzetatoa kit äsechzekan : ene tä maze pämatesiroa asau aninichsitoa.

Pemazeheua tä miniu kisehamawena ne maze esetonon, Hawätok Täpänemeon, nähe sawänechzekäion : net änaneu tä kinau ohohemekom äsis käshiateseon.

Hawälok Täpänemeon, tawanonena : neton tä kinau mächno wawechnekom.

Kespin paketenekan mininichtamon, pos 
ki kes menin: kon tä ki mäninichtonon waienotek paketenekon.

Paketenekan Hawätok täpatach, ene otäh aianinichsimakach: Hawätok, kon pos ki näwanon otäh käskänichtamimakach, täpachnenichsimakach miniu.

Jesus Täpänemeach, nähe sawänechzekäon: kishichnasoane äpitoa kakik wanachkioneasin. Amen.

Anamihan ätamä echpe päkitänemeshzin mäz aia:

Jesus nähe sawänechzekäon, sawänemin mawau näpikoa, kesekoch asau onanekositoa. Sawänemin tä miniu enoch kaies nepäk, kaies paketänemake ios; kisehamawin eneko kaies päz esetot. Ki kes enänechton, Jesus: mawau pämatesitoa nenau sawänemewak; naskat nenach kat ne kotakechton, naskat anamihä wachtiko kat ne näpin, winua asau akuachsemakoa au onamichtawitoa. Ene kä kaies enänematoa, Jesus käshiateseon! Änekochkitähät ki kes onamichtak enoch kaies nepäk, ene au io sawänemat, Jesus Noch- 


\section{8}

nenau. Kisehamawin eneko kaiesis maze esetot; pon ki nämachtchione asin, as tase anatoa kakik iskotia kätau achpakinatoa, kit äninianech kä asin, as tase anatoa sawänechtakositoa, kesekoch äshiatoa.

Jesus Täpänemeach, sawänemin mawau kishichnasoane äpitoa; akuachsemin äsis kotakänichtachkoa; kakik wanachkione kesekoch asin!

Nas tä kat ki mächno nochtawemenau näpikoa asau tepänematoa. Amen.

Ene tä pos wechtach nänino "Nochnenau," "Kit anomächkon."

Anamihan ätamä echpe paketänamechzin nitchion.

Hawätok Täpänemeach, ki mamiawamekem kaies enänemat enoch nitchion, asau sawänechtakosit, kesekoch asau ishiat. Iachpez neu iochpe penesewon otätchiakon: kon käkoch o käkiskanon maze esekin, iachpez neu kätin onanikinechtam kesekoch. Änekochkitähäiach ki mamiawamekem Hawätok, Nochnenau, nähe sawänechzekäion! Ponikitätaweamä kaiesis pä- 
zetaiach; sawänemeach asau mächno esekeach, asau wichkichtawach miniu nenach nap kesekoch asau onanikinechtamach. Amen.

Nas tä kat ki mächno nochtawemenau näpikoa asau tepänematoa! Amen.

Nänino "Nochnenau," Kit anomächkon, Marie, mawau kishichnasone äpitoa io.

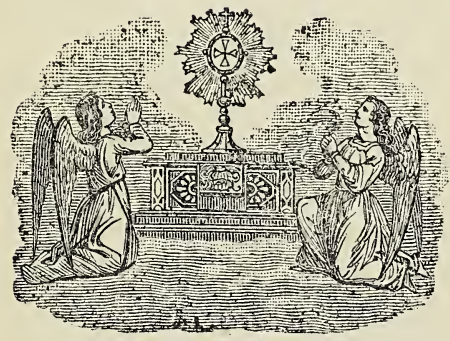




\section{VII.}

\section{MÄCHKOTÄCHKONI ̈̈WIN.}

Kakichkotakan.

Hawäne maiene ene Mächkotächkoniäwin?

Eno kä nätawänechtach asau Mächkotächkoniäwit.

Hawäne tä waichkichtok asau mikit Mächkotächkoniäwin?

Mäz Mächkotächkoniäu kä resik?

Wäki tä äs minechtoa Mächkotächkoniäwin wätachpinachkoa?

Ene ka äs minechtoa Hawätok o sawänechzekon, asau wichkichtokoa asau käz anamihatoa, kwaiachk miniu asau wichtamawitoa Jesus ot Anamihan, asau kisehamawikitoa miniu päzetonon päkizekächkin, mawau miniu wäwänin asau isitatoa, Mächkotächkoniäwak äsis natawänechtakositoa asau esechzeketoa.

Eneko kaies nanakatawänechtamon anino Käz Sawänechtakosinon, mawau änamihatoa au otachpenamuk; ene änänech- 
tach Jesus ene kä, Sikahapuanitwon, Sochkitähäskatwon, Paketchekon, Käz Ostiwin, Anamihäu Peme miniu. Mawau änamihatoa asau otachpinachkoa anino Sawänechtakosinon, ene kaies io osechtok Jesus.

Misik tä nish kes osechtau, anäch nesik änamibatoa otachpinachkoa, kon mawau; ene kä, Mächkotächkoniäwin, Anamihäu Wikichtwon miniu.

Akiko wätachpinachkoa Mächkotächkoniäwin, kon o wichkichtonowawon Anamihäu Wikichtwon asau otachpinachkoa. Akiko tä wätachpinachkoa Anamihäu Wikichtwon, kon pos o wichkichtonowawon Mächkotächkoniäwin asau otachpinachkoa, au ächpez wikimatoa metämochson.

Täpänimenach Jesus metatach nish ene inäniwon papik nätom kes natomäu asau papam witchiakot as papam kakichkotakät. Ene kä akiwak nätom kaies pis Mächkotächkoniäwitoa, kaies Mäz Mächkutächkoniäwitoa miniu. Koneu tä nekotäs misik nohekon metatach nish ene inäniwon kes onapomäu, kes enäsehäu tä 
asau kakichkotakänit. Akiwak kä kaies Mächkotächkoniäwitoa, kon o kes Mäz Mächkotächkoniäwinowawon.

Akiwak kaies enachkonikät wenach Täpänemenach Jesus; metatach nish ene Mäz Mächkotächkoniäwon kes ahnäu, nohekon metatach nish ene tä wächzetau Mächkotächkoniäwon. Nekot tä Mächkotächkoniäwon iachpez menä kes uhneu, kes iachpez menätamechkehäu Anamihane, ot Anamihäwigamiko. Ene kä käz Pien (Peter), aniwon kaies iachpez menätamechkehazin. Hawätok o masenachigone asachzekatäu ene, kaies eta Jesus enechpe, as kes menätamechkehazin Pienon. Iom kä kes enäu: "Pien, asepäh kit awim, ene tä ene asepächko asau osechtawon net Anamihäwigamiko; kon tä nekotoch pos panazechzekatäwon. Pochz wenach maze hawätok, mesas käkoch pänazechtok, kon wenach net Anamihäwigamik pos o panazechtonon." (Matt XVI. 18.)

Ene kä kaies eta Jesus käz Pienon; ene tä iom kätau eta: Pien, asepächko kit 
äsis sochkekapowin, ächpez moskawänechtamon as anamihaion. Kinach tä ki kanawechtänin net Anamihäwigamik achkihe, (ene kä, kwaiachkichine Anamihan,) kinach nätom asau kanawechtawon, kon nekotoch asau panazechzekatäk.

Ene enis kaies iachpez menätamechkehazin Pienon.

Misik tä nekotäs kes enäu : Pien, kinau menin kitchiapächkachekanon, asau pach. kinamon, asau ki pachkohamon miniu okemawin kesekoch. (Matt. XVI. 10)

Ene kä-enis misik iachpez menä as kes ahnazin Pienon ot Anamihäwigamiko, ot okomawine. Eno täpänechtach wekiwam, wenach kanawechtau kitchiapächkachekonow. Ene tä Jesus kaies menazin Pienon kitchiapächkachekanon, asau iachpez menätamechkehazin anamihane.

Ene kaies änachkonikät Jesus; ene tä ene mitchiniu äsikimakach enis kwaiachk Jesus ot Anamihäwigamiko, ene kä Katolik Anamihane. Nekoneneu enis aweu mamawau naikanesit Käz Mächkotächkoniäu, naiapiskawazin käz Pienon. Käz 


\section{4}

minikan enis, Rom äs wechtäk. Ene as tanäsit käz kinis käz Pien. Mitchiniu wenach nänino metatach näniu ene pepon kes pemateseu omos achkihe, kaies ani pematesit Jesus. Mesewäu tä kes papam kakichkotakäu, nanas as kes piat minikane Rom . Enis tä nishino metatach nianon ene pepon kes tanäseu; Konau tä miniu mawau Anamihan änekochkoa achkäu kanawechtau. Ene tä miniu enis as kes tachpänät. Koneu tä kaiäneseu kes ahnau kaies napiskawazin Pienon. Kaies nepäk enoch tä, misik kaiäneseu kes ahnau; ene tä achpäniu nanas iochpe. Achpäniu enoch Käz Mäz Mächkotächkoniäu Rom kaies tanäsit, kes iachpez nikaneseu nanas iochpe; kakik tä miniu au esekimakat, nanas asau ani achkewik; kes hewa kä Jesus: Kon nekotoch au panazechzekatäwon net Anamikäwigamik. (Matt. XVI. 18.)

Ene kä mamawau naikanesit Käz Mächkotächkoniäu, käz Pienon naiapiskawazin. Nekoneneu aweu omos achkihe. Mesewäu tä änekochkoa achkäu käz minikane tanä. 
sewak Mäz Mächkotächkoniäwak. Winua tä napiskawäwak anino metatach nish eno inäniwon, nätom kaies Mäz Mächkotächkoniäwinit. Winua menätamechkewak anamihäwigamiko, nätom winua kanawechtawak Anamihan achkihe. Mäse kanawechtau nekot Käz Mächkotächkoniäu achkäu, as tase kanawechtok Anamihan; Mäse Mächkotächkoniäwon tepänemäu. Mesewäu tä tanäsewak wächzetau Mächkotächkoniäwak; ene tä naiapıskawatoa anino nohekon metatach nish ene inäniwon, nätom wächzetau kaies Mächkotächkoniäwinit.

Wenach 'Täpänemeach Jesus mawau ene nätom kes enachkonikäu. Wenach täpänemenach Jesus mawau ene nätom kes enachkonikäu. Wenach tepenau nätom kes iachpez menätamechkehäu nekot inäniwon; metatach nish ene Mäz Mächkotächkoniäwak kes ahneu, nohekon metatach nish ene tä wächzetau Mächkotächkoniäwon. Ene tä mitchiniu äsikimakach achkihe kwaiachk Anamihane, Jesus ot Anamihäwigamiko, ene kä, Katolik Anamihane. 
Ene tä enis, au io kächkenamon misik, äsis sawänechtakoseon, as enänemekäon kwaiachk Anamihane asau pemateseon omos achkihe. Katolik Anamiban ene nesik kwaiachkichne Anamihan, ene kwaiachk Jesus ot Anamihäwigamik. Mitchiniu kä tepach isinakwot, Jesus kaiesis osechtok. Nekot Käz Mächkotächkoniäu iachpez nikaneseu, Mäz Mächkotächkoniäwak tä miniu tanäsewak, wächzetau Mächkotächkoniäwak miniu.

Wäwänin tä inänemin Jesus, as kes pichtikone ot Anamihäwigamiko, kwaiachkichne Katolik Anamihane. Wäwänin inänemin, achpäniu tä wawänin kanawechtach, Mäz Mächkotächkoniäwak miniu känawichkichtoa Anamihan omos achkihe, ene kaies io osechtok Jesus ene Käz Sawänechtakosin, Mächkotächkoniäwin. Kon weiak pos o wichkichtonon asau Mächkotächkoniäwit, konomächtchii 0 . tachpenach ene. Mäz Mächkotächkoniäwak tä nesik wichkichtawak asau mikitoa Mächkotächkoniäwin. Nanakatawänechtach tä, ene tatachkesin mainichtoa 


\section{7}

akiko wätachpinachkoa Mächkotächkoniäwin.

1.) Enoch kaies mene, wichkichtau asau paketänechsit, asau osechtok Käz Ostiwin, one kä, Jesus Weau, o Mächkom miniu. Kätin käz tatachkesin! Wenach nätom Jesus Täpänemeach kes osechtau Käz Eucharistiwin, aiatchkwot as kes wechpomazin o kächkenohamakanon. Kaies ani osechtok tä wenach, kes menäu o Mächkotächkoniämon, konau tä miniu kes menäu mawau Mächkotackoniäwon ene tatachkesin; winua tepa asau isitatoa; ene tä ene mainichtoa wätachpinachkoa Mächkotächkoniäwin. Konomächtchiu as otachpenach weiak ene, kon pos o wichkichtonon asau osechtok Käz Ostiwin. Respin katäu osechtok, naso]) neu esetau.

2.) Misik tä enoch wälachpinach Machkotächkoniäwin, menäu ene käz tatachkesin, asau kisihamawikit päzetonon, Jesus o wichswon asau io kisihamawikit. Wenach misik Täpänemenach Jesus kes menäu Mächkotächkoniamon, konau mawau Mächkotächkoniäwon kes menäu tatach- 


\section{8}

kesin, asau wichkichtokoa asan kisihamawikitoa päzetonon; iom kes enäu: Haw änetok au kischamawäk o päzetonon, enoch au kisehekatäk. Winua tä nesik Mächlkotächkoniäwak, ene kä, kaies otachpinachkoa ene Mächkotächkoniäwin, winua nesik kanawechtawak ene tatachkesin.

3.) Manäwak miniu akiko wätachpinachkoa Mächkotächkoniäwin, asau wichkichtokoa wawänin asau kakichkotakätoa, asau wichtamawikitoa kwaiachk Hawätok o kikitoanon. KKon mawau akiko änänichtakoa asau kakichkotakätoa, pos o wichkichtonowawon wäwänin asau wichtamawikitoa Hawätok o kikitoanon; kon miniu mawau enanowawon asau kakichkota. kätoa. Täpänemenach Jesus kou mawau kaies pächtaknt o kes enanon asau kakichkotakänit ; o Mächkvtächkoniämon nesik, Konau miniu mawau Mächkotächkoniäwon, iom kes enän: "Papam ishiakon" änekochkoa achkäu, papam kakichkimächkon mawau pämatesitoa." Akiko nesik kaiänit äsichzikätoa, mawau kakichkimewäwak änänichtachkoa; anoz tä kätirs 
kakichkotakäwak. Katolik A namihäwig* amiko tä kon mawau änamihat pos o wichkichtonon asau kakichkotakät, eno nesik Mächkotächkoniäu. Ene tä enis misik wä kächkenamak ächpez käz achpichtänichtakoa kit äsechzekonenau, ene kä, Katolik Anamihan.

Ene anino tatachkesinon maiene eno wätachpinach Mächkotächkoniäu. Käz achpichtänichtakwoton tä anino tatachkesinon. Ene tä au io minatänimatoa wäwänin. Kespin weiak pächtawazin Mächkotächkoniäwon käkichkotakänit, Jesus on kätin pachtawäu. Kes hewa kä wenach Jesus !

Hawänetok au pächtonak, nenach nenach nepächtak. Hawänetok tä kon au püchtonak, nenach nenach kon nenau pächtakonon.

Ene kaies eta Jesus Mächkotächkoniämon; mitchiniu tä iochpe mawau Mächkotächkoniäwon tepach enäu. Ene tä änamihat au io menatänemazin Mächkotächkoniäwon, ene au ic papamächtawazin 
miniu achpäniu. Inäniwak kikitinamuk o wichkwonowawon as näwatoa kotänos Mächkotächkoniäwon. Paitonakin Mächkotächkoniäu kikua pos ki natotamowau asau sikahapuanenak kike kinitchionächsowawak miniu.

\section{NEKONENEU ENE KWAIACHKICH- NE ANAMIHAN, ENE KÄ KA- TOLIK ANAMIHAN.}

Aiäz takimakach achkihe Anamihan, kaies äz pitok Hawätok Anamihan omos achkihe, opäsik nishoak kaiänit anamihanon kes osechzekatäwon. Iachpez nätom, as mäk mitchiniu pematesitoa akiko metatach nish inäniwak kes papam kakichkotakäwak nätom, saiä echpe anäch pematesitoa kaiänit anamihanon kes osechtawak. Koneu tä nawenau misik weiak nekot anamihan kes osechtau, nanas iochpe; käz kakaiänit tä iochpe esechzekewak pematesitoa acbkihe. Tachnäno tä wätächzechnächkin kaiänit anamihan papik hewak akiko wätachpinachkoa: Ene iom kwaiachk anamihan, ene kä kwaiachkichne 
Hawätok o kikitwon. Ene mawau ätoa akiko kakaiänit änamihatoa.

Ene tä ano ätoa, kon tä ot onaminowawon. Hawätok o masenachigone asachzekatäu. "Nekoneneu Hıwätok Täpänächzekït; nekoneneu onamichtamoan; nekoneneu siliahapuanitwon". Nekoneneu Jesus Krist kaies osechtok Anamihan, nekoneneu miniu ot Anamihäwigamik, enc kä ot Anamihan. Kon Jesus kakaiänit o kes kakichkotakänon, ene achpäniu nekonenen; kon miniu anino metatach nish o käckkenohamakanon o kes enanon kakaiänit asau kakichkotakänit, ene achpäniu nekoneneu.

Mawau akiko kakaiänit änamihatoa hewak: Nenach nekanawechtomenau kwaiachk Anamihan, kwaiachkichne Esechzekon. Ene mawau ätoa; ano tä nekoneneu nesik ene kwaiachkichne Anamihan. Anä tä au isis kächkenamä ene kätin kwaiachkichne Anamihan? Kon sanalkaton ene asau kächkenamon, kespin kä iom narakatawänechtamon: Anä ene Anamihan Jesus kaies osechtok, akiko miniu metatach 


\section{$25 \%$}

nish inäniwak kaies atotachkoa mesewän achkihe? Anä ene naiätamechne Anamihan, kakik au atäu au äz anamiha, nätom. kaies osechzekatäk, kon nawenau, kon miniu omanochneu? Anä ene anamihäu tepa kaies atäu achlikihe, änikochkoa achkäu? Ene kä nesik Katolik Anamihan. Wäwänin tä nänochtach ene.

1.) Anä anino Anamihan Jesus kaies osechtok? Ene kä kit anamihanenawon, Katolik Anamihan.

Häwätok o masenachigone asachzekatäu kaiesis kakichkotakät Jesus, kaies eta, kaies esetot miniu; mawau tä ene mitchiniu kanawechtawak Katolik änamihatoa. Ano miniu winua kakaiänit äsis änamihatoa kanawechtawak anä Jesus ot AnamiWatoa, mäse tä waieskewach käkoch kes pitawak, kes paketamuk. Winua tä Katolik änamihatoa mawau käkoch mitchiniu kanawechtawak, wäwänin miniu mawaı kanawechtawak kaies eta Jesus, kaiesis küchkenohamonach. Ene kwaiachk Jesus ot Anamihan miniu.

2.) Anä ene naiätamechne Anamiban, 
kakik au atäu au äz anamiha! Ene kä Katolik Anamihan, kon käkoch kaiänit. Jesus kes osechtau ene, ene tä au äz takimakach. Kaiänit anamihanon tä mawau nawenau kes osechzekatäwon. Aiachpez misik nekot kes osechzekatäu, ene misik nekot kotänos as mochkiskach. Ene achpäniu kaies eseke pis äz anamiha. Asachzekatäu masenachigone kaies eseke; kwaiachk miniu kächkenachkoatet echpe misik nekot kaies osechtok, kächkenanawau miniu enoch kaies osechtok. Iochpe miniu aiäz pemateseach kinach achkihe, anä kaiänit anamihanon kes osechzekatäwon. Kon anino anamihanon kwaiachkichnenon, naiätamechne nesik kwaiachk Hawätok ot Anamihan, ene kä Katolik Anamihan.

3.) Anä anino anamihäu kakichkotakan, achpäniu tepa kes atäu achkihe? Ene kä Katolik Anamihan : winua nesik Mächkotächkoniäwak, Katolik Anamihan kächkinohamawakitoa, achpäniu tepa kakichkotakäwak änekochkoa achkäu, Jesus kaiesis kakichkotakät, akiko miniu metatach nish inäniwak, nätom kaies papam 
kakichkotakätoa, kaiesis kakichkotakät, akiko miniu metatach nish inäniwak, nätom kaies papam kakichkotakätoa, kaiesis kakichkotakäwak. Kon miniu nekotoch kaiänit au esekenon nanas asau anich achkewik; kon kä Jesus pos o wichkichtonon asau kenochkit; kes enäu kä o kächkenohamakanon, mawau miniu Mächkotächkoniäwon käkichkotakänit enäu: ‘Papam "kakichkimächkon pämatesitoa änekoch"koa achkäu : tepa kakichkotakäton kaie"sis kakichkotakäion; achpäniu tä kinau "witochkonämuau kon asau wanächsemeäk "nanas asau anich achkewik". Ene änazin Jesus käkichkotakänit. Anä tä au isis wichkichtokoa asau wancchsichkoa, kakaiänit asau isis kakichkotakätoa? Ene kä achpäniu tepa wä kakichkotakätoa Mächkotächkoniäwak. Winua tä nesik Katolik kakichkotakätoa, ene kä Mäch- . kotächkoniäwak, nekoneu tepa mawau kakichkotakätoa, kon akiko kaiänit äs anamihatoa. Kon wiuua tepa o kakichkotakänowawon; kakaiänit onamichtamuk, kakaiänit miniu kakichkotakäwak kenochtachtowak miniu achpäniu. 
Kespin mawau ene wäwänin nanakatawänechtamon, kinau nenau ene kwaiachkichne Anamihan, kwaiachk Hawätok ot Esechzekon; ene kä nesik Katolik Anamihan, kon kaiänit.

Kätin mamachkatänichtakwot takimakach Katolik Anamihan nätom. Käz okemawak minikane Rom kaies tanäsitoa kes käz sechkatamuk $\Lambda$ namihan, kes käz maze echpänanawak tä anamihanit. Mämichz tä enis Rom isiu käz Pien, naikanesit Käz Mächkotächkoniäu, eneko kaies tachsitoa akiko nätom ka1es papam kakichkotakätoa. Enis tä käz minikane kes kakichkotakäu, käz mäse enis ächtanächkinit kes wichkihäu asau anamihanit, käz kinis miniu enis kes kakichkotakäu nanas as kes nächne Anamihan io ; opäsik tä enis mäse anamihawak. Akiko käz okemawak Rom kaies tanachkitoa, käz kinis, ene kä nächnuak tachnäno pepon, kes käz maze echpänanewak achpäniu änamihanit, eneko kaiesis wichkichtokoa kes maże echpänanewak, käz mäse tachnäno metatach nuachk änamihanit kes nächnäwak, Anami- 
han io; opäsik mäse kes anamihawak. Kon tä käz okemawak nesik o kes sechkanawinowawon kwaiachk änamihanit, ene miniu kaiänit äs anamihatoa kaies esechzoketoa, iachpoz kes sechkanawak Katolik änamihanit; kes maze enäwak, kes maze echpänanewak miniu eneko kaiesis wichkichtokoa; opäsik tä mäse kes anamibawak, iachpez tä wäwänin kes kanawechtawak ot anamihanowau, ene kä Katolik Anamihan.

Kespin mawau ene nanakatawänechtamon, ene enis misik au io kächkenamon äsis mamachkatänichtakoa kit anamihanenawon. Iachpez tä wäwänin kinau nänochton, kon pämatesit ot anochkiwin awewon Katolik Anamihan, ene kä Hawätok ot anochkiwin, wenach kes osechtau. Kätin wenach Täpänemenach kes osechtau, wenach kes sochkechtau mesewäu achkihe, wenach kakik kes pis kanawechtau nanas iochpe as kesekach, wenach miniu kakik au kanawechtau nanas asau anich achkewik.

Änekochkitähäon tä wäwänin pos kit 
änänemenau Hawätok, as kes mene kinach kwaiachkichne Anamihan, Katolik Anamihan. Iachpez tä sochkechtoch, iachpez miniu kanawechtoch: wäwänin esetach achpäniu äsis kakichkimikäon Anamikäwigamiko, äsis nämon miniu masenachigon. Pon tä pachpis papamänechtach kaiänit anamihan: pon nekotoch ishianon as tase kakichkimiwätoa kaiänit änamihatoa; pon kinochtamin Mächkotächkoniäwak, achpäniu kä winua kinuawäwak Katolik änamihanit, kon nekotoch kaiäuit kakichkotakan asau pächtachkoa. Pon eta miniu: Nasop nitäshiom awe pächtamon ene kakichkotakan. Pon pachpis ishianon, ki känochtamo Mächkotächlsoniäu kespin ishion, ki känochtamuau miniu Hawätok kaies mene kwaiachkichne Anamihan. Kespin Mächkotächkoniäu achnau tanäsit, kon wichkichtawon tä asau ishion as tase kakichkotakät Anamihäkesekachkin, pon kotänos kaiänit äs anamihut. Keke anamihanon, enis tä päz anamihanon, iom masenachigon miniu wapachtach. Ene asau mächno esetaion. 
Kon kaiänit kakichkotakan awe pächta, pon miniu ki nitchianechsok paketenin asau ishiatoa; pon nekotoch paketenin. Eneko miniu äsis wichkichtawon kinuawin miniu kez anamihat kon asau ishiat enis. Kon kä pachpis weskewaton, Katolik anamihat kaianit kakichkotakan asau awe pächta.

Iochpe tä nanakatawänechtach äsikimakach Katolik Anamihan, anino miniu kaiänit anamihanon. Kespin tä ene wäwänin nänochtamon, kinau nämin äsis weskewach Katolik Anamihan; opäsik tä kinau tapatan, opäsik miniu kinau mamiawamau Hawätok as kes inäneme miniu kenach asau otachpenamon ene Anamihan. Ene tä iochpe au nanakatawänechtamon.

1.) Kätin sawänechtakosewäu Katolik änamihatoa ; iachpez wäwänin winua kächkenamuk as tanäsit Jesus ot Anamihan, kwaiachkichne Anamihan kaies osechtok wenach neu Jesus Täpänemenach. Kwaiachk kächkenowawak mawau Käz Mächkotächkoniäwak mamawau nikanisitoa kaies tanäsitoa käz minikan Rom, käz 


\section{9}

Pien as kes kakichkotakät näton Winua nätom enis kes Käz Mächkotächkoniäweu, mawau tä akiko kaies napiskawatoa Pienon, asachzekasowak masenachigone, achpänili kaies napiskamäwak nanas iochpe as kesekach. Winua tä kaiänit äs anamihatoa omanochneu kes pis tanäsewak, kon ochkano o kes tanäsinowawon. Ene kä kätin Katolik Anamihan ene kwaiachk Jesus ot Anamihäwigamik, Jesus ot. Esechzekon.

2.) Jesus kes onapomäu Pienon asau menätamechkenit ot Anamihäwigamiko. Iom kes enäu: "Pien asepäch kit awim, ene tä enis asepächko asau osechtawon net Anamihäwigamik, kon tä nekotoch au panazechzekatäwon". (Matt. XVI. 18.) Pienon iachpez menä kes achnäu Jesus; ene tä kakik kaies pis esekimakach äs anamihatoa. Akiko Käz Mächkotächkoniäwak kaies napiskawatoa Pienon enis käz minikane Rom, achpäniu kes menätamechkewak. Enıch Rom ächtanesit Käz Mächkotächkoniäu iachpez naikanesit; mawau tä änamihatoa menażehäwak pächtawäwak 
miniu Jesus kaies enachkonikät asau esekimakach. Winua tä kakaiänit äs anamihatoa kon o kes. kanawichtonowawon eno kaies enachkonikät Jesus, kon tä nekotoch otächtananowawon maiamawau nikanesinit.

3.) Käz Paul iom hewa: Hawätok waieskesit Hawätok kes achnäu Käz Mächkotächkoniäwon, winua tä asau kanawihatoa änamihanit, asau enachkonikätoa mawau käkoch waieskewach, au isitatoa änamihatoa asau sawänechtakositoa kakik kesekoch. Katolik änamihatoa tä nesik kanawechtawak kwaiachk Käz Mächkotächkoniäwon, kakik miniu nätom kes pis tanäwak. Winua tä kakaiänit äs anamihatoa kon o kanawibanowawon Käz Mächkotächkoniäwon. Anä ano tanäwak, kon tä kwaiachk Käz Mächkotächkoniäu otawenon. Kes wancchsenok kä, kes nekatamuk Anamihäwigamik, Jesus ot Anamihäwigamik, as tase tanäsitoa kakik kwaiachk Käz Mächkotächkoniäwak; kanawechtawak kä Jesus ot änachkonikon.

4.) Katolik änamihatoa mesas achkihe 
tanäsewak änekochkoa achkäu, tepa tä mesewäu esechzekewak. Kon kaiänit isis ot onamichtonowawon Katolik änamihatoa, ene tepa nekoneneu änekochkoa achkäu. Kätin tä kakaiänit esechnesewak, kakaiänit miniu nekamowak anamihäwigawiko, anoz miniu käkoch kakaı̈nir esetawak Katolik änamihatoa; kon tä ene kakoch awewon. Fne tä anamihäu esechzekon, anamihäu kakichkotakan, onamichtamoan miniu, iachpez nekoneneu änekochkoa achkäu; kon kakaiänit ot onamichtonowawon Katolik änamihatoa mesewäu achkihe ächtanesitoa. Winua 1 ä äs anamihatoa käz kakaiänit kätin esechzekewak, kakaiänit kakichkotakäwak, kakaiänit onamichtamuk, käz kinochtachtowak miniu mesewäı. Kon kä kwaiachkichnenon of anamihanowau.

5.) Tanäseu enoch känawechtok Iratolik Anamihan omos achkihe, ene kä maiamawau nikanisit Käz Hächkotächkoniäı Rom ächtanesit. Kespin kotänos achkihe weiak wanechse, kaiänit tä katäu kakichkotakät, kaiänit katäı onamichtach, papik wechtą- 
mowau eno känawechtok Katolik Ânamyhan änekochkoa achkäu, ene tä as azimit äsikimakach kwaiachk Hawätok ot Esechzekon. Kespin tä weiak kinochtamazin anino Käz Mäz Mächkotächkoniäwon, saketänau Anamihäwigamiko, ene kä Katolik Anamihane. Achpäniu tä tepa takimakat ene Anamihan mesas achkihe, kon nekotoch iazechzekatäwon; tanäseu k ä enoch känawechtok. Winua tä kakaiänit äs anamihatoa, enänechtachkoa kä ene äsis onamichtamuk, ene miniu äsis kakichkotakätoa. Ene minju Hawätok o masenachigon, änänichtachkoa kä isis nänochtamuk, ene tä achpäniu kakaiänit wä onamichtachkoa, ene tä kakilk as kinochtachtitoa.

6.) Katolik änamihatoa wäwänin pächtawäwak Jesuson. Eneko kaies eta, eneko miniu kaies enachkonikät, mitchiniu kanawechtawak; nanas tä asau anich achkewik achpäniu mawau kanawechtawak. Winua tä kakaiänit äs anamihatoa kon wäwänin o pächtawanowawon Jesuson. Kon kä mawau ene kaies eta Jesus, kaies 


\section{3}

enachkonikät miniu, mitchiniu o kanawechtonowawon; mäse kes paketamuk. Täpänemenach Jesus kes osechtau ene Käz Sawänechtakosin Paketchekon; kes paketamuk tä winna kakaiänit äs anamihatoa, kon mitchiniu o kanawechtonowawon. Wenach miniu Täpänemenach Jesus kes osechtau en€ Käz Sawanechtakosin Käz Ostiwin; kes ponechtawak tä miniu ene winua kakaiänit äs anamihatoa; anä tä kes iazechtawak ene Sawänechtakosin, kon ot äsechzekänuwawon kaics esechzeket Jesus, kaies enazin miniu Mächkotächkoniämon asau esechzekenit. Käz mäse miniu kaiänıt kaies eta Jesus, kaies enachkonikät miniu, kes ponechtawak, kes iazechtawak miniu. Katolik änamihatoa tä nesik mawau kanawechtawatoa Jesus o Kikitwon, Jesus ot Esechzekon.

Kespin mawau ene wäwänin kinau enänemau Hawätok as kes mene kwaiachk onamichtamon, kwaiachkichne Esechzekon, ene kä Katolik Anamihan, iachpez tä wäwänin kinau tapatan, kakik kiuau kikanawechton. 
Mächno kanawechtoch kä kätin kit Anamihan kwaiachkichne, kinau sawänechtakosim tä. Kespin Katolik änamihat wäwänin kon kanawechtok ot Anamihan, kat esetot äsis kakichkime, kätin au maze esekel, Pon tä weiak au hewa: Matät Katolik Anamihan, osam maze esekewak Katolik änamihatoa. Kon kwaiachk osenon enoch äz ene. Kätin tanäsewak mäze isikitoa, anoz anä maze esetawak kätin. Kon tä weiak pos osenon; maze esekewak anä Katolik änamihatoa, ene tä ene Anamihan, ene kä kwaiachk Hawätok ot Esechzekon. Kespin weiak wäwänin kanawechtok, kätin au mächno esekeu, kon pachpis onan maze esetanon. Mawau käkoch waieskewach kächkenohamowak anamihatoa asau isitatoa, mawau tä käkoch mätä käz kinuawawak achpäniu. Kespin weiak papamänechtach Katolik Anamihan, papamächtawazin wäwänin Mächkotächkonäwon käkichko. takänit, kätin iachpez kwaiachk eseken.

Kespin tä kon papamänechtach ene anamihäu kakichlotakan, kätin papik 
anoz an maze esckeu. Wne tä enis kächkinakoatit, kätin weskewat Anamihan.

Kespin tä änamihatoa pochz maze isikitoa, ene kä wä maze äsikitoa kon as papa. mänichtachkoa ot Anamihanowau.

Yon miniu weiak pos hewa: Kon weskewaton Katolik Anamihan, kon kä pochz Mächkotächkoniäwak mawau o mächno isikinowawon, anä maze esekewak. Kätin: käz koskänichtakwot tä ene. Kon kätin mawau Mächkotächkoniäwak o mächno isikinowawon; ano tä Katolik Anamihan käz weskewat, kwaiachk Hawätok ot Esechzekon. Kespin Mächkotächkoniäu wenach maze esekit, kon Anamihan o io maze esekenon; ene wä maze esekit kon o kanawechtonon Anamihan miniu wenach. Ene miniu nätom kaies eseke naianckotochkin; ene tä kaies io eta Jesus: "Kespin Mächkotächkoniäu kon kwaiachk esekit, pon esetach äsechtot ; äsiه kakichkotakät tä esctach," (kespin kä kwaiachk kalichliotakiat.) Täjänemenach Jesus metatach nish o kächkenohamaka. non, o Mächkotächkoniämon kes kana- 
wehäu, achpäniu kes papam witchiwäu, wäwänin kes kächkenohamowäu, mawau miniu waieskewach tatachkesin kes menäu, asau kakichkotakänit mesas käkoch. Nekot tä kes käz maze esekeu, ene kä Judas Iskariot. Pos hewa tä weiak: Kon weskewaton Jesus ot Esechzekon, kes maze esekewon kä nekot o Mächkotächkoniämon. Waninäwisit nesik pos hewa ene. Ene tepa miniu iochpe. Käz weskewat Katolik Anamihan, anä tä änamihatoa maze esekewak. Kon kä Anamihan io o maze esekinowawon; ene wä maze isikitoa kon o papamänichtanowawon Anamihan iachpez waieskewach.

\section{Anamihan.}

Hawätok Nochne kesekoch äpeon, änekochkitähäon ki mamiawamin as kes meseon Katolik Anamihan, aiano kon achpechtänechtakoseon. Ächpez kä keshiateseon ki kes mesin, as kes kitämakänemeon. Wäwänin, Nochne, kit änin. Opäsik tä wäwänin asau mamiawamenon, ne katäu mächno kanawechton net Anamihan 
1achpez kaiäz achpichtänichtakoa. Kon ne kikitoan nesik ne katäu onon asau mam awamenon, net äsetom miniu ne katäu on. Kon nasop ne katäu kanawechtonon ene Anamihan kaies meseon, Nochne käshiateseon, wäwänin achpäniu ne katäu enapazechton. Sawänemena, wetochkawena, asau wichkichtawon asau esetaion kaiesis kesänechtamon iochpe. Amen.

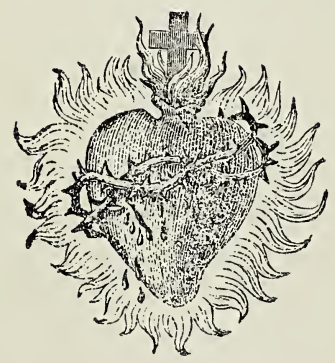


268

VIII

\section{ANAMIHAU WIKICITWON. \\ Kakichtotikan.}

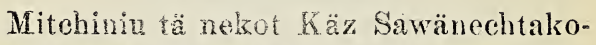
siu nanakatrwänechtach, ene kä, Anamihäu Wikichtwon. Wäki tä äsis minichtoa Anamihäu Wrichtwon echpe wätachpinachkoa? Ene kä äsie minich øa anamihäu nipawitos Huwätok o sawänechzekon, asan tapanititon, asan mächno isikitoa, asan mächno mazikiha ou onitchionächsowawon, $k$ wainchk minu asun lächkenohamowaloa anamihn.

Hawåne tä waichkichtok asan wekechtahnzin änamihanit? Mächkotächkoniäu nesik. Kespin łä weiak nasächkawazin kaiänit kakichkimewäwon, koneman misik tepachkonikäwon, käz päzeto. Eno nesik Mäz Mächkotächkoniäu pos waichkichtok asnu kisehmowazin o päzeton. Iachper matät one.

Wäki tä su ixitatoa änamihatoa konomächtchio a๕an anamihäu nipawiton? 


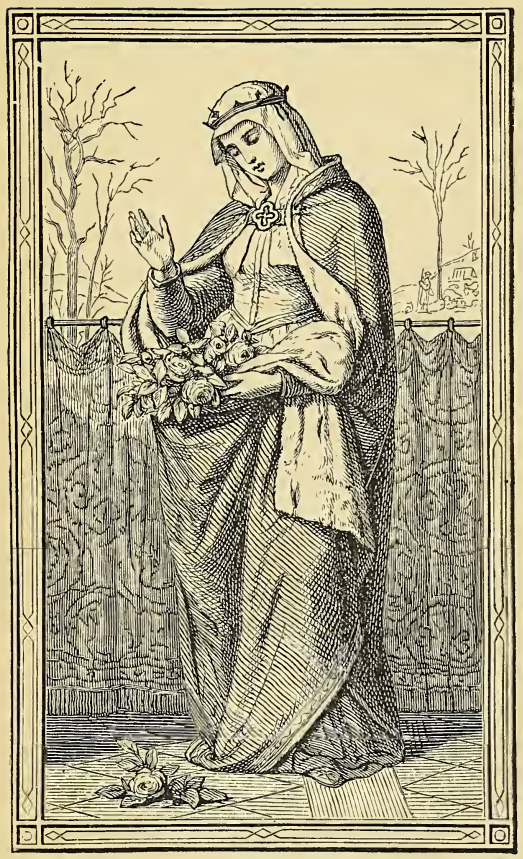

St. Elizabeth. 

Au aninichsowak $k \ddot{a}$, wäwänin miniu au paketchekewak konomächtchiu asau anamihäu nipawitoa.

Kakik kä au wekechtowak ene änänechtakositoa anamihäu nirawitoa?

Au äz pemalesiton au wekechtowak ene änänechtakositoa anamihäu nipawitoa; käz maze esetau, kespin pachkianazin waiekemazin.

Nätom wenach Täjänemenach Hawätok kes osechtau waioskowach Wikichtwon. Kes panazechtawak tä pematesitoa ach kihe, kon wäwänin o kes kanawechtonowawon. Nawenau tä wenach Jesus, megas käkoch weskewach kaies kächkenohamakonau, kes käz weskewechtau miniu, kos kächkenohamowäu ảnamihanit, wäwänin winua asau kanawechtokoa ene Anamihäu Wikichtwon, wäwänin asau isitatoa, äsis mininechtach Hawätok. Ene tä ene asau. kanawechtokos, ene wä otachpinachkoa ene käz Sawänechtakosin, Anamitäu Wikichtwon, kaies osechtok Jesus, asau minichtoa winua änamihatos kätau wikichtitoa. 
Wäki tä Hawätok kaies io osechtok Wikichtwon? Näniu kä ene kaies io osechtok Täpänemenach Wikichtwon. Wäwänin tä nenochtach ene.

1. Ene kä kaies osechtok, opäsik asau manätoa, achpäniu asau tanäsitoa pämatesitoa achkihe. Nätom kä, echpe kaies kesehazin nätom inäniwon nätom metämochson miniu, wenach kaies wekechtahazin, iom tä kes enäu: Wikichtikon, onitchianechsekon, opäsik asau manäiäk, nanas asau moskinätoa pämatesitoa mesewän achkihe. Ächpez kishiatesit Hawätok, inänechtom asau iachpez manätoa pematesitoa achkihe, mawau tä asau mächno tanäsitoa omos achkihe, misik tä kesekoch kakik asau sawänechtakositoa. Kespin tä weiak mächno kat tanäsit, kon ishiat miniu kesekocb, wenä neu taiotasit. Kon tä Hawätok o mäninechtonon, pämatesitoa anoz asau ochtatesitoa, tepach manätowak. Nekot inäniu, nekot metämoch miniu au wekechtowak, achpäniu mamawau au tanäsewak, nanas nekot asau nepäk, ene tä wäwänin asau mazikihatoa 
nitchianon, opäsik asau manätoa pematesitoa achkihe. Ene änänechtach Hawätok T'äpänemenach, ene kaies ioch osechtok waieskewach Wikichtwon.

2. Misik tä Hawätok, äsis kishiatesit, inänechtom, asau witochkatitoa pämatesitoa achkihe, ene kä inäniu asau wetochkawazin metämochson, asau mächno totawazin, asau kanawehazin, enoch metämoch miniu asau wetochkawazin inäniwon, asau pächtawazin, asau anochkitawazin eneko äsis wichkichtok; asau mächno echpänanetitoa miniu kespin wesakesit nekot, koneinau miniu koskänechtach. Jachpez nätom, konomächtchiu as tat weiak metämoch omos achkihe, iom kes hewa Täpänernenach Hawätok:

Kon weskewaton nachäs asau pematesit inäniu; ne katäu menau wekemazin tepa wenach äsinakwot. Ene tä kaies ioch osehazin metämochson, inäniu ochpekäkon kesua. Ene tä ene wä änasachzekatäk Hawätok o masenachigone: Inäniu au nekanäu ochnon, okiän miniu ; metämochson tä au witchiwäu; nekoneneu au awe- 
wak inäniu metämoch miniu, kon onau näninowawon. Ene kä kaies osechtok Täpänemenach Hawätok Wikichtwon, kakik asau witochkatitoa akiko nish, inäniu metämoch miniu.

3. Misik tä asachzekatäu Hawätok o masenachigone: Käz manät päsikoatesin. Kon asau päsikoatesitoa änamihatoa, ene kätau io tanäsit inäniu metämochson, metämoch tä inäniwon au tanäseu. Ene kä, wäwänin asau wichkichtitoa, asau iachpez wichkichtitoa, nanas nekot asau nepäk. Kon tä inäniu onau papamänemanon kaiänesewon metämochson; enoch miniu metämoch kon kaiänesewon inäniwon onaX papamänemanon. Ene kaies ioch osechtok Hawätok Wikichtwon. Kon tä weiak onau päsikoaz esekenon. Kespin enoch inäniu kon enänechtach sinawaneu asau pematesit, kon nasop metämochson onau wechnochkawanon, wäwänin zeau au wekemäu. Enoch tä metämoch kon nasop inäniwon onau keketotawanon; wäwänin au otachpenäu, asau iachpez wekemazin au äz pematesit. 
Ano weskewat Anamihäu Wikichtwon. Wenach Täpänemenach Hawätok nätom kes osechtau, Jesus tä opäsik kes käz weskewechtau. Kon tä ano iachpez ma. wau pämatesitoa achkihe inänowawon asau wikichtitoa. Winua nesik nätom pämatesitoa, ene kä, nätom inäniu, nätom metämoch miniu, iachpez kwaiachk kes enawak:

Wikichtikou, onitchionächsekon. Kon tä iochpe mawau pematesitoa ene inänowawon. Nanckot opäsık mächno esetawak kon o wekemewänowawon. Ene iom änasachzekatäk Hawätok o masenachigone: Kespin weiak wekemewät, mächno esetau (kespin kä wäwänin wekemewät;) opäsik mächno esetau enoch kon nekotoch waiekemewät. Enoch waiekechtahazin otanon, mächno esetau; (kespin kä wäwänin wechkechtahazin;) opäsik mächno esetau enoch kon waiekechtahazin otanon. Ene äz käz Paul. Konau tä hewa: Kon ki iachpez inäninawawon ene; nenach änänechtamon kit äsis kakichkiminämuau. 
Ene tä wä etamä anamihäu kakich. kotakan :

Nanekot opäsik mächno esctawak, kon nekotoch o wekemewänowawon. Opäsik wäwänin wichkichtawak asau anamibatoa, asau anochkitawatoa Hawätokon. Ene tä Mächkotächkoniäwak wä kon o wikimiwanowawon. Hawätok kä nesik winua anochkitawäwak, wenach nesik papamänemäwak, kon tä metämochson o papamänemanowawon. Winua miniu, Mächkotächkoniachkiwak änichtoa, kon miniu winua nekotoch kotänoso wikimiwanowawon. Winua kä Hawätokon nesik anochkitawäwak, Jesuson iachpez tapanäwak, kon tä inäniwon o papamänemanowawon.

Mawau Mächkotächkoniäwak, Mächkotächkoniachkiwak miniu, ene äsichziketoa, kon wikimiwänowawon; nanekot miniu änamihatoa ene inänechtamuk, kon nekotoch asau wikimiwätoa, wäwänin tä Hawätokon asau anochkitawatoa. Ene kaies isitatoa anäch miniu nätom; mitchiniu tä anäch änamihatoa ene esetawak. Kätin tä käz mächno esetawak, kespin Hawätok 
ioch isitatoa; kwaiachk tä penultesitor, nipöacbkatoa. Kätin mächno esetawak, sawänechtakosewak, ächpez en is kesekoch au mächno tanäsewak, kespin nipoachkatoa nanas asau anich pematesitoa. Jachpez. kä Jesus tapanäu paienetähänit; enis tä kesckoch winua kaies kon wikimiwätoa, iachpez kächze ioch nozpenehäwak Jesuson, oske nekamon tä nekamowak; kon tä kaiäneseu weiak o wichkichtonon ene asau nekamit, ene nesik akiko kaieś iachpez pinisitoa, kon kaies wikimiwatoa miniu nekotoch achkihe. Ene kä änasachzekatäk Hawätok o masenachigone; ene tä kätau etamä. Opäsik winua tapanäwak kesekoch, opäsik miniu onanekosewak kon kaies wikimiwätoa nekotoch, kaies iachpez penesitoa, akiko tä kaies wikimiwatoa achkihe. Kespin tä weiak iachpez kon tatachkesit asau kanawechtok penesin, kon nekotoch asau päsikoatesit, pon wenach nachäs au tanäseu. Opäsik mächno esetau weiak, wäwänin asau wekemewät, ene tä asau kon wekemewät, nasop tä asau päsikoatesit. 
Sanakot wikichtwon. Asau wichkich. tokoa tä wäwänm asau isikitoa änamibatoa waikichtitoa, ene wä otachpinachkoa ene Käz Sawänechtakosin Anamihäu Wikichtwon. Ene echpe mainichtoa:

1. Kwaiachk tatachkesin, asau wichkichtokoa sinawaneu asau wichkichtitoa, asau kon kichkachtitoa. Kätin sanakat iachpez Wikichtwon. Nekot inäniu, nekot miniu metämoch otachpenetowak asau wäwänin kächkenatitoa äsikitoa. Kespin tä kaies otachpinetitoa, achpäniu tä mamawau wekechtowak, ene tä as kächkinatitoa äsis maze isikitoa. Saiä tä iachpez wekechtowak, kon o wichkichtonowawon asau pachkinaniketoa; ene tä saiä sechkanetowak, anoz maze etowak, maze echpänanetowak miniu. Achkosik tä inäniu kaiänesewon metämochson mochnawenemäu, wenach nap miniu metämoch kaiänesewon inäniwon keketotawäu. Ene tä one kon asau isikitoa, sinawaneu tä asau pematesitoa akiko waichkichtitoa, asau ponikitätatitoa, achpäniu tä asau mächno echpänanetitoa, ene äsis minichtoa akiko 
kwaiachk wätachpinachkoa Anamihäu Wikichtwon.

2. Misik tä manäwak sawänechzekon, asau wichkichtokoa wäwänin asau mazikihatoa onitchianon, anamihan miniu wäwänin asau kächkinohamowatoa. Sanakat miniu ene. Nitchianok maze esekewak, kon o papamächtanowawon onekehekonowawon; änänechtachkoa ene kätau isikitoa. Kespin tä pakitinichtoa asau isitatoa, au käz maze esetawak, opäsik au maze esekewak, au panazehäwak otätchia kowawon ; akiko miniu wänitchionechsitoa au anemesewak tejachkonikäu kesekach, onitchionechsowawon io. Ene tä misik ene asau äsikimakach, asau wichkichtokoa tä asau mächno tepänimatoa onitchionächsowawon, asau mächno mazikihatoa, mächno mihikone asau isianatoa kesekoch enakach, ene äsis minichtoa wätachpinachkoa ene Anamihäu Wikichtwon.

Asau wichkichtokoa tä anamihäu nipawitoa, wäwänin asau otachpinachkoa Anamihäu Wikichtwon, nawaz au ani- 
nichsowak, asau pakitachkoa o päzetonowawon, asau kisihamochtoa miniu eneko kaies maze isitatoa. Ene tä nawaz kätau io nasächkawatoa Mächkotächkoniäwon, konomächtchiu asau anamihäu wikich. titoa.

Opäsik tä wäwänin iochpe nanakatawänechtach, äsikitoa änamihatos waikichtitoa.

1. Anamihachlin kä, enechpe wikichtitoa; mämik anamihan, mächno esekin miniu au papamänech:omuk, wäwänin au anamihawak. Kespin nekot opäsik tatakazit asau anamihat, tatakazit miniu anamihäwigamiko asau isiat, asau aninechso miniu wawepoz, wäwänin enoch nekot au keketotawäu sinawanäu au aninemeu. Wäwänin mamawau au anochkitawäwak Hawätokon, kukik pematesin kesekoch asau wichkichtokoa.

2. Sinawaneu an wichkichtitowak, kon onau kechkachtinowawon. Mämik sechkatänichtakwot kechkachkwon, käz sechkatom Täpänemenach. Kespin nekot katäu kechkachzekät, naskat tä eno 
nekot sinawaneu au apeu, pon käkoch kat hewa, konemau miniu wäwänin au keketotawäu waiekemazin. Kespin sinawaneu pematesitoa, mächno esetawak, mächno kächkenohamowäwak onitchionächsowawon, kespin saiä opäsik nänikinit. Kespin tä kichkachtitoa waikichtitoa, anoz miniu maze ititoa, maze echpänanetitoa, kätin käz maze kächkenohamowäwak onitchionächsowawon; käz wepoz akiko nitchianok au kächkenamuk maze kikitoanon, anoz miniu maze echpänanetwon. Mämik kätin au enänechtomuk waikichtitoa, kwaiachk asau pematesitoa, sinawaneu miniu asall wikichtitoa.

3. Au tapanetowak miniu waikichtitoa; kon tä nekotoch enoch inäniu kaiänesewon metämochson onau papamänemanon ; enoch miniu metämoch kon onau papamänemanon kaiänesewon inäniwon. Kespin tapanititoa, achpäniu au mächno echpänanetowak, kon miniu onau maze inänichtinowawon. Kespin miniu nekot käkoch kwaiachk echpänanesit, enoch nekot wäwänin sinawaneu au aninemeu 
nachäs. Kespin miniu nekot wechsakesit, enoch nekot au mächno kanawehäu waiekemazin waiesakesinit, nanas asau inänit. 4. Misik tä mamawau kakik au iwak anamihäu waikichtitoa, nanas nekot nepäk. Kespin pächkoz kaies anamihäu nipawitoa, kes kächpenawak Mächkotächkoniäwon, kon nekotoch onau wichkichtonowawon wäwänin asau pachkinanikitoa, au äz pematesitoa. Kon pochz eno weskewaton, nemewach pachkähitwon as tanäsitoa. Papik misik au na:ächkatowak, mamawau tä achpäniu au tanäsewak. Iachpez sochkon ene Anamihäu Wikichtwon; kon pachpis weiak achkihe pos o wichkichtonon asau pekonach. Mawau Mächkotächkoniäwak kon pos o wichkichtonowawon asau kächpinat kätau wikichtinit, asau iachpez wikichtahatoa. Kon tä weiak Mäcbkotächkoniäu; kon miniu weiak Mäz Mächkotächkoniäu; kon pochz enoch maiamawau nikanisit Käz Mächkotächkoniäu, pos o wichkichtonon asau pechkonach ene Anamihäu Wikichtwon. Kes hewa kä Jesus: "Iraies 
kächpetok Hawätok, kon pematesit pos o pächkonon,"

5. Wenach tä inäniu mämik aiakoamemau asau tapanazin wewon. Inäniwak, tapanächkon ki wewawak, tepa Jesus äsis tapatach ot Anamihäwigamik...... Inäniwak pos tapanäwak wewowawon, tepach äsis tapanachkoa weowawon. Hawänetok täpanazin wewon, wenach tepenau weau tapatom. Kon weiak nekotoch o sechkatonon tepenau weau; achsatom, mächno kanawechtau. Ene kaies eta käz Paul. Mämik an mächno kanawehau wewon, au mächno echjänaneu. Inäniwak, pon nächkosekon asau kechkamik ki wewak, hewa misik käz Paul. As sawänemäu miniu wewon enoch iıäniu, kon onau kotakehanon, kou miniu onau nächkenawanon, kon osam onau anochkihanon, mämik kespin nächhe wechsakesit enoch metämoch. Kespin miniu metämoch käkoch esekit, kikäskawazin nitchion, echpe mämik enoch inäniu asau sawänemäu o wewon kon iachpez asau anochkihanon, anoz käkoch sänakach 
asau osechtok. Kespin metämoch kikäskawazin nitchion, osam tä käz anochkit, konopoz nekoch au totaso, au wanechsemäu anino käkiskawazin. Kätin tä käz koskänichtakwot ene, käz maze esekiu miniu, metämoch panazehazin onitchionächson konomächtchiu as ochtatesinit. Ene tä inäniu au io sawänemazin enechpe wewon kon onau iachpez anochkehanon; wenach tä opäsik au anochkiu, asau merazehazin wewon, asau kanawänemazin. Achpäniu miniu enoch inäniu wäwänin au anochkiu, asau wichkichtok au io pematesinit wewon, onitchionächson miniu. Kon tä onau kätemakesinon, kon miniu onau menänon, kon miniu onau achtawikinon. Ke:pin ene esechzeket, ken wäwänin onau pemehanon wewon, onitchianechson miniu, au käz kitämakehäu.

6. Wenach 1 ä metämoch miniu wenach au mächno echpänaneu onapiamon, wäwänin au anochkitawäu, au papamächtawäu, miniu. Inäniu menätämechkeu, metämoch tä pos papamächtawäu onapiamon. "Kinua metämochsok, pa- 
ketenamochkon keowawon kinapiamowawak, tepach äsis paketenamo. wek Täpänemenach. Inäniu menätämechkäu wewon, tepa Jesus äsis menätämechket Anamihäwigamik. Tepa tä Jesus äsis tejänechtach Anamihäwigamik, ene tepa metämoch au isis tepänemekot onapiamon." Ene äz käz Paul; ene kä Hawätok o kikitwon. Nätom miniu Hawätok kes enachkonikäu ene; kes enäu kä nätom metämochson:

Kinau anemesim achkihe..... Kinapiam tä kinau tejänemik...... Ene tä metämoch au io papamächtawazin onapiamon. Kespin metämoch achpäniu katäu onamit, wenach achpäniu katäu menätamechket, mawau käkoch wenach katäu tepänechtach, wcnach iachpez tepänemäu miniu onapiamon, kätin maze esechzekeu, kinochtamäu Hawätokon, pekonom enachkonikät. Kespin nesik iuäniu maze esekit, katäu maze esetot tä, kaläu maze ech pänouäu wewon miniu, enechpe enoch metämoch kon onau papamächtawanon onapiamon, Hawätokon tä nesik au papa- 
mächtawäu. Asachzekatäu kä Hawätok o masenachegone :

Pämatesit achkihe opäsik au papamächtawäu Hawätokon, äsis papamächtawazin tä pämatesinit. Hawätok tä hewa: "Pon nekotoch weiak kat maze esetau." Kes. pin tä weiak omos achkihe pematesit enazin wez pematesewon : "Maze esetach;" pon kat maze esetach. Opäsik kä an papamächtawäu Hawätokon, "pon maze eseta," äkozin.

Mämik tä metämoch waiekemewät aiakoamemäu, asau mächno kanawihäsit. Kespin nekoch äsikizin, kikäskawazin kä nitchianon. Enechpe mämik au mächno kanawechtau weau. Kespin mächno kanawihäsit, käkoch au totaso tä, ene tä panazehazin nitchionon kaies ano kikäskawazin, kätin mämik iachpez käz maze esetau; iachpez kätin käz koskänichtakwot ene. Naszeau wäwänin kat nenochtamuk ene mawau änamihatoa waickemewätoa. Enoch nitchion anamiach mitchiniu tanäseu, kanawehäu saiä otätchiakon. Kespin tä metämoch käkiskawazin anino 
nitchionon, käkoch totasit, iachpez panazehäu arino nitchionon. Kon nekotoch kesekoch onau isianon enoch nitchion otätchiakon, kakik kotänos au kitämakesewon. Kon kä o sikahapuasinon enoch nitchion; nipuach konomächtchiu as ochtatesit; kon nekotoch kesekoch onau ishianon. Ene kä ene wä koskänichtakoa iachpez neu. Au käz anemeseu enoch metämoch tepachkonikäu kesekacb, kespin kon mächno kanawihäsit, ene tä ere wä panazchazin onitchionächson. Opäsik ene maze esekin, äsis maze esekeu tä nänätwon. Kespin weiak nächneu saikahapuasit, kesekoch au isiu, kespin käz päzeton kon kikäskach echpe nächne; kespin tä nitchion panazehe, konomächtchiu as ochtatesit, konomächtchiu as sikahapuasit, kon nekotoch kesekoch onau ishianon, kakik kotänos au kitämakeseu. Kätin iachpez käz koskänichtachkwot ene! Ene tä metämoch kätau mächno kanawechtok weau, kespin kikäskawazin nitchionon. Kon enechpe onau käz anochkinon; kespin miniu kisechnezekät, sina- 
waneu au tasechtau; kon käknch kosekwoa papik neu onau ispänanon; kon miniu kä $z$ mächseu wenach onau pichtikatonon; kespin minin pemochnet, mämik pepo, sinawaneu au pemochneu, kon asau pätachkisitächsä. Achpäniu kä, mesewäu miniu, au mächno kanawehäso enechpo metämoch, kon käkoch asau echpänanesit.

Naszeau mawau metämochsok waikimiwätoa wäwänin kat kanawehäsowak, kon nekotoch asau esckimakach ene, weiak asau iachpez panazehazin tepenau onitchionächson. 



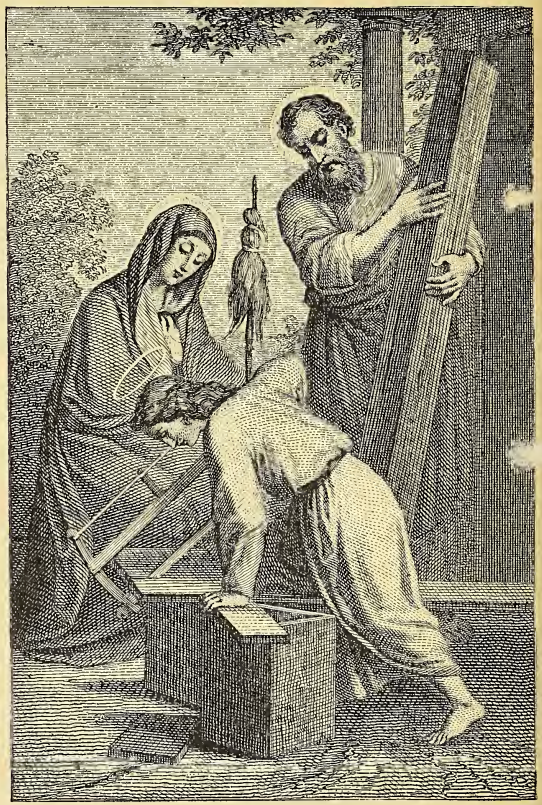

The Holy Family. 
Kakichkotakan, asau kächkinachkoa waikichtitoa wänitchionächsitoa miniu, au isichziketoa, asau wichkichtoasitoa kakik sawänechtakosin kesekoch ächtakik.

Kwaiachk asau pematesitoa waikichtitoa wänitchionächsitoa miniu, ene au isis mächkawänichtachkoa, au isitatoa miniu. Nihu tachnäno kä one, kaies io osechtok l'äpänemenach Wikichtwon, ene kä:

1. Opäsik asau manätoa, achpäniu miJiu asau tanäsitoa pämatesitoa achkihe.

2. Wäwänin asau mazikihätoa nitchianon, asau tapanatoa Täpänechzekänit Hawätokon, asau kohnatoa, asau anochkitawatoa miniu, ene tä kakik kesekoch asau sawänechtakositoa.

3. Ene neu miniu kaies io osechzekatäk Anamihäu Wikichtwon, kon asau päsikoatesitoa änamihatoa, asau pinisitoa tä achpäniu äsis mininechtach Täpänechzekät.

4. Aiatchkwot tä ene wä wikichtitoa änamihatoa, asau tapanititoa, asau witoch- 
288

katitoa, asau mächno echpänanetitoa miniu, kespin wesakesit nekot konemau miniu koskänechtach.

Jesus kes osechtau ene nekot käz Sawänechtakosin, Anamihäu Wikichtwon äs wechtek, asau minichtoa iachpez waikichtitoa Hawätok o sawänechzekon, asau tapanititoa, asau mächno isikitoa, asau mächno mazikihatoa onitchionächsowawon, asau kächkenohamowatoa miniu Anamihan kwaiachk pematesin miniu.

Waikichtitoa mamawau au tanäsewak, nanas nekot asau nepäk; kon onau pakinitinowawon sechkatom kä Hawätok pakinitwon. Waikichtitoa au tapanetowak, au menatänechtowak miniu. Hawätok käz sechkanäu päsikoatesinit, au käz kotakehäu miniu kakik iskotia anamachkiach, ene au io kon päsikoatesitoa waikichtitoa. Ene nesik kaies anamihäu waikichtitoa, ene nesik au isis wikichtitoa, akiko tä kaies iachpez wikichtitoa pon kat wekemewäwak. Inäniu kaies kon anamihäu wekemewät,' iachpez päsikoaz esetau as wekemazin metämochsok; 
misik tä metämoch, kaies kon iachpez wekemewät, käz päsikoaz esetau as wekemazin inäniwon. Sinawaneu au wekechtowak änamihatoa kaies iachpez wikichtitoa; kon onau kichkachtinuwawon, kor miniu onau maze itinuwawon.

Inäniu au mächno echpänaneu wewon; kon oşam onau anochkihanon, kon miniu osam onau wewasehanon. Wäwänin au pemehäu waiekemazin onitchianächson miniu, asau wichkichtok au io pematesinit; kon onau tatakazichkinon, kon miniu onau pachpenozekenon. Au mächno esekeu, asau mächno kächkenohamowazin wewon onitchianächson miniu.

Wenach tä inetämoch au mächno keketotawäu waiekemazin, au anochkitawäu, au papamächtawäu miniu. Kon onau nächkosenon, au mächno keketotawäu tä, kespin nächkosinit, ene tä käz wepoz asau ani nächkosinit. Au penetahäu miniu; kon nekotoch ouau maze kikitinon, kon nekotoch onau päsikoatesenon. Maze inäniwon tä kon onau keketotawonon, kon miniu onau witchiwanon. Iachpez 
sanakat kätin, metämoch menäk iskotäu wapo. Metämoch mächno esekit kon nekotoch onau pichtikenon as tase menä kon miniu onau paketenanon wekewame weiak asau pitonik maze nepeu. Asau mazikihazin nitchionon, naskat as sikaha puanau iachpez wepoz, kon asau nepäk konomächtchiu asau sikahapuane.

Wänitchionächsitoa wawänin au kächkenohamowäwak onitchionächsowawon Anamihan, wäwänin asau anamihanit tachnäno mip nächka miniu. Au aiakoamemäwak asau ishianit as tase anamiha as mäk Käz Paketenekon tachnäno Anamihäkesekach Käz Kesekach miniu; kon tä onau paketinanowawon kotänos kaiänetoch asau otamiskanit ächpez anamiha. Wawepoz au kehomäwak onitchionächsowawon, asau aninichsinit. Iachpez weskewat as ishiach nanekot keso.

Wäwänin au kanawehäwak onıtchionächsowawon mämik echpe nächnikininit. Iachpez tä au tatepapomäwak otanowawon okisowawon miniu, kiskanitipächkat kon asau papamochnenit. Kon onau pa- 
kitinanuwawon otanowawon nachäs asau witchiwanit oske inäniwon, konopoz miniu asau keketotawanit päsikoaz esekenit. Kon onau pichtikananowawon okisowawon as tase maze esechzekä minia.

Wäwänin au anochkitawäu onitchionächsowawon, wäwänin asau anochkinit eneko äsis wichkichtonik, iachpez matät kä takazichkion. Iachpez wäwänin au aninimäu mäze esetanit au sächsewahäu, kon nekotoch asau maze esechzekenit. Kon nekotoch onau maze kikitinowawon änachnamapenit onitchionächsowawon, kon nekotoch miniu onau maze esetanowawon; iachpez kä matät ene. Au en äsehäwak miniu onitchionächsowawon kächkinohamatwone asau nenamenik masenachigon, asau kächkenamenik miniu Anamihan.

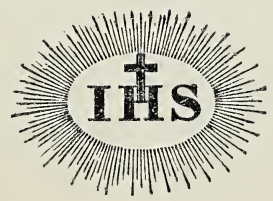


Kakichkotakan asau küchkinachkoa wäske pematesitoa, au isichziketoa, kwaiachk asau pematesitoa, ene tä asau wichkichtokoa kakik sawänechtakosin kesekoch.

Ninitchionä! An iachpez oske pemateseon, ki käz nanisanesim. Maze bawätok achpäniu ki katäu menik maze inänichtamon, maze esekin miniu. Asau wanechsemot ki tätchiak, ene kätau mächkawänechtamon, au esetaion miniu.

Tapatach penetähän iachpez wäwänin, sechkatach tä osinichtach miniu mawau päsıkoaz esekin. Käz tapanäu Hawätok paienetähänit. "Sawänechtakosewak painitähätoa, ene kä akiwak Hawätokon au näwatoa," kes hewa Jesus. Iachpez sechkanäu Hawätok päsikoatesinit, kakik iskotia tä au achpakeneu asau kotakehazin.

Asau kanawänechtach penetähän, pakichsenemin Täpänechzekät achpänin asau sawäneme asau penetähäon. Wäkiachsä isis tapasin kaiekachkoit Käz Maria, iachpez wäwänin miniu menaze- 
hen. Tachnäno nächkachkin pakichsenemin asau kanawehe äsis oweaweon, äsis otätchiakeon miniu, ene tä kätau io anamihaion nänino: "Kit anomächkon, Maria."

Wätâchnekoianin maze inänichtamon, papik wepoz, pakichsenemin Täpänechzekät asau miakonamo, kaiänit käkoch tä inänechtach. Iom tä nächkawänechtach achpäniu : ächpez mekatamon kakiz tipänichtoan, kon kinau maze esetanon; ki mächno esetom ächpez mekanat maze hawätok; kinau käz mächno echpänanik Hawätok kesekoch; as kes mekanat as kes nochkchat miniu enoch maze hawätok.

Wätächnckoianin maze inänichtamoan, ene au inänichsion: Nemewach nesik all takimakat maze onanekosin, läz wepoz au ani takimakat; wechsakänichtamon 1 ä anamachkiach kon nekotoch au ani takimakaton. Kätin ne käz wanenäwisim, kespin mänawaz onanekosin io wichkichtoaseon kakik kotakechton enis anamachkiach'.

Mächkawänechtach kaiesis wesakänechtach Jesus as kes akoze anamihäwachitiko. 
Kätin kes käz maze esckin, anoz kes nächkehäwak enoch kaies neponotoch. Iom miniu inänechtach : 'Kon ne kächkinanon echpe au nepäion. Kon konopoz nenau sawänemekonon Hawätok, konemau kon misik nenau näwanon Mächkotächkoniäu. Kespin tä anich pemateseon, as mäk käz päzeton kikäskamon, kätin. kakik iskotia nenau ishiom. Kätin anamachkiach au tanächnatäu neau kakikächkamik, kespin päsikoaz esekeon.

Manäwak kakik iskotia ächtanächnasitoa, kaies päsikoaz inänichtachkoa wächzetan,kaies nepäkoa wepoz, konomäehtchiu as näwatoa Mächkotächkoniäwon. Kätin päsikoateseu wichkoazichtoaso anamachkiach asau tanächnasit opäsik kä achpechtänechtom käkoch päsikoatach, ächpechtänemazin tä tepenau Thawätokon.

Pon nekotoch winazimon; pon nekotock pächtawon mäze kikitit; pon miniu eris tanäsenon, as tase päsikoaz kikiti; pon witchiwin päsikoatesit, näche winazimis miniu, enoch tä mäze kikitit pon keketoaswin. Enoch kätan witchiwat päsikoa- 


\section{5}

tesinit, wenach nap au päsikoaz esekeu, wenach wez pematesewon au maze tase. mik.

Pon ishianon as tase nimiheti, as tase menä miniu. Pon zenapachtach käkoch päsikoatach, nämon tä käkoch, kaiänit kotänos anapenon yapik. Pon nekotoch maze echpänanesinon, ki zenapamik Täpänechzekät, kinau käz kotakehek tä, kespin maze echpänaneseon. Iachpez matät menän; enoch täpatach iskotäu wapo au wenatesen.

Iom tä inänichsinon wawepoz: "Niniak Mäz Hawätok; kon ne katän maze esetanon änachnamapet Hawätok." .

Oske metämochsok mämikänitoa wäwänin au osimitawawak maze inäniwon waiapiskänit, kon onau witchiwanowawon, kon onau kekctotawawon. Miliächno esekit oske metämoch kon nachäs onau witchiwawon oskech inäniwon, kon onau wetapemanon as wanitipächlka kon miniu onau papamochnenon kiskanitipächka.

Katolik änamilat kon onau wenochkawanon kaiänit äs anamihanit, kon änami- 


\section{6}

hanit miniu, kon kä miniu onau wekichtinowawon. Katolik änamihat kaies wekemäu kaiänit äs anamihanit naianekotochkin kon wäwänin o wichkichtonon asan esekit, saiochkanamihat äsikit. Naianekotochkin kon o wichkichtonon asau mächno mazikihazin onitchionächson, ene tä ene kaies io enachkonikätoa Käz Mächkotächkoniäwak :

"Kon weiak Katolik änamihat onau wekemanon kaiänit äs anamihanit, konemaus miniu kon änamihanit."

Kespin katäu kanawechtawon penctähän, wawepoz paketchekenon, näskänekon nanekot keso (konopoz opäsik, kespin Mächkotächkoniäu inänechtach) otachpenach miniu Jesus Weau, kinau moskawänichtamihik tä Täpänechzekät, achpäniu asau peneteseon.

Anochkitawin wäwänin pemehin miniu kinekehekok, mämik echpe waiesakesitoawin, kätemakesitoawin, käz kitoawin miniu. Kespin weiak kozemone asau anochkitawat, ene au enat kätau anochkit: "Ne katäu ishiom anamihäwigamiko tach- 
näno Anamihäkesekachkin.” Kespin tä kon katäu paketeneon asau ishion, kon kinau anochkitonenon.

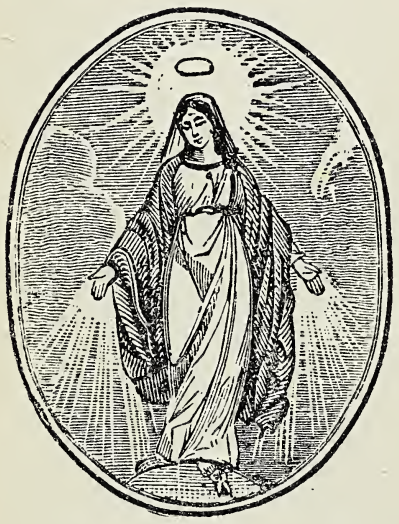


Kakichkotakan asau kächkinachkoa wänit. chionïchsitoa, au esechzeketoa, kesekoch asau ishiatoa.

Ninitchionä! Tapasin kinekehekok, menatänemin achpäniu, onamichtawin papamächtawin miniu; pon tä nekotoch kenochtamin; pon rächki keketotawin; pon nächkehin. "Kochne kikiach miniu kinau menatänemawak," kes hewa Jesus; käz Paul tä hewa: "Kinua nitchianok, papamächtokon achpäniu kinekehekowak, ene äsis mininechtach Hawätok." Iom 1ä mächkawänechtach: Saiä omos achkihe kinau mächno echpänanik Täpänechzekät, kespin mächno echpänanatoa kinekehekok, iachpez tä kinau onanikihek kesekoch. Kespin tä maze echpänanatoa, kon katäu papamächtawatoa miniu, kätin kinau tanächnasim; kinau kitämakehik omos achkihe Tapänecbzckät, kes nepäion tä kinau käz kotakehek kakik iskotia. Ene au io mächno echpänanatoa achpäniu kinekehekok, Hawätok asau sawäneme, asau mächno echpänane miniu au ächpez pemateseon achikihe. 
Ånochkitawin wäwänin kinekehekok pemänin miniu, mämik echpe waisakesitoawin, kätimakesitoawin, käz kitoawin, miniu. Pon nekotoch tapachnenanin kinekehckok; pon nekotoch miniu kikisitawin; pon maze esekenon, kinau koskänichtamihawak kinekehekok; asit tä mächno esekenon, kinau käz mininechtamihawak tä.

Pon nekotoch maze tasemin kinekchekok; kespin tä käkoch kes maze isichziketoa pon kotänos aziminon. Keketotamowin tachnäno kesekach, Mawätokon asau sawänimikotoa, asaı mächno echpä. nanekotoa, kaiesis echpänanächkoa :

Waiesakesitoawin tä kinekchokok, iachpez wäwänin kanawehanin, awe natomin tä wäwechkesit maskichkin inäniu ochtänamowin miniu waieskewacb maskichkiu, asau io inänitoa. Katiäz wesakesitoawin tä, awe natomin Mächkotächkoniäu, asau paketcheketoa kinckehekok, a-au otachpinachkoa Jesus Weau, asau otachpinachkoa miniu Anamihäu Peme, ene tä wäsetach asan isis nepäkoa, kesekoch tä asau 
ishiatoa. Kes nepäkoa tä, keketotamowin tachnäno kesekach au äz pemateseon. Asau sawänimatoa tä kinekehekok, (kaies awitoa) iachpez wawepoz akemin anamihämiguk, kotakihiswon mihikon pemochnenon, sawänemin kätimakesitoa äsis wichkichtawor, mämik tä kozemonin Mächkotächkoniäu asau paketänechsit Käz Paketenekon kinckekehekon kaies awenit io.

Mächno kcketotawin kächnäsok kächsemächsok miniu; keshiatesenon achpäniu pon maze itikon, pon miniu kichkachtikon. Menatänemin käz kitoa, pon tä nekotoch pachpenotawin. Kächkinohamatikon pos kitäshiom wäwänin asau nenamon masenachigon, anoz käkoch waieskewach asau katäu kächkenamon; achpäniu tä mächno esekenon kächkenohamatiwikamiko, papamächtawin miniu wäwänin kächkenohamo.

Tachnäro mip nächka minin anamihanon iachpez wäwänin. Kochne iachpez käshiatesit kesekoch äpit paketenamowin keall, ki tätchiakon, kitäh miniu, wech- 
tach tä: "Hawätok Nochne, ki paketäna. mon neau, ne tätchiak, netäh miniu; änänechtamon naszeau kat net äsetom iochpe as kesekach."

Tachnäno Anamihäkesekach, tachnäno Käz Kesekachkin miniu, kinau pos kit äshiom as tase anamiha iachpez änekochkitahäon wäwänin ächpez anamiha. Wawcpoz kinau nasächkawau Mächkotächkoniäu, nekotono tachnäno nekot keso otachpenach miniu Jesus Weau Käz Ostiwine. Nochpeme ächtaneseianin, kon waikichtawenin, asau näwat Mächkotächkoniäu, keke anamihanon, Käz Paketenekon zenapachtach, akemin miniu anamihämiguk anoz kakaiänit miniu anamihan.

Pon nekotoch maze kikitinon; pon nekotoch kez pemateseu nenomin maze nitchianok; pon miniu pächtawin mäze kikitit, mäze nekamit miniu. Tapasin Täpänemenach Hawätok konau tä kohsin, osimitach mawau mätä, Hawätok kinau tapanik kakikächkamik miniu kesekoch kinau sawänechtakoseket.

Käz Achpechtänechtakusit Jesus Krist! Kakikächkamik. Amen. 
Marịe käz Nikia,

Kit anomächkon;

Käz peneseon,

Käz wieskeseon.

Ki wichkichton iochpe,

Wäwänin pächta;

Asau päz nämon,

Jesus Ki Kison.

Hawätok achkano

Enachkonikäu,

Jesus Okiän,

Asau pächtaweon.

Ene tä achpäniu

Ki pakichsenemin;

Tatachkeseon

Onät Marion. Amen. 
303

\section{WAWECHTAMOWAU HAWÄTOK ASACHZEKON.}

Ne wawechtamowau Mäz Hawätok; misik tä käz Marie Kaiekachkoit, Mäz Hawätok Okiän; misik tä käz Michael, mawau käkoch kaiwaskipiskawikimakach kon asau otachpenamon.

Naszeau Jesus, Marie, Joseph, asau natamowitoa äsis wawechtamon. Amen.

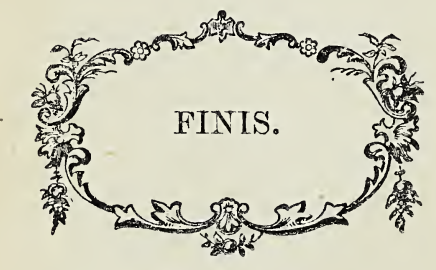





\section{Carigena.}

Page Line

13, 18, for: ohemawenit, okemawenit.

13, 22, , Kesehoch, Kesekoch.

14, 6, "katoanonwau, katoanowau.

16, 2, " ke, kes.

44, last line, for Jssus, Jesus.

45, 13, " kes, käz.

46, 13, " konomäctehiu, konomächtchiu.

47 , 8, " anänechtamon, änänechtamon.

54,7 , , mänitähäon, mänitähänon.

67, 14, ” änekochkochkitähät, änechkochkitäbät.

77, 14, " wawänechtänon, wanänechtanon.

79 , 2, ", an, au.

81, 1, " konomächtchin, konomächtchiu.

95,8 , " eue, ene.

101, 18, ", aninichsion, aninichsinın.

174, 8, , auamihawapo, anamihawapo.

185, 16, " kes koch, kesekoch.

$217,3, \quad$, äptca, äpitoa.

226,17 , " sawämematoa, sawänematoa.

228,17 , " nememach, nemewach.

230, 4, " tachmäno, tachnäno.

235, 9, ", kächkenom, kechkenon. 



\section{N D E X.}

Page.

Käz Mesachkatawän, Kakichkotakan 4 Mesachkatawäu Kesegaton ............6-8 Käz Kesegaton........................... 9

Pemochneu Kesegot..................... 11

Anamihanon ............................... 12

Nekotoasita tachnäno anino pos kächkenach, pos onamichtach änamihat $\mathbf{1 7}$ Metatach Aiakoamechnesenon ........ 18 Nekotoasita Katolik Anamihäwıgamik Ot Aiakoamechnesenon ........ 19 Nohekon Käz Ackpechtänetakosenon 20 Nohekon Käz Päzetonon .............. 20 Nekotoasita Päzetonon wä-nächkehe Waieskesit Hawätok.................. 20 Niu enon Päzetonon kesekoch äs aio ohohemakaton........................... 21 Sakäu kaianesewak o päzetonon...... 21 Nänia käz mächno esetonon........... 22 Nohekon mächno esetonon maikatımakakin mamach päzetonon........ 22 
308

Nohekon esetonon äsis otächiakit..... 23 Niu enon echpe anihimakach marnazetau o pematesin ................... 24 Shuasik Sawänechtakosenon.......... 24 . Kakichkotakan, Mip Anamihan....... 26 Mip Anamibanon...................... 27 Asäniu Ot Anamihan, Kakichkotakan 31 Mächkawänechtah, käz Marie......... 31 Ahamihan ätamä asau konomächtchiu mitchichsi......................... 32 Anamihan ätamä kaies ani mitchichsikin .................................... Nächkan Anamihan. Kakichkotakan Nächkan Anamihanon................... Anamihämiguk. Kakichkotakan..... Anamihan Michkichnon. Kakichkotakan

Anamihan wawepoz ätamä ........... 32 33 34 38 Kotakihiswon Mihikon. Kakichkotakan ....

Kotakihiswon Mihikon. Anamihan... Stabat Mater. 42 43 SIKAHAPUANITWON. Kakichkotakan Kakichkotakan os pos Sikahapuane... 44 48 48 65 70 
309

SOCHKITÄHÄSKATAWON， Kakichkotakan .......................... 73

PAKECHEKON, Kakichkotakan...... 79

Nanakatawänichswon.................. 82

" " Anamihan...... 81

Aninichswon, Kakichkotakan.......... 94

" Anamihanon ............ 98

Moskawänichtamon. Kakichkotakan 100

" " Anamihanon ... 101

Paketchekon. Kakichkotakan ........ 103

Mamiawamin Anamihanon............. 105

Miakonamatwon Sawänechzekon, Ka-

kichkotakan ........................ 107

K $\ddot{Z Z ~ E U C H A R I S T I W I N . ~ K a k i c h k o-~}$

takan ............................ 110

Käz Eucharistiwin. Anamihanon..... 120

Kes ani otachpenamächkin Jesus

Weau. Kakichkotakan

123

Anamihan ätamä kaies ani otachpena-

mächkin Käz Ostiwin............... 124

Mamiachtozekan Jesus.................. 126

KÄZ PAKETENEKON, Kakichko-

takan

131

Käz Paketenekon·Anamihanon ........ 140 
310

Kes ani Nawachkikin Anamihan. Kakichkotakan

Kez ani Nawachkikin Anamihanon... 161 nat kimänazchonau KäzOstiwin. Kakichkotakan

Kat kimänazehonau Käz Ostiwin. Anamihanon

Käz Otäh Jesus. Kakichkotakan...... 178

66

"Anamihanon 180

ANAMIHÄU PEME. Kakichkotakan 182 66 6 Anamihanon.... 209

Kakichkotakan Waiesakesitoa io...... Anamihanon 66 “...... 194

Kakichkotakan asau kächkinachkoa au isichziketoa akiko känawihatoa 200 Käz Niman. Kakichkotakon.. 202 66 66 Anamihanon 204

Kakichkotakan katäu anich pematesitoa io. 212

Anamibanon katäu anich pematesitoa io 214

Mamiachtozekan katäu anich peinatesitoa io............................... 217

Kakichkolakan wäwänin asau sawänematoa anino kaies nepäkoa....... 226 
Nekamon echpe Mächkotächkoniäu as paketänemazin näpikoa............... 230 Libera, Dies Irae, Miserere. 230,235 Anamihan ätamä echpe pakitänemechzin mäz aia. 237 Anamihan echpe päkitänemạchzin nitchion 238 MÄCHKOTÄCHKONIÄWIN. Kakichkotakan ........................... 240 Nekoneneu Kwaiachkichne Anamihan ................................. 250 ANA.IIHÄU WIKICHTWON....... 268 Kakichkotakan, asau kächkinackoa waikichtitoa au isichzikitoa.......... 287 Wäske Pematesitoa. Kakichkotakan 292 Nitchionosok. Kakichkotakaı........ 298 Mani käz Nikia. Nekamon............. 802 Wawechtamowau Hawätok Asachzekon ..................................... 303 


\title{
ALPHABETICAL INDEX.
}

\begin{abstract}
Page.
Act of Contrition .....................16, 28 Acts of Faith, Hope, Charity........16, 28 Amendment, Firm purpose of......... 101 " Instruction on ........... 100 Angel Guardian, Prayer to......29, 99, 199 Angelus ................................. 14

" Instruction on................... 31
\end{abstract} Asperges ................................. 141 Baptism, Instruction on ............. 65 " Private, Instruction on ...... 70 Beatitudes ............................... 24 Beuediction, Prayers at.............160, 171 Blessed Eucharist, Instruction on...... 110 Blessed Sacrament, Instruction on visiting the ........................ 173 Blessed Sacrament, Prayers at visiting 175 Burials, Instructions for...... ........ 226 “ Prayers at ..................... 237 
313

Calendar for Lent...................... 6 " for Vigils and Ember days... 7, 8 " for Ascension and Corpus

Xti

Catholic Religion, only true ........... 250

Children, Instructions for the........... 298 Commandements of God................ 18

"6 of the Church ....... 19

Communion, Instruction on........110, 123 "6

Prayers before and

after................120, 124

Confesion, Instruction on............... 103

“ Prayers before and after 8I, 105

Confirmation, Instruction on ........... 73

Confiteor ................................. 15

Contrition, Instruction on .............. 94

" Acts of....................16, 98

Creed, The Apostles' .................. 13

Cross, Sign of the ..................... 12

" Way of the, Instruction on...... 44

" Way of the, Prayers ............ 48

Dead, Instruction on praying for the 226

"Menominee hymns sung for the 230

" Prayers for the................... 237 
314

Dies Irae, In Menominee................ 232

Dying, Instruction for the..... ........ 212

" Prayers for the................... 214

" Litany for the.................. 217

Eucharist, Instruction on the Bl...... 110

Examination of Consience............. 82 66

Prayers before and

atter ................ 81, 98

Extreme Unction, Justruction on...... 182

" " Prayers before and

atter...........................209, 210

Fasting, Instruction on................ 4

Glory be to the Father, etc............. 13

Grace before and after meals........... 32

Guardian Angel, Prayer 10.... .29, 99, 199

Hail Mary............................... 12

Holy Days................................. 9

Holy Spirit, Prayers to .............76, 77

" "Seven Gifts of the.... . 73

" "Six sins against the ..... 20

Holy Orders, Instruction............... 240

Hymns, In Menominee...167, 1i2, 216, 302

Heart of Jesus, Instruction on......... 178

" i6 " Prayers to.............. 180 
315

I Believe in God.......................... 13

Indulgences, Instruction on ............ 107 Instruction on Amendment ............. 100

\begin{tabular}{|c|c|}
\hline & " Angelus .................... \\
\hline 66 & “ Baptism ............ \\
\hline 66 & Private.......... \\
\hline " & "the Blessed Eucharist \\
\hline 66 & "Visiting the Bl, Sacr... \\
\hline "6 & " Burials ............ \\
\hline " & " the Catholic Religion \\
\hline 6 & " Holy Communion...110 \\
\hline “ & " Confession................. \\
\hline “ & " Confirmation ............. \\
\hline “ & " Contrition ................ \\
\hline " & " the Way of the Cross \\
\hline " & " Praying for the Dead \\
\hline « & for the Dying ............. \\
\hline “ & on Extreme Unction .. \\
\hline " & “ Fasting ................ \\
\hline “ & “ Holy Orders............ \\
\hline “ & " Indulgences .............. \\
\hline “ & " Holy Mass ............. \\
\hline “ & " Matrimony ................ \\
\hline & " Morning Prayers....... \\
\hline
\end{tabular}


316

Instruction on Night Prayers......... 33

" $\quad$ " $\quad$ " Penance, Sacrament of 79

" . " the Rosary B. V.M.... 38

" for Married People, Parents..................... 287

" for Young Pectple.......... 292

" for Children................. 298

" on the Sacred Heart of Jesus ................ 178

" on the Scapular........... 42

" for the Sick................ 185

" for their Nurses............ 200

" on the Viaticum.......... 20\%

" on Vespers.................. 160

Jesus, Litary of the Holy Name of... 126 Joseph, Prayers to St..............9, 212 Lent, Instruction for................... 4

Letters, Pronunciation of.............. 3

Libera. In Menominee.................. 230

Litany of the Holy Name of Jesus ... 126

Litany for the Dying ................ 217

Magnificat. In Menominee.......... . 170

Mary Virgin, Prayers to.....12, 15, 31, 36,

$43,99,181,197,209,211$ 
317

Mass, Instruction Holy.................. 131

Mass, Prayers at Holy.................. 140

Matrimony, Instruction on ........... 268

Married People, Instruction for........ 287

Memorare. In Menominee ............... 31

Michael Arch. Prayers to............ . 30, 70

Miserere. In Menominee ................ 235

Morning Prayers....................... 27

" " Instruction on ....... 26

Night Prayers............................ 34

" " Instruction on........... 33

Our Father....... ........................ 12

O Salutaris. In Menominee............. 172

Patron Saint, Prayer to the...........30, 70 Penance. Instruction on the Sacrament.................................. 79

Pledge of Total Abstinence............ 303

Private Baptism, Instruction on...... 70

Priesthood, Instruction................. 240

Pronunciation of Letters.............. 3

Purgatory, Instruction on...........186, 228

Recommendation of the Dying........ 220

Rosary, Instruction on the.............. 38 Rules of Life for the Different

States...................... 287, 292-98 
318

Sacraments, the Seven................. 20

Sacred Heart of Jesus, Instruction.... 178

" " " Prayers to the 180

Scapular, Instruction on the .......... 42

" Prayers for Those Wearing

the ...........................

43

Sick, Instruction for the................ 185

" Prayers of the ..................... 194

"Instruction for the Nurses of the 200

Sins, Seven deadly....................... 20

"Six against the Holy Ghost ...... 20

" Four crying to Heaven............ 21

" Nine accessory..................... 21

Stabat Mater. ............................. 48

Stations, Instructions on the........... 44

6. Prayers at the................. 48

Things, The four last of man........... 24

Vespers, Instruction on ................ 160

" Psalms in Menominee........ 161

Viaticum, Instruction on the.......... 202

" Prayers on and after receiving.. 
Virtues, Seven opposed to the deadly sins

Works, The seven corporal............ 2s

" The seven spiritual............ 23

" Three good...................... 22

Young People, Instruction for........ 292

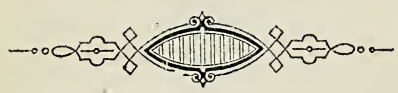









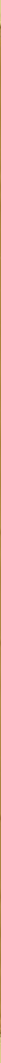


(5) 